\title{
The Minimum Principle of SINR: A Useful Discretization Tool for Wireless Communication
}

\author{
EREZ KANTOR, MIT, USA \\ ZVI LOTKER, Ben Gurion University, Israel \\ MERAV PARTER and DAVID PELEG, Weizmann Institute of Science, Israel
}

\begin{abstract}
Theoretical study of optimization problems in wireless communication often deals with tasks that concern a single point. For example, the power control problem requires computing a power assignment guaranteeing that each transmitting station $s_{i}$ is successfully received at a single receiver point $r_{i}$. This paper aims at addressing communication applications that require handling two-dimensional tasks (e.g., guaranteeing successful transmission in entire regions rather than at specific points).

The natural approach to two-dimensional optimization tasks is to discretize the optimization domain, e.g., by sampling points within the domain. The straightforward implementation of the discretization approach, however, might incur high time and memory requirements, and moreover, it cannot guarantee exact solutions.

The alternative proposed and explored in this paper is based on establishing the minimum principle ${ }^{1}$ for the signal to interference and noise ratio (SINR) function with free space path loss (i.e., when the signal decays in proportion to the square of the distance between the transmitter and receiver). Essentially, the minimum principle allows us to reduce the dimension of the optimization domain without losing anything in the accuracy or quality of the solution. More specifically, when the two-dimensional optimization domain is bounded and free from any interfering station, the minimum principle implies that it is sufficient to optimize the SINR function over the boundary of the domain, as the "hardest" points to be satisfied reside on the boundary and not in the interior.

We then utilize the minimum principle as the basis for an improved discretization technique for solving two-dimensional problems in the SINR model. This approach is shown to be useful for handling optimization problems over two dimensions (e.g., power control, energy minimization); in providing tight bounds on the number of null cells in the reception map; and in approximating geometric and topological properties of the wireless reception map (e.g., maximum inscribed sphere).
\end{abstract}

${ }^{1} \mathrm{~A}$ function satisfies the minimum principle if its minimum in any compact domain is attained at the domain's boundary.

A preliminary version of this paper appeared in IEEE 56th Annual Symposium on Foundations of Computer Science, FOCS 2015, Berkeley, CA, USA, 17-20 October, 2015.

Zvi Lotker is partially supported by Foundation des Sciences Mathematiques de Paris, the Ministry of Science Technology and Space, Israel, French-Israeli project MAIMONIDE 31768XL, and the French-Israeli Laboratory FILOFOCS. Erez Kantor is supported in a part by AFOSR Contract Numbers : FA9550-13-1-0042 and FA9550-14-1-0403, and by NSF Awards 0939370-CCF, CCF-1217506 and CCF-AF-0937274. David Peleg and Merav Parter are supported in part by the Israel Science Foundation (grant 894/09), the United States-Israel Binational Science Foundation (grant 2008348), the Israel Ministry of Science and Technology (infrastructures grant), and the Citi Foundation.

Authors' addresses: E. Kantor, MIT, Cambridge, USA; email: erezk@csail.mit.edu; Z. Lotker, Ben Gurion University, Beer Sheva, Israel; email: zvilo@cse.bgu.ac.il; M. Parter and D. Peleg, Weizmann Institute of Science, Rehovot, Israel; emails: \{merav.parter, david.peleg\}@weizmann.ac.il.

Permission to make digital or hard copies of all or part of this work for personal or classroom use is granted without fee provided that copies are not made or distributed for profit or commercial advantage and that copies bear this notice and the full citation on the first page. Copyrights for components of this work owned by others than ACM must be honored. Abstracting with credit is permitted. To copy otherwise, or republish, to post on servers or to redistribute to lists, requires prior specific permission and/or a fee. Request permissions from permissions@acm.org.

(c) 2023 Association for Computing Machinery.

1549-6325/2023/02-ART1 \$15.00

https://doi.org/10.1145/3477144 
The minimum principle, as well as the interplay between continuous and discrete analysis presented in this paper, are expected to pave the way to future study of algorithmic SINR in higher dimensions.

CCS Concepts: • Theory of computation $\rightarrow$ Data structures design and analysis; Network optimization;

Additional Key Words and Phrases: SINR, communication, power control

ACM Reference format:

Erez Kantor, Zvi Lotker, Merav Parter, and David Peleg. 2023. The Minimum Principle of SINR: A Useful Discretization Tool for Wireless Communication. ACM Trans. Algor. 19, 1, Article 1 (February 2023 ), 45 pages. https://doi.org/10.1145/3477144

\section{INTRODUCTION}

\subsection{Background and Motivation}

This paper concerns the fundamental goal of developing useful discretization tools for optimization problems in wireless networks. We study wireless communication in free space [8]. This idealized model is simpler than the irregular environment of radio channels in a general setting, which involves reflection and shadowing. We consider the Signal to Interference-plus-Noise Ratio (SINR) model, where given a set of stations $S=\left\{s_{1}, \ldots, s_{n}\right\}$ in $\mathbb{R}^{d}$ concurrently transmitting with power assignment $\psi$, and ambient (sometimes referred to as background) noise $N$, a receiver located at point $p \in \mathbb{R}^{d}$ successfully receives a message from station $s_{i}$ if and only if $\operatorname{SINR}\left(s_{i}, p\right) \geq \beta$, where

$$
\operatorname{SINR}\left(s_{i}, p\right)=\frac{\psi_{i} \cdot \operatorname{dist}\left(s_{i}, p\right)^{-\alpha}}{\sum_{j \neq i} \psi_{j} \cdot \operatorname{dist}\left(s_{j}, p\right)^{-\alpha}+N}
$$

for constants $\alpha$ (denoting the path-loss exponent) and $\beta>0$ (denoting the reception threshold, namely, the minimum SINR required for a message to be successfully received), and where dist() denotes Euclidean distance. Throughout, we assume $\alpha=2$, which is the path-loss exponent commonly assumed to apply in free space (cf. [8]). The SINR model for $\alpha=2$, as all other physical models for wireless networks, yields reception zones that are continuous in space (except at transmitter points) and can be defined by a characteristic polynomial $\widetilde{H}(p)$ of degree $\Theta(n)$ (defined at the beginning of Section 2, and more explicitly for $d=2$ in Section 4, Equation (13)).

In practical network optimization tasks, it is usually insufficient to achieve a desired property at a single target point; rather, it is required that a certain (two-dimensional) region satisfies a desired property (e,g., successful transmission reception by a given station). The observation motivating our work is that optimization over two dimensional space is rather complicated when dealing with high degree characteristic polynomials as arise by the SINR function. Previous theoretical work in this area avoided this difficulty by focusing on a single reception point. For example, in the power control problem, ${ }^{2}$ one is given $n$ communication links $\left\langle s_{i}, r_{i}\right\rangle$ where $r_{i}$ is point, and a target SINR threshold $\beta$ and the goal is to compute a feasible transmission power assignment $\psi$ with respect to $\beta$, that is, a power assignment that achieves $\operatorname{SINR}\left(s_{i}, r_{i}\right) \geq \beta$ for every $i \in\{1, \ldots, n\}$, where all stations transmit according to $\psi$. In other words, the problem is restricted to simple requirements, where each station $s_{i}$ has to be received at a single point $r_{i}$. In contrast, in the current paper we aim at studying more general problems in the SINR model, namely, problems pertaining to entire reception regions rather than single reception points.

The first natural approach to such generalization is to discretize the two-dimensional optimization domain, e.g., by sampling many points in the given two-dimensional region. This brute-force

\footnotetext{
${ }^{2}$ For a comprehensive review on the power control problem, see [6].
} 
approach has two main shortcomings. From a quantitative point of view, the resulting time complexity depends upon the area of the optimization domain and hence the size of the new program might be very large. From a qualitative point of view, a priori this approach is doomed to be an approximation scheme and can never result in an exact solution, even in cases where an exact solution can be obtained in polynomial time for the 0 -dimensional case. The uncertainty for unsampled points can be decreased by increasing the sampling resolution, but it can never be completely avoided.

The current paper provides an attractive alternative to the above brute-force approach, by focusing on a basic analytic tool known as the minimum principle. Specifically, we establish the minimum principle of the SINR function in free space and demonstrate its power as a useful discretization technique. Generally, a function satisfies the minimum principle if its minimum in any compact domain is attained at the domain's boundary. The minimum principle (dually known as the maximum principle) has been widely studied (in a number of variations with somewhat different assumptions) and is one of the useful tools employed in studying partial differential equations [13], most notably for elliptic, parabolic, and hyperbolic PDE's. We show that the minimum principle of the SINR function has several algorithmic applications. In particular, we establish its usefulness in optimization problems over two dimensions (e.g., power control); in providing tight bounds on the number of null cells; and in approximating geometric and topological properties of the wireless reception map (e.g., maximum inscribed sphere) faster than before. The power of the minimum principle is that it reduces the dimension of the optimization domain without losing anything in the accuracy or quality of the solution. More specifically, as long as the two-dimensional optimization domain is bounded and free from any interfering station, the minimum principle implies that it is sufficient to optimize over the boundary of the domain, as the "hardest" points to be satisfied reside on the boundary of the domain and not in its interior. Such one-dimensional optimization is significantly more tractable than optimization in two dimensions, as the time complexity is no longer scaled with the area of the optimization region but rather with its perimeter.

An additional benefit of this approach is that in certain cases, it can yield an exact solution, in the following sense. Consider a reception testing problem where one is given a wireless network, a target station $s_{i}$, a desired reception threshold $\beta$, and a closed polygon $\mathcal{P}$, defined by rational vertices and free from interfering stations, i.e., $\mathcal{P} \cap\left(S \backslash\left\{s_{i}\right\}\right)=\emptyset$. The task is to decide if the entire area of $\mathcal{P}$ is receptive to the transmission of the station $s_{i}$, i.e., $\operatorname{SINR}\left(s_{i}, p\right) \geq \beta$, for every $p \in \mathcal{P}$. Without relying on the minimum principle, a plausible straightforward way to approach this is to sample sufficiently many points within $\mathcal{P}$ and to evaluate the SINR value at each such point. Since there is no guarantee that the unsampled points are receptive, this scheme cannot decide in finite time if $\mathcal{P}$ is receptive. It follows that the answer we obtain for such points is only approximate, in the sense that it guarantees an SINR value above $\beta-\epsilon$ for some $\epsilon$. In contrast, the minimum principle allows us to provide the guarantee that the SINR value over the entire region is at least $\beta$. Specifically, by exploiting properties of rational univariate polynomials, one can decide in polynomial time if every edge $\sigma$ of $\mathcal{P}$ is receptive in its entirety or not. In particular, in contrast to the two-dimensional input polygon $\mathcal{P}$, the polygon edge $\sigma$ is a line segment (of dimension 1), and thus testing reception on it is more tractable. The minimum principle then implies that $\mathcal{P}$ is receptive iff every edge of it is receptive.

We hope that these new discretization tools will encourage the future study of two-dimensional optimization problems in the SINR model. In particular, we believe that these tools should aid us in handling the generalization of the joint scheduling and power control problem from a single point to a two-dimensional domain. The complexity of this problem (for a single point) in the physical model, taking into account the geometry of the problem, is not fully understood. Nevertheless, many algorithms and heuristics have been suggested for it, e.g., [5, 7, 9, 15-17, 23, 24]. 


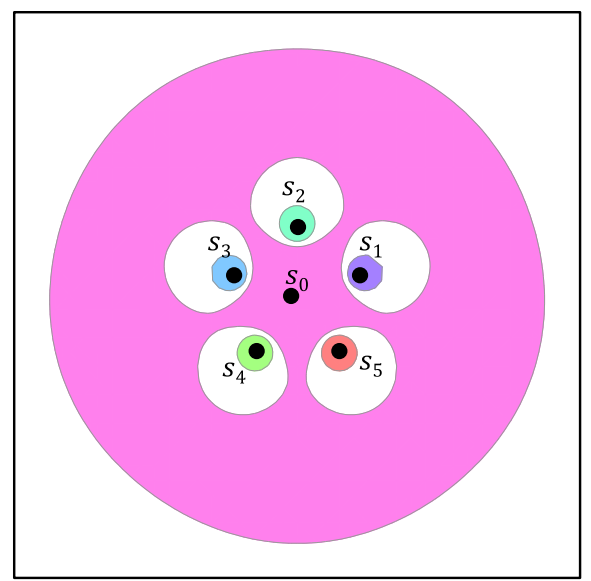

Fig. 1. Illustration of an $n$-station network with $n-1$ null cells (depicted for $n=6$ ). A strong station $s_{0}$ is placed at the center of a circle and $n-1$ weak stations $s_{1}, \ldots, s_{n-1}$ are positioned on the circle.

From the topological point of view, the minimum principle also allows us to give a better topological characterization of the wireless communication map. To model the reception regions we use the convenient representation of an SINR diagram, introduced in [3], which partitions the plane into $n$ reception zones, one per station, and the complementary region where no station's signal can be received. The topology and geometry of SINR diagrams were studied in [3] in the relatively simple setting of uniform power, where all stations transmit with the same power level. SINR diagrams under the general non-uniform setting (i.e., with arbitrary power assignments) were studied in [10]. The topological features of general SINR diagrams turned out to be more complicated than in the uniform case. Several important properties of SINR diagrams were established in [10]. One of the key results demonstrates that when the system is embedded in $d$-dimensional space $\mathbb{R}^{d}$, the reception regions in $\mathbb{R}^{d+1}$ (i.e., one dimension higher than that in which the stations are embedded) are hyperbolically convex. Hence, although the $d$-dimensional map might be highly fractured, drawing the map in one dimension higher makes the zones (hyperbolically) connected. So far, the challenge of establishing useful properties that hold in the dimension $d$ where the network is embedded remains open. It was conjectured in [10] that certain undesirable configurations are in fact excluded in (general) $d$-dimensional SINR diagrams. In particular, there are no holes in a reception region that is free of interfering stations. In other words, every hole in a reception cell must contain at least one interfering station. This property, termed "No-Free-Hole" in [10], is defined as follows. A collection $C$ of compact domains in $\mathbb{R}^{d}$ obeys the No-Free-Hole property with respect to a station set $S$ if for every domain $C \in C$ that is free of stations, if all the boundary points of $C$ are reception points of $s_{i}$, then all the (internal) points of $C$ are reception points of $s_{i}$ as well. In [10] (see also [12]), the No-Free-Hole property was established only for the reception regions of 1-dimensional networks in free space (i.e., with a path-loss exponent $\alpha=2$ ) and was conjectured to hold for any dimension. By showing that the SINR function satisfies the minimum principle, the No-Free-Hole conjecture is resolved for every dimension $d \geq 1$ and for SINR threshold $\beta>0$. Consequently, every null cell (or hole) "surrounded" by a reception region of station $s_{i}$ (formally meaning that it is contained in the outer Jordan curve bounding some reception cell of $s_{i}$ ) must contain some interfering station $s_{j}$. See Figure 1 . 


\subsection{Contributions}

The main technical contribution of this paper involves establishing the minimum principle for the SINR function in free space (i.e., for path-loss exponent $\alpha=2$ ). Our approach involves a characterization of the "hard" network configurations for which establishing the minimum principle requires a more subtle analysis. An essential step in our analysis is providing a closed and elegant form for the average strength of a station on the boundary of a $d$-dimensional ball, which may be of independent interest. Extending the result to other $\alpha$ values remains a challenging open problem.

We then present several applications of the minimum principle, briefly reviewed next. Mainly, we demonstrate the applicability of the minimum principle as a discretization tool that enables us to handle standard two-dimensional problems in the SINR model. Some of the presented applications (e.g., polygonal power control, universal linear bound on the number of null cells) are technically nontrivial and call for new tools. Others (e.g., computing the maximum inscribed sphere) are mostly built upon existing tools but may be of significant practical interest.

Resolving the No-Free-Hole conjecture. In Section 3 we improve our topological understanding of SINR maps by resolving the No-Free-Hole conjecture raised in [10]. The minimum principle implies that although the reception regions are not convex in general, they enjoy a certain type of convexity (or smoothness) in station-free regions.

Exact and approximate schemes of reception testing. The first algorithmic application that illustrates the usefulness of our result concerns the setting of reception testing, introduced in Section 4.2. The input for this problem is a wireless network, a compact domain $C$ and a target station $s_{0}$. It is required to decide if $C$ is fully receptive for the station $s_{0}$, namely, if it is fully contained in the reception region of $s_{0}$. We provide two alternative reception testing schemes. First, for the case where the input shape $C$ is a polygon whose vertices are positioned at rational coordinates in the plane, we provide (in Section 4.3) an exact reception testing scheme that returns in polynomial time "yes" iff $C$ is receptive for $s_{0}$. Then, for the general case of any compact domain $C$, we present (in Section 4.4) an approximate reception testing procedure that by evaluating the SINR function for the points on the boundary of $C$ can make deductions regarding the reception quality of the entire domain $C$.

Maximum inscribed sphere inside a reception region. In Section 4.5 we consider the following problem. Given an $n$-station network and a target station $s_{i}$, compute the maximum sphere around $s_{i}$ that is fully contained in the reception region of $s_{i}$. We show that using the minimum principle, and in particular the approximate reception testing scheme of Section 4.4, one can compute an approximation for this problem in improved time compared to what could have been done using the basic approach without the minimum principle.

The polygonal power control problem. In Section 4.6 we define the following problem, which is a two-dimensional generalization of the well-known power control problem. Given $n$ stations $s_{1}, \ldots, s_{n}$ along with $n$ polygons $\mathcal{P}_{1}, \ldots, \mathcal{P}_{n}$ and a desired SINR threshold $\beta$, find a transmission power assignment $\psi$ such that the SINR value of every reception point $p \in \mathcal{P}_{i}$ with respect to station $s_{i}$ is at least $\beta$ when all stations transmit simultaneously according to $\psi$. We show that this problem corresponds to a convex program and present a separation oracle that can be used as a black box by the Ellipsoid algorithm for solving this problem. ${ }^{3}$ The same scheme applies also for the sum-power minimization problem and the min-max power problem, in which it is also required to minimize the total (respectively, maximum) transmission power [6].

\footnotetext{
${ }^{3}$ We remark that the problem is solvable more generally on any closed region whose boundary is composed of arcs representable in parametric form by a univariate constant-degree polynomial.
} 
A universal bound of the number of null cells. In Section 4.7 we consider the theoretical challenge of providing a tight bound for the number of null cells in the reception map for SINR threshold $\beta>$ 1. Note that in the presence of ambient noise there is only one unbounded null cell (see Lemma 4.17), and hence the number of bounded null cells equals the number of null cells minus one. In [10] it is shown, using Milnor-Thom Theorem, that there are $O\left(n^{2 d}\right)$ null cells for every dimension $d \geq 1$. In this paper, we tighten this into linear bound of $O(n)$ on the number of null cells for every dimension (which is tight up to constants). Our proof strategy combines a topological and continuous characterization of the system on the one hand, along with a discrete analysis of the graph representation induced by the collection of null cells.

Approximation of the number of null cells. The upper bound of $O(n)$ on the number of null cells established in Section 4.7 holds for every network. Nevertheless, for certain networks the actual number of null cells can be considerably lower, which can be useful for algorithmic purposes. Consequently, in Section 4.8 we provide an approximation scheme ${ }^{4}$ for the number of null cells surrounded by a given reception region. This approximation is obtained by exploiting the fact that the minimum principle also implies a lower bound on the area of the null cells (i.e., the null cells cannot be arbitrarily small) and in addition, it implies that every null cell contains an interfering station. These observations lead to an efficient approximate null cell detection scheme: every null cell in the SINR map is detected and every detected null cell exists in an SINR map of slightly smaller SINR threshold.

\section{PRELIMINARIES}

Geometric notions: We consider the $d$-dimensional Euclidean space $\mathbb{R}^{d}$ (for $d \in \mathbb{Z}_{\geq 1}$ ). The distance between points $p$ and $q$ is denoted by $\operatorname{dist}(p, q)=\|q-p\|$. A ball of radius $r$ centered at point $p \in \mathbb{R}^{d}$ is the set of all points at distance at most $r$ from $p$, denoted by $B^{d}(p, r)=\left\{q \in \mathbb{R}^{d} \mid\right.$ $\operatorname{dist}(p, q) \leq r\}$. The basic notions of open, closed, bounded, compact and connected sets of points are defined in the standard manner. The closure of a point set $P$, denoted $\operatorname{cl}(P)$, is the smallest closed set containing $P$ (i.e., the set consisting of all points in $P$ together with all limit points of $P)$. The boundary of $P$ is the intersection of the closure of $P$ and the closure of its complement, i.e., $\operatorname{bd}(P)=\operatorname{cl}(P) \cap \operatorname{cl}(\bar{P})$. A maximal connected subset $P_{1} \subseteq P$ is a connected point set such that $P_{1} \cup\{p\}$ is no longer connected for every $p \in P \backslash P_{1}$. A domain $D$ in Euclidean space is an open connected set. We use the term zone to describe a point set with some "niceness" properties. Unless stated otherwise, a zone refers to the union of an open connected set and some subset of its boundary. Let $\widetilde{H}: \mathbb{R}^{d} \rightarrow \mathbb{R}$ be a polynomial and let $p \in \mathbb{R}^{d}$. Then $\widetilde{H}$ is the characteristic polynomial of a zone $Z$ if $p \in Z \Leftrightarrow \widetilde{H}(p) \leq 0$. For a non-empty bounded zone $Z$ and an interior point $p$ of $Z$, denote the maximal and minimal radii of $Z$ with respect to $p$ by $\delta(p, Z)=\sup \{r>0 \mid Z \supseteq B(p, r)\}$ and $\Delta(p, Z)=\inf \{r>0 \mid Z \subseteq B(p, r)\}$.

Wireless networks: We consider a wireless network $\mathcal{A}=\langle d, S, \psi, N, \beta, \alpha\rangle$, where $d \in \mathbb{Z}_{\geq 1}$ is the space dimension, $S=\left\{s_{0}, s_{1}, \ldots, s_{n}\right\}$ is a set of transmitting radio stations embedded in $d$ dimensional space, $\psi$ is an assignment of a positive real transmission power $\psi_{i}$ to each station $s_{i}$, $N \geq 0$ is the ambient (or background) noise, $\beta \geq 0$ is a constant reception threshold (to be explained soon), and $\alpha \geq 1$ is the path-loss parameter. The network is assumed to contain at least two stations, i.e., $n \geq 1$. The strength of station $s_{i}$ at point $p \neq s_{i}$ is defined as $\mathrm{E}_{\mathcal{A}}\left(s_{i}, p\right)=\psi_{i} \cdot \operatorname{dist}\left(s_{i}, p\right)^{-\alpha}$. The signal to interference \& noise ratio (SINR) of $s_{i}$ at point $p$ is defined as

$$
\operatorname{SINR}_{\mathcal{A}}\left(s_{i}, p\right)=\frac{\mathrm{E}_{\mathcal{A}}\left(s_{i}, p\right)}{\sum_{s_{j} \in\left(S \backslash\left\{s_{i}\right\}\right)} \mathrm{E}_{\mathcal{A}}\left(s_{i}, p\right)+N}=\frac{\psi_{i} \cdot \operatorname{dist}\left(s_{i}, p\right)^{-\alpha}}{\sum_{j \neq i} \psi_{j} \cdot \operatorname{dist}\left(s_{j}, p\right)^{-\alpha}+N} .
$$

\footnotetext{
${ }^{4}$ The nature and quality of the approximation are explained therein.
} 
Observe that $\operatorname{SINR}_{\mathcal{A}}\left(s_{i}, p\right)$ is positive by definition. In certain contexts, it may be more convenient to consider the reciprocal of the SINR function,

$$
\operatorname{SINR}_{\mathcal{A}}^{-1}\left(s_{i}, p\right)=\frac{1}{\psi_{i}}\left(\sum_{j \neq i} \psi_{j}\left(\frac{\operatorname{dist}\left(s_{i}, p\right)}{\operatorname{dist}\left(s_{j}, p\right)}\right)^{\alpha}+N \cdot \operatorname{dist}\left(s_{i}, p\right)^{\alpha}\right) .
$$

When the network $\mathcal{A}$ is clear from the context, we may omit it and write simply $\mathrm{E}\left(s_{i}, p\right)$ and $\operatorname{SINR}\left(s_{i}, p\right)$. The fundamental rule of the SINR model is that the transmission of station $s_{i}$ is received correctly at point $p \notin S$ if and only if its signal to noise ratio at $p$ is no smaller than the reception threshold of the network, i.e., the reception condition is

$$
\operatorname{SINR}\left(s_{i}, p\right) \geq \beta .
$$

In this case, we say that the signal of $s_{i}$ is received at $p$. We refer to the set of points that receive the signal of station $s_{i}$ as the reception zone of $s_{i}$, defined as

$$
\mathcal{H}_{i}(\mathcal{A})=\left\{p \in \mathbb{R}^{d}-S \mid \operatorname{SINR}_{\mathcal{A}}\left(s_{i}, p\right) \geq \beta\right\} \cup\left\{s_{i}\right\} .
$$

This definition is necessary since $\operatorname{SINR}\left(s_{i}, \cdot\right)$ is undefined at points in $S$ and in particular at $s_{i}$ itself. Note that $\mathcal{H}_{i}(\mathcal{A})$ is not necessarily connected. A connected component (i.e., a maximal connected subset of points) within a zone is referred to as a cell. In the same manner we refer to the set of points that do not receive a signal from any station of $S$ (due to the background noise and interference) as the null zone. For technical reasons we define the null zone formally using nonstrict inequalities, namely, as

$$
\mathcal{H}_{\emptyset}(\mathcal{A})=\left\{p \in \mathbb{R}^{d}-S \mid \operatorname{SINR}\left(s_{i}, p\right) \leq \beta \text { for every } s_{i} \in S\right\} .
$$

Note that when $\beta>1$, for any point $p$, at most one of the inequalities of the reception zones $\mathcal{H}_{i}(\mathcal{A})$ can become an equality. The points at which this happens are precisely the boundary points of the reception zones. Hence our definition implies that the boundary points belong also to the null zone. Also note that the null zone is not necessarily connected. In general, $\mathcal{H}_{\emptyset}(\mathcal{A})$ is composed of $\tau_{\emptyset}(\mathcal{A})$ connected cells $\mathcal{H}_{\emptyset, j}(\mathcal{A})$, i.e., $\mathcal{H}_{\emptyset}(\mathcal{A})=\bigcup_{j=1}^{\tau_{\emptyset}(\mathcal{A})} \mathcal{H}_{\emptyset, j}(\mathcal{A})$.

An SINR diagram $\mathcal{H}(\mathcal{A})=\left\{\mathcal{H}_{i}(\mathcal{A}) \mid 0 \leq i \leq n\right\} \cup\left\{\mathcal{H}_{\emptyset}(\mathcal{A})\right\}$ is a "reception map" characterizing the reception zones of the stations. For $\beta>1$, this map partitions ${ }^{5}$ the plane into $n+2$ zones; a reception zone $\mathcal{H}_{i}(\mathcal{A})$ for each station $s_{i}, 0 \leq i \leq n$, and a null zone $\mathcal{H}_{\emptyset}(\mathcal{A})$ where no transmissions are received successfully.

The following important technical lemma from [3] is useful in our later analysis.

LEMMA 2.1 ([3]). Let $f: \mathbb{R}^{d} \rightarrow \mathbb{R}^{d}$ be a mapping consisting of rotation, translation, and scaling by a factor of $\sigma>0$. Consider some network $\mathcal{A}=\langle d, S, \psi, N, \beta, \alpha\rangle$ and let $f(\mathcal{A})=\left\langle d, f(S), \psi, N / \sigma^{2}, \beta, \alpha\right\rangle$, where $f(S)=\left\{f\left(s_{i}\right) \mid s_{i} \in S\right\}$. Then $f$ preserves the signal to noise ratio, namely, $\operatorname{SINR}_{\mathcal{A}}\left(s_{i}, p\right)=$ $\operatorname{SINR}_{f(\mathcal{A})}\left(f\left(s_{i}\right), f(p)\right)$ for every station $s_{i}$ and point $p \notin S$.

The Minimum Principle. A function $f$ satisfies the minimum principle (a.k.a. the weak minimum principle) if for every compact domain $D$ such that $f$ is continuous on every point in $D$, the minimum of $f$ on $D$ is attained on the boundary $\operatorname{bd}(D)$ of the domain, i.e., $f(p) \geq \min \left\{f\left(p^{\prime}\right) \mid p^{\prime} \in\right.$ $\operatorname{bd}(D)\}$ for every $p \in D$. If the minimum is attained only on the boundary, i.e., the above inequality holds with a strict inequality, then the function satisfies the strong minimum principle. The strong and weak maximum principles are defined analogously. (We remark that several slightly different definitions of the minimum principle appear in the literature, depending on the context. Here, we

\footnotetext{
${ }^{5}$ We slightly abuse terminology by ignoring overlaps with the null zone at the boundaries.
} 
adopted the definition most suitable for the SINR setting, rather than trying to capture the most general conditions in which the principle holds.)

\section{THE STRONG MINIMUM PRINCIPLE OF THE SINR FUNCTION}

Consider an $n+1$ station network $\mathcal{A}=\langle d, S, \psi, N, \beta, \alpha=2\rangle$. The strong minimum principle for the $\operatorname{SINR}_{\mathcal{A}}$ function says that for every compact domain $D \subset \mathbb{R}^{d}$ that is free from stations ${ }^{6}$ of $S$, $\operatorname{SINR}_{\mathcal{A}}\left(s_{i}, p\right)>\min \left\{\operatorname{SINR}_{\mathcal{A}}\left(s_{i}, p^{\prime}\right) \mid p^{\prime} \in \operatorname{bd}(D)\right\}$, for every interior point $p$ of $D$.

The assumption that $D \cap S=\emptyset$ is necessary to ensure that $\operatorname{SINR}_{\mathcal{A}}\left(s_{i}, p\right)$ is continuous on $D$. Our main result is the following.

TheOREm 3.1. For every network $\mathcal{A}=\langle d, S, \psi, N, \beta, \alpha=2\rangle$, the function $\operatorname{SINR}_{\mathcal{A}}$ satisfies the strong minimum principle.

Towards proving this, we first show a more restricted result, namely, that the strong minimum principle is satisfied in the special case where the domain $D$ is a station-free $d$-dimensional ball.

Proposition 3.2. For every $n+1$ station network $\mathcal{A}=\langle d, S, \psi, N, \beta, \alpha=2\rangle$, the function $\operatorname{SINR}_{\mathcal{A}}$ satisfies the strong minimum principle on any station-free ball.

To see that Proposition 3.2 implies Theorem 3.1, suppose towards contradiction that the strong minimum principle does not hold on some compact domain $D$ that is free of stations. Then if $p$ is an interior point of $D$ achieving the minimum SINR value over all of the point of $D$, then there must be some sufficiently small ball $B$ centered at $p$ such that $p$ attains the minimum value over all points in $B$. This is then in contradiction with Proposition 3.2.

The remainder of this section is dedicated to proving Proposition 3.2 and subsequently Theorem 3.1. Specifically, Section 3.1 introduces the basic notions of continuous average strength and collinear rotation and provides a number of useful claims for later use. Section 3.2 presents two sufficient conditions for the minimum principle of SINR on a ball to hold. Armed with these tools, Section 3.3 presents a high-level overview and some intuition for the proof structure. Section 3.4 establishes Proposition 3.2 (i.e., proves the minimum principle on a ball).

\subsection{Some Useful Tools}

Throughout this section, we use the following conventions. For simplicity, we first illustrate some of the main ideas on the two-dimensional case $d=2$ (our formal proofs apply to the general case of $d \geq 2$ ). We focus on station $s_{0}$ and show that the function $\operatorname{SINR}_{\mathcal{A}}\left(s_{0}, p\right)$ satisfies the minimum principle. Consider a point $q \in \mathbb{R}^{2}$ such that $q$ is not equidistant from all of the stations. (This assumption too is eliminated later on.) Consider a real $r>0$ such that all stations are outside the ball $B(q, r)$. That is, there exists a station $s_{j} \in S$ such that $\operatorname{dist}\left(s_{0}, q\right) \neq \operatorname{dist}\left(s_{j}, q\right)$; and $s_{i} \notin B(q, r)$ for every $s_{i} \in S$. Hereafter, let $\mathrm{B}=B(q, r)$ and let $\rho_{i}=\operatorname{dist}^{2}\left(s_{i}, q\right)$ for every $i=0, \ldots, n$. By Lemma 2.1 , let us assume without loss of generality that the radius of the ball is $r=1$ and its center is at $q=(0,0)$, and that $s_{0}$ is located on the positive $y$-axis (i.e., $\mathrm{B}=B((0,0), 1)$ and $s_{0}=\left(0, \sqrt{\rho_{0}}\right)$ ).

As we show later on, it would be sufficient to show that the SINR value at the center of the ball $q$ is larger than the minimum SINR on the boundary of the ball. Towards that goal, we compare the SINR value at $q$ to the "average" SINR value on the boundary of the ball the value. In addition, we focus our attention on the four intersection points of the ball's boundary bd(B) with the $x$ and $y$ axes, namely,

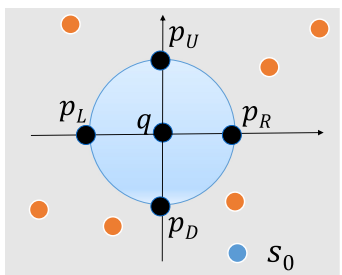

\footnotetext{
${ }^{6}$ The added restriction that the domain is free from stations is needed in order to make sure that the SINR function is defined on every point in the domain.
} 
$p_{L}=(-1,0), p_{R}=(1,0), p_{D}=(0,-1)$ and $p_{U}=(0,1)$ (see figure).

Our proof will show that either the SINR value at the center point is (strictly) larger than the SINR value attained at these four points, or else it is larger than the "average" SINR value on the boundary.

For technical reasons, instead of making our arguments on the SINR function, we work instead on the reciprocal of the SINR function, and show that it satisfies the maximum principle. We next formalize a notion that measures the average behavior of the reciprocal SINR function on the boundary of the ball.

Continuous average strength. Our analysis makes use of the notion of continuous average strength for balls [11]. The average strength of $s_{i}$ experienced at the boundary bd(B) of the ball $\mathrm{B}=\mathrm{B}(q, r)$ is given by

$$
\varepsilon_{i}(q, r)=\frac{1}{2 \pi r} \int_{p \in \operatorname{bd}(\mathrm{B})} \mathrm{E}_{\mathcal{A}}\left(s_{i}, p\right) .
$$

In Appendix B, we generalize this measure to any $d \geq 2$ and show the following.

LEMMA 3.3. $\varepsilon_{i}(q, r) \geq \psi_{i} /\left|\operatorname{dist}\left(s_{i}, q\right)^{2}-r^{2}\right|$.

(Let us remark that extending this lemma from $\alpha=2$ to general $\alpha$ is the main barrier to extending the minimum principle to any $\alpha$.)

Denote the average signal to average interference ratio on the ball's boundary bd(B) by

$$
\operatorname{AVG}\left(\mathcal{A}, s_{i}\right)=\frac{\varepsilon_{i}(q, r)}{\sum_{j \neq i} \varepsilon_{j}(q, r)+N} .
$$

Denote the minimum value of $\operatorname{SINR}_{\mathcal{A}}\left(s_{i}, p\right)$ on the boundary bd(B) by $\operatorname{MIN}\left(\mathcal{A}, s_{i}\right)$, and the corresponding maximum value of $\operatorname{SINR}_{\mathcal{A}}^{-1}\left(s_{i}, p\right)$ on $\operatorname{bd}(\mathrm{B})$ by $\operatorname{MAX}\left(\mathcal{A}, s_{0}\right)$. That is,

$$
\operatorname{MIN}\left(\mathcal{A}, s_{i}\right)=\min \left\{\operatorname{SINR}_{\mathcal{A}}\left(s_{i}, p\right) \mid p \in \operatorname{bd}(\mathrm{B})\right\}
$$

and

In addition, let

$$
\operatorname{MAX}\left(\mathcal{A}, s_{0}\right)=\max \left\{\operatorname{SINR}_{\mathcal{A}}^{-1}\left(s_{i}, p\right) \mid p \in \operatorname{bd}(\mathrm{B})\right\}=\frac{1}{\operatorname{MIN}\left(\mathcal{A}, s_{0}\right)} .
$$

$$
\operatorname{MAX}\left(\mathcal{A}, s_{0}, p_{L}, p_{R}\right)=\max \left\{\operatorname{SINR}_{\mathcal{A}}^{-1}\left(s_{0}, p_{L}\right), \operatorname{SINR}_{\mathcal{A}}^{-1}\left(s_{0}, p_{R}\right)\right\}
$$

The following claims, proven in Appendix C.1, are useful later in our analysis.

LEMmA 3.4. (a) $\operatorname{MAX}\left(\mathcal{A}, s_{0}\right) \geq \operatorname{AVG}^{-1}\left(\mathcal{A}, s_{0}\right)$ with equality if and only if $\operatorname{SINR}_{\mathcal{A}}\left(s_{0}, p\right)=\operatorname{AVG}\left(\mathcal{A}, s_{0}\right)$ for every $p \in \operatorname{bd}(\mathrm{B})$.

(b) $\operatorname{MAX}\left(\mathcal{A}, s_{0}\right) \geq \operatorname{MAX}\left(\mathcal{A}, s_{0}, p_{L}, p_{R}\right)$.

Collinear rotations. The notion of collinear rotations is easiest to describe for $d=2$. Consider a noise-free wireless network $\mathcal{A}=\langle d=2, S, \psi, N=0, \beta \geq 1, \alpha\rangle$, where $S=\left\{s_{0}, s_{1} \ldots, s_{n}\right\}$. Denote the origin point by $q=(0,0)$, let $p_{R}=(1,0), p_{L}=(-1,0)$ and define $\rho_{i}=\operatorname{dist}^{2}\left(s_{i}, q\right)$, for every $i=0, \ldots, n$. It is convenient to concentrate on the simple case where all interfering stations are aligned on the $y$-axis, namely, the line between $s_{0}$ and the ball center $q$. Formally, the network $\mathcal{A}$ is called $y$-collinear if all stations are aligned on the $y$-axis. (For a higher dimension $d>2$, collinearity requires that all stations are aligned on a common $(d-1)$-dimensional hyperplane.) In our setting, this guarantees the important property that $\operatorname{dist}\left(s_{i}, p_{L}\right)=\operatorname{dist}\left(s_{i}, p_{R}\right)$ for every $s_{i} \in S$. For a network $\mathcal{A}$ in which the stations are in general position, it is convenient to consider a modification of $\mathcal{A}$ into a $y$-collinear network with the same SINR value for $s_{0}$. This modified network, referred to hereafter as the collinear rotation of $\mathcal{A}$ and denoted $C R(\mathcal{A})$, is obtained from $\mathcal{A}$ by rotating the 


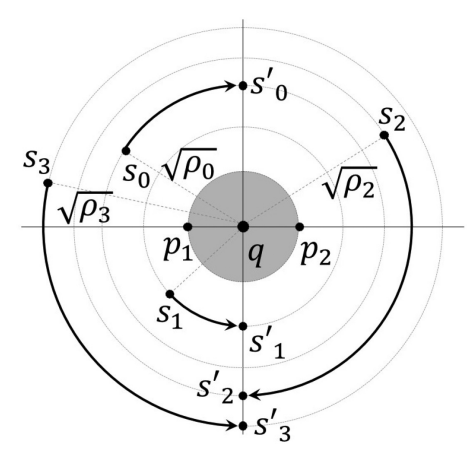

Fig. 2. The collinear rotation network $C R(\mathcal{A})$. The interfering stations $s_{i} \neq s_{0}$ are relocated to the negative $y$-axis while the target station $s_{0}$ is mapped to the positive $y$-axis.

stations around the ball center $q$. More specifically, given $\mathcal{A}=\langle d=2, S, \bar{\psi}, N=0, \beta \geq 1, \alpha=2\rangle$, the collinear rotation of $\mathcal{A}$,

$$
C R(\mathcal{A})=\left\langle S^{\prime}=\left\{s_{0}^{\prime}, \ldots, s_{n}^{\prime}\right\}, \bar{\psi}, N=0, \beta=1, \alpha\right\rangle,
$$

is obtained from $\mathcal{A}$ as follows. Rotate the station $s_{0}$ around the origin point $q$, maintaining its distance from $q$, until it reaches the point $\left(0, \sqrt{\rho_{0}}\right)$ on the positive $y$-axis. Similarly, rotate each of the stations $s_{1}, \ldots, s_{n}$ around the origin $q$, maintaining its distance from $q$, until it reaches the point $\left(0,-\sqrt{\rho_{i}}\right)$ on the negative $y$-axis. The stations $\left.s_{0}^{\prime}, s_{1}^{\prime}, \ldots, s_{n}^{\prime}\right)$ are therefore collinear on the $y$ axis and preserve the distances of $s_{0}, \ldots, s_{n}$, respectively, from $q$. This ensures that an interfering station $s_{i}^{\prime}$ is never co-located with the target station $s_{0}^{\prime}$, even when $\rho_{i}=\rho_{0}$. The construction is illustrated in Figure 2 (where $\left.\rho_{1}=\rho_{0}\right)$. Moreover, note that $\operatorname{SINR}_{\mathcal{A}}\left(s_{0}, q\right)=\operatorname{SINR}_{\mathcal{A}^{\prime}}\left(s_{0}^{\prime}, q\right)$.

The following lemma is essential for the main results of this paper. It is a generalization of Proposition 3.6 of [3] for a wireless system with $n+1$ stations. It holds for any network $\mathcal{A}$ and for any dimension $d$, but the proof (presented in appendix C.2) goes more easily by considering first the case where $\mathcal{A}$ has dimension $d=2$, and establishing the proof for the collinear rotation network $C R(\mathcal{A})$.

Lemma 3.5. Let $\mathcal{A}$ be a noise-free network $(N=0)$ and let $q \notin S$. Then

$$
\max \left\{\operatorname{SINR}_{\mathcal{A}}^{-1}\left(s_{0}, p_{L}\right), \operatorname{SINR}_{\mathcal{A}}^{-1}\left(s_{0}, p_{R}\right)\right\} \geq \sum_{i=1}^{n} \frac{\psi_{i}}{\psi_{0}} \cdot\left(\frac{\rho_{0}+1}{\rho_{i}+1}\right)^{\alpha / 2},
$$

with equality only if $\operatorname{dist}\left(s_{i}, p_{L}\right)=\operatorname{dist}\left(s_{i}, p_{R}\right)$ for every $s_{i} \in S \backslash\left\{s_{0}\right\}$.

The following measure plays a key role in our analysis. This measure coincides with the rightside measure in Lemma 3.5 when $N=0$. Hence, it can be thought of as the extension for the case where there is noise in the network.

$$
\operatorname{SUM}\left(\mathcal{A}, s_{0}\right)=\sum_{i=1}^{n} \frac{\psi_{i} \cdot\left(\rho_{0}+1\right)}{\psi_{0} \cdot\left(\rho_{i}+1\right)}+\frac{N \cdot\left(\rho_{0}+1\right)}{\psi_{0}} .
$$

Our analysis takes special care of $y$-collinear networks. As will be shown later, for such networks a tighter characterization (or bounds) can be obtained.

\subsection{Sufficient Conditions for the Minimum Principle of SINR on a Ball}

In this subsection we propose two sufficient conditions for establishing the strong minimum principle of the SINR function on the ball. 
The ball center condition. Given an $n+1$ station network $\mathcal{A}=\langle d, S, \psi, N, \beta, \alpha=2\rangle$, the $\operatorname{SINR}_{\mathcal{A}}$ function is said to satisfy the ball center condition if for every station $s_{i} \in S$,

$$
\operatorname{SINR}_{\mathcal{A}}\left(s_{i}, q\right)>\min \left\{\operatorname{SINR}_{\mathcal{A}}\left(s_{i}, p\right) \mid p \in \operatorname{bd}(B(q, r))\right\},
$$

for every station-free ball $B(q, r)$ such that the ball center $q$ is not equidistant from all stations. We now show that the ball center condition is sufficient for establishing Proposition 3.2. (The proof is in Appendix C.3.)

Lemma 3.6. If the $n+1$ station network $\mathcal{A}$ satisfies the ball center condition, then Proposition 3.2 holds, namely, the SINR function satisfies the strong minimum principle for every station-free ball.

We next provide the second sufficient condition.

The dual minimality condition. The $\operatorname{SINR}_{\mathcal{A}}$ function satisfies the dual minimality condition on the ball $\mathrm{B}$ if and only if one of the following two conditions holds:

$$
\operatorname{SINR}_{\mathcal{A}}^{-1}\left(s_{0}, q\right)<\operatorname{MAX}\left(\mathcal{A}, s_{0}, p_{L}, p_{R}\right)
$$

or

$$
\operatorname{SINR}_{\mathcal{A}}^{-1}\left(s_{0}, q\right)<\operatorname{AVG}^{-1}\left(\mathcal{A}, s_{0}\right) .
$$

Alternatively, these conditions can be written in compact form as follows:

$$
\operatorname{SINR}_{\mathcal{A}}^{-1}\left(s_{0}, q\right)<\max \left\{\operatorname{MAX}\left(\mathcal{A}, s_{0}, p_{L}, p_{R}\right), \operatorname{AVG}^{-1}\left(\mathcal{A}, s_{0}\right)\right\} .
$$

The function $\operatorname{SINR}_{\mathcal{A}}$ satisfies the weak dual minimality condition on B if Equation (6) holds with a non-strict inequality.

By Lemmas 3.6 and 3.4, we get the following.

LEMMA 3.7. The dual minimality condition is sufficient for satisfying the ball center condition and hence also the strong minimum principle for the ball.

By Lemma 3.6, in order to prove Proposition 3.2 it suffices to restrict attention to $s_{0} \in S$ and the ball $\mathrm{B}$, and show that the $\operatorname{SINR}_{\mathcal{A}}$ function satisfies the ball center condition, namely, $\operatorname{SINR}_{\mathcal{A}}\left(s_{0}, q\right)>$ $\operatorname{MIN}\left(\mathcal{A}, s_{0}\right)$.

\subsection{Proof Overview}

Before delving into the detailed proof of the minimum principle for the SINR function, let us first outline the proof structure of Proposition 3.2 at a high level. We first establish the strong ball center condition for the noise-free case. One of the key insights, in this context, is that the expressions $\operatorname{SINR}_{\mathcal{A}}\left(s_{0}, q\right), \operatorname{AVG}^{-1}\left(\mathcal{A}, s_{0}\right)$ and $\operatorname{SUM}\left(\mathcal{A}, s_{0}\right)$ are all functions of the transmission powers $\psi_{0}, \ldots, \psi_{n}$ and the distances $\rho_{0}, \ldots, \rho_{n}$ between $q$ and the $s_{i}$ 's. Moreover, as will be shown, for the $y$-collinear case, $\operatorname{MAX}\left(\mathcal{A}, s_{0}, p_{L}, p_{R}\right)=\operatorname{SUM}\left(\mathcal{A}, s_{0}\right)$. To establish the minimum principle, we begin by partitioning the station set $S$ into three subsets according to their distance $\rho_{i}$ from $q$ compared to the distance $\rho_{0}$,

$$
S_{\text {close }}=\left\{s_{i} \in S \mid \rho_{i}<\rho_{0}\right\}, S_{e q}=\left\{s_{i} \in S \mid \rho_{i}=\rho_{0}\right\} \text { and } S_{\text {far }}=\left\{s_{i} \in S \mid \rho_{i}>\rho_{0}\right\} .
$$

To gain some intuition, consider two extreme cases. For a network $\mathcal{A}$ all of whose stations are far $\left(S=S_{\text {far }}\right)$, Equation (4) holds, hence the dual minimality condition is satisfied. On the other hand, for a network $\mathcal{A}$ all of whose stations are close $\left(S=S_{\text {close }}\right)$, Equation (5) holds, hence again the dual minimality condition is satisfied. The general noise-free case (where $\mathcal{A}$ consists of both far and close stations) is also based on this intuition, but is more involved, and is based on showing, by applying some algebraic manipulations, that if $\operatorname{SINR}_{\mathcal{A}}^{-1}\left(s_{0}, q\right) \geq \operatorname{AVG}^{-1}\left(\mathcal{A}, s_{0}\right)$ then $\operatorname{SINR}_{\mathcal{A}}^{-1}\left(s_{0}, q\right)<\operatorname{MAX}\left(\mathcal{A}, s_{0}, p_{L}, p_{R}\right)$, as required. 
We then turn to consider the general noisy case. To prove the weak version of the ball center condition, we use an infinite family $\mathcal{N F}$ of a noise-free networks that behave similarly to the noisy network at the points of the ball $\mathrm{B}$. The reasoning is by contradiction, showing that the assumption that the weak version of the ball center condition does not hold, implies the existence of a noisefree network in the family $\mathcal{N} \mathcal{F}$ that violates the strong ball center condition (which was already established for the noise-free case). Turning to the strong version of the ball center condition, the analysis considers two cases of network configurations. The first case is of $y$-collinear networks. In this case, the analysis exploits the fact that the 1-dimensional SINR function satisfies the minimum principle (note that the dimension of $y$-collinear networks is in fact one). Finally, we consider the remaining case where the network is not $y$-collinear and yet Ineq. (6) holds with equality. The reasoning for this case proceeds along the following steps. We first claim that $\operatorname{SINR}_{\mathcal{A}}^{-1}\left(s_{0}, q\right)=$ $\operatorname{AVG}^{-1}\left(\mathcal{A}, s_{0}\right)$. This is shown again by using the infinite family $\mathcal{N} \mathcal{F}$. We complete the main part of the proof by showing that $\operatorname{AVG}^{-1}\left(\mathcal{A}, s_{0}\right)>\operatorname{MAX}\left(\mathcal{A}, s_{0}, p_{L}, p_{R}\right)$. To do that, two auxiliary noisefree networks are constructed. The first is $\mathcal{A}^{\prime}=C R(\mathcal{A})$, obtained from $\mathcal{A}$ by collinear rotation. The second network, $\mathcal{A}^{\prime \prime}$, is obtained from $\mathcal{A}^{\prime}$ by a slight and careful perturbation to the location of the main station. By using the tight bounds previously achieved for $y$-collinear networks, the desired claim is established.

\subsection{Proof of the Minimum Principle on a Ball}

We first consider the simpler case where there is no ambient noise (i.e., $N=0$ ) and show that the SINR function satisfies the dual minimality condition and hence also the strong minimum principle. (The proof is deferred to Appendix C.4.)

LEMmA 3.8. Let $\mathcal{A}_{0}=\langle d, S, \psi, N=0, \beta, \alpha=2\rangle$ be an $n+1$ station network with no ambient noise. Then the $\operatorname{SINR}_{\mathcal{A}_{0}}$ function satisfies the dual minimality condition (and hence also the strong minimum principle).

We now turn to consider the more general case where the network $\mathcal{A}$ has a positive ambient noise $N>0$. The reasoning for this case is more involved and consists of the following stages. In Section 3.4.1, we first prove that the $\operatorname{SINR}_{\mathcal{A}}$ function satisfies the weak minimum principle. The following two subsections establish the strong minimum principle for the $\operatorname{SINR}_{\mathcal{A}}$ function, where Section 3.4.2 considers the simpler case of $y$-collinear networks, and Section 3.4.3 deals with networks in general position.

3.4.1 The Weak Minimum Principle on a Ball for Noisy Networks. We begin by establishing the weak version of the minimum principle for the noisy setting. Specifically, we show that the $\operatorname{SINR}_{\mathcal{A}}$ function satisfies the dual minimality condition in the weak sense, i.e., it satisfies Equation (6) with a non-strict inequality.

Mimicking $\mathcal{A}$ by an infinite sequence of noise-free networks. Our proof technique makes use of an infinite family of noise-free networks $\mathcal{A}_{\ell}$, for $\ell \in \mathbb{R}_{>1}$, that mimic the noisy network $\mathcal{A}$ at the points of the ball $\mathrm{B}$ as $\ell$ gets sufficiently large. Let

$$
\mathcal{N F}=\{\mathcal{A}(\ell) \mid \ell>1\},
$$

where each network $\mathcal{A}(\ell)=\left\langle d, S_{\ell}, \bar{\psi}_{\ell}, N=0, \beta, \alpha\right\rangle$ consists of $n+2$ stations, $S_{\ell}=S \cup\left\{s_{\ell}\right\}$, namely, the $n+1$ stations of $\mathcal{A}$ plus an additional station, $s_{\ell}=(0, \ell)$, whose position and transmission power are parameterized by $\ell$. Intuitively, $\mathcal{A}(\ell)$ is obtained from $\mathcal{A}$ by replacing the ambient noise $N$ with an additional station $s_{\ell}$ that plays a similar role to that of the noise at the points of the ball B as $\ell$ tend to infinity. The "noise-simulating" station $s_{\ell}$ is located at the point $(0, \ell)$ and its transmission power is $\psi_{\ell}=N \cdot \ell^{2}$, hence the received signal strength of $s_{\ell}$ at point $q$ equals 
that of the noise $N$. The other transmission powers remain unchanged, so $\bar{\psi}_{\ell}=\left(\psi_{0}, \ldots, \psi_{n}, \psi_{\ell}\right)$, and hence

$$
\mathrm{E}_{\mathcal{A}(\ell)}\left(s_{\ell}, q\right)=\psi_{\ell} \cdot \operatorname{dist}\left(s_{\ell}, q\right)^{-2}=N .
$$

Lemma 3.9. The following properties hold for every $\mathcal{A}_{\ell} \in \mathcal{N} \mathcal{F}$.

(P1) $\operatorname{SINR}_{\mathcal{A}(\ell)}^{-1}\left(s_{0}, q\right)=\operatorname{SINR}_{\mathcal{A}}^{-1}\left(s_{0}, q\right)$;

(P2) $\lim _{\ell \rightarrow \infty} \mathrm{AVG}^{-1}\left(\mathcal{A}(\ell), s_{0}\right)=\mathrm{AVG}^{-1}\left(\mathcal{A}, s_{0}\right)$;

(P3) $\lim _{\ell \rightarrow \infty} \operatorname{MAX}\left(\mathcal{A}(\ell), s_{0}, p_{L}, p_{R}\right)=\operatorname{MAX}\left(\mathcal{A}, s_{0}, p_{L}, p_{R}\right)$;

(P4) $\operatorname{MAX}\left(\mathcal{A}(\ell), s_{0}, p_{L}, p_{R}\right)<\operatorname{MAX}\left(\mathcal{A}, s_{0}, p_{L}, p_{R}\right)$.

Proof. (P1) holds by Equation (8). Recall that $\mathrm{E}_{\mathcal{A}(\ell)}\left(s_{\ell}, p\right)=N \cdot \ell^{2} / \operatorname{dist}\left(s_{\ell}, p\right)^{2}$ for every $p \in$ $\mathrm{bd}(\mathrm{B})$, and by the triangle inequality, $\ell-1 \leq \operatorname{dist}\left(s_{\ell}, p\right) \leq \ell+1$. Hence,

$$
N \cdot\left(\frac{\ell}{\ell+1}\right)^{2} \leq \mathrm{E}_{\mathcal{A}}\left(s_{\ell}, p\right) \leq N \cdot\left(\frac{\ell}{\ell-1}\right)^{2},
$$

and thus $\lim _{\ell \rightarrow \infty} \mathrm{E}_{\mathcal{A}(\ell)}\left(s_{\ell}, p\right)=N$ for every $p \in \operatorname{bd}(\mathrm{B})$. Properties (P2) and (P3) follow. It remains to consider (P4). Since $\operatorname{dist}\left(s_{\ell}, p_{i}\right)^{2}=\ell^{2}+1$ for $i \in\{L, R\}$, the received signal strength of $s_{\ell}$ at point $p_{i}$ is $\mathrm{E}_{\mathcal{A}(\ell)}\left(s_{\ell}, p_{i}\right)=N \cdot \frac{\ell^{2}}{\ell^{2}+1}<N$. Hence $\operatorname{SINR}_{\mathcal{A}(\ell)}\left(s_{0}, p_{i}\right)>\operatorname{SINR}_{\mathcal{A}}\left(s_{0}, p_{i}\right) \operatorname{or}_{\operatorname{SINR}}^{-1}\left(s_{\mathcal{A}(\ell)}\left(s_{0}, p_{i}\right)<\right.$ $\operatorname{SINR}_{\mathcal{A}}^{-1}\left(s_{0}, p_{i}\right)$ for $i \in\{L, R\}$. Property (P4) follows.

We now show that when $N \geq 0$, the minimum principle holds in the weak sense (i.e., with a non-strict inequality).

LEMmA 3.10. For $N \geq 0$, the $\operatorname{SINR}_{\mathcal{A}}$ satisfies the weak dual minimality condition, i.e.,

$$
\operatorname{SINR}_{\mathcal{A}}^{-1}\left(s_{0}, q\right) \leq \max \left\{\operatorname{MAX}\left(\mathcal{A}, s_{0}, p_{L}, p_{R}\right), \operatorname{AVG}^{-1}\left(\mathcal{A}, s_{0}\right)\right\} .
$$

Proof. Suppose, towards contradiction, that

$$
\operatorname{SINR}_{\mathcal{A}}^{-1}\left(s_{0}, q\right)>\operatorname{AVG}^{-1}\left(\mathcal{A}, s_{0}\right) \text { and } \operatorname{SINR}_{\mathcal{A}}^{-1}\left(s_{0}, q\right)>\operatorname{MAX}\left(\mathcal{A}, s_{0}, p_{L}, p_{R}\right) .
$$

This implies, together with properties (P2) and (P3) of Lemma 3.9, that there exists a sufficiently large number $\ell^{*}$ such that $\mathcal{A}\left(\ell^{*}\right) \in \mathcal{N} \mathcal{F}$ and

$$
\operatorname{SINR}_{\mathcal{A}}^{-1}\left(s_{0}, q\right)>\operatorname{AVG}^{-1}\left(\mathcal{A}\left(\ell^{*}\right), s_{0}\right) \text { and } \operatorname{SINR}_{\mathcal{A}}^{-1}\left(s_{0}, q\right)>\operatorname{MAX}\left(\mathcal{A}\left(\ell^{*}\right), s_{0}, p_{L}, p_{R}\right) .
$$

Combining this with property (P1) of Lemma 3.9, we get that

$$
\operatorname{SINR}_{\mathcal{A}\left(\ell^{*}\right)}^{-1}\left(s_{0}, q\right)>\operatorname{AVG}^{-1}\left(\mathcal{A}\left(\ell^{*}\right), s_{0}\right) \text { and } \operatorname{SINR}_{\mathcal{A}\left(\ell^{*}\right)}^{-1}\left(s_{0}, q\right)>\operatorname{MAX}\left(\mathcal{A}\left(\ell^{*}\right), s_{0}, p_{L}, p_{R}\right) \text {. }
$$

Since $\mathcal{A}\left(\ell^{*}\right)$ is a noise-free network, this is in contradiction with Lemma 3.8 (taking $\mathcal{A}_{0}=\mathcal{A}\left(\ell^{*}\right)$ ). The lemma follows.

3.4.2 The Strong Minimum Principle on a Ball for Noisy Collinear Networks $(d=2)$. In this subsection, we establish the strong minimum principle for $y$-collinear networks (where all stations are aligned on the $y$-axis). The analysis of this case exploits the weak minimum principle for 1dimensional networks that was established in $[10,12]$.

Fact 3.11 (WeAk Minimum Principle in $\mathbb{R}^{1}[10,12]$ ). In a 1-dimensional network (i.e., where all stations are aligned on a line), the SINR function satisfies the weak minimum principle.

As explained in [10], Fact 3.11 immediately implies the following corollary (adapted to the current setting where the stations of the 1 -dimensional network are embedded in $\mathbb{R}^{2}$ on the $y$-axis instead on the $x$-axis). 
Corollary 3.12 (Weak Minimum Principle for Collinear Networks in $\mathbb{R}^{2}$ ). Consider a network $\mathcal{A}$ where each $s_{i} \in S$ is positioned on $\left(0, y_{i}\right)$. Let $q_{1}$ and $q_{2}$ be two points on the $y$-axis, such that there is no station of $S$ on the segment $\overline{q_{1} q_{2}}$. Then for every $p \in \overline{q_{1} q_{2}}$

$$
\operatorname{SiNR}_{\mathcal{A}}\left(s_{0}, p\right) \geq \operatorname{MIN}\left(\mathcal{A}, s_{0}, q_{1}, q_{2}\right) .
$$

We next claim, however, that the minimum principle holds also in the strong sense for collinear networks. (The proof appears in Appendix C.5.)

Lemma 3.13 (Strong Minimum Principle for Collinear Networks in $\mathbb{R}^{2}$ ). Consider a network $\mathcal{A}$ where each $s_{i} \in S$ is positioned on $\left(0, y_{i}\right)$. Let $q_{1}$ and $q_{2}$ be two points on the $y$-axis, such that there is no station of $S$ on the segment $\overline{q_{1} q_{2}}$. Then for every $p \in \overline{q_{1} q_{2}}$

$$
\operatorname{SINR}_{\mathcal{A}}\left(s_{0}, p\right)>\operatorname{MIN}\left(\mathcal{A}, s_{0}, q_{1}, q_{2}\right) .
$$

Lemma 3.14. Let $\mathcal{A}$ be a $y$-collinear network. Then $\operatorname{SINR}_{\mathcal{A}}$ satisfies the strong minimum principle on the (station-free) ball $\mathrm{B}$.

Proof. Recall that $p_{D}=(0,-1)$ and $p_{U}=(0,1)$ are the two points on the intersection of the $y$-axis and $\operatorname{bd}(\mathrm{B})$, hence $\operatorname{MAX}\left(\mathcal{A}, s_{0}, p_{D}, p_{U}\right) \geq \operatorname{MAX}\left(\mathcal{A}, s_{0}\right)$. Since $\overline{p_{D} p_{U}} \subset \mathrm{B}$ and $\mathrm{B}$ is free from stations, by the strong minimum principle for the collinear case (Lemma 3.13), $\operatorname{SINR}_{\mathcal{A}}^{-1}\left(s_{0}, q\right)<$ $\operatorname{MAX}\left(\mathcal{A}, s_{0}, p_{D}, p_{U}\right)$. Combining the two inequalities, we get that the ball center condition (Equation (3)) holds. The claim now follows by Lemma 3.6.

3.4.3 The Strong Minimum Principle on a Ball for Noisy Non-Collinear Networks (any d). In this section, we consider a non-collinear network $\mathcal{A}$ not all of whose stations are aligned on the $y$-axis. We have shown before (in Lemma 3.10) that such a network satisfies the weak dual minimality condition. We now consider two subcases. If $\mathcal{A}$ also satisfies the strong dual minimality condition, then we are done by Lemma 3.7. Hence, it remains to consider the case where

$$
\operatorname{SINR}_{\mathcal{A}}^{-1}\left(s_{0}, q\right)=\max \left\{\operatorname{MAX}\left(\mathcal{A}, s_{0}, p_{L}, p_{R}\right), \operatorname{AVG}^{-1}\left(\mathcal{A}, s_{0}\right)\right\}
$$

Indeed, we believe that this can be realized by some networks, namely, there exist networks that do not satisfy the dual minimality condition. Yet, as will be shown now, the strong minimum principle still holds.

We first establish this for the two-dimensional setting. To do that, we begin by showing (in Claim C.2) that if Equality (11) holds then the SINR value at the center point $q$ is equal to the average SINR value on the ball's boundary, i.e., $\operatorname{SINR}_{\mathcal{A}}^{-1}\left(s_{0}, q\right)=\operatorname{AVG}^{-1}\left(\mathcal{A}, s_{0}\right)$. To prove this, we exploit properties (P1), (P2) and (P4) of Lemma 3.9 to show that in this special case, the dominating parameter is the average $\mathrm{SINR}^{-1}$ value on the ball's boundary, namely,

$$
\operatorname{AVG}^{-1}\left(\mathcal{A}, s_{0}\right) \geq \operatorname{MAX}\left(\mathcal{A}, s_{0}, p_{L}, p_{R}\right) .
$$

By Inequality (12) there are now only two cases to consider. The first is where $\operatorname{AVG}^{-1}\left(\mathcal{A}, s_{0}\right)=$ $\operatorname{MAX}\left(\mathcal{A}, s_{0}, p_{L}, p_{R}\right)$. We show that this case can be attained only for $y$-collinear networks. Hence it remains to consider the complementary case, where $\operatorname{AVG}^{-1}\left(\mathcal{A}, s_{0}\right)>\operatorname{MAX}\left(\mathcal{A}, s_{0}, p_{L}, p_{R}\right)$. Claim C.3 shows that under this setting, the strong minimum principle is guaranteed to hold. In summary, we obtain the following lemma, establishing the strong minimum principle for twodimensional non-collinear networks. (The detailed proof of this lemma appears in Appendix C.6).

Lemma 3.15. For $d=2$, if $\mathcal{A}$ is a non-collinear network, then $\operatorname{SINR}_{\mathcal{A}}$ satisfies the strong minimum principle on any station-free ball. 
Proof of Proposition 3.2. We prove the claim by induction on the dimension $d$. For the induction base, $d=1$, the property holds by Lemma 3.13 .

Next, assume that this property holds for any $d-1 \geq 1$ and consider dimension $d$. Note that as long as $\operatorname{MAX}\left(\mathcal{A}, s_{0}, p_{L}, p_{R}\right)>\operatorname{SUM}\left(\mathcal{A}, s_{0}\right)$ is satisfied, the proof follows the exact same line as for collinear networks. The only extreme case that it remains to consider is where $\operatorname{MAX}\left(\mathcal{A}, s_{0}, p_{L}, p_{R}\right)=\operatorname{SUM}\left(\mathcal{A}, s_{0}\right)$. By Claim C.1(a), in $\mathbb{R}^{2}$, this happens iff the network is $y$ collinear. Hence the only adaptation required for general dimension $d>2$ is the extension of the definition of $y$-collinear networks to higher dimensions. In particular, note that Claim C.1(a) holds iff the distances of all stations to the points $p_{L}$ and $p_{R}$ (the intersection of the $d$-dimensional ball with the $x$-axis) are the same (i.e., $\operatorname{dist}\left(s_{i}, p_{L}\right)=\operatorname{dist}\left(s_{i}, p_{R}\right)$ for every $\left.s_{i} \in S\right)$. In the twodimensional case, this happens only when all stations are aligned on a line (i.e., are $y$-collinear). Generally, in higher dimension $d>2$, this happens when all stations are aligned on a common $(d-1)$-dimensional hyperplane, namely, one dimension lower than the considered space. By the induction assumption for $d-1$, the strong minimum principle holds for any $(d-1)$-dimensional station-free ball, so in particular it holds for the $(d-1)$-dimensional ball $B_{d-1}$ obtained by intersecting the given $d$-dimensional ball $B_{d}$ with the $(d-1)$-dimensional hyperplane in which the stations are embedded. This in turn implies that the center $q$ of the ball $B_{d}$ attains an SINR value strictly better than the minimum value of the points of $\operatorname{bd}\left(B_{d-1}\right) \subset B_{d}$, hence the induction step for $d$ follows.

We conclude this section by proving the No-Free-Hole property, which follows mostly by the fact that the SINR property satisfies the strong minimum principle. Note that the No-Free-Holes property should hold even for reception regions that contain their station. Since the strong minimum principle only applies for regions in which the SINR function is properly defined, and the SINR function is undefined at the positions of the stations, the argument for the NFH property is slightly more delicate.

Corollary 3.16 .

(a) $\left[\right.$ No-Free-Holes in $\mathbb{R}^{d}$. $]$ Let $D \subseteq \mathbb{R}^{d}$ be a compact domain such that $\operatorname{bd}(D) \subseteq \mathcal{H}_{i}(\mathcal{A})$ and $D$ is free from interfering stations (i.e., $D \cap\left(S \backslash\left\{s_{i}\right\}\right)=\emptyset$, albeit $s_{i}$ might be in $\left.D\right)$. Then $D \subseteq \mathcal{H}_{i}(\mathcal{A})$. (b) [Local Minimum points of the SINR function.] The function $\operatorname{SINR}_{\mathcal{A}}\left(s_{i}, p\right)$ has no non-strict local minimum points in $\mathbb{R}^{d} \backslash S$.

Proof. If $s_{i} \notin D$, then Part (a) follows by Theorem 3.1, hence it remains to consider the case where $s_{i} \in D$. Recall that by our definition, $s_{i}$ belongs to $\mathcal{H}_{i}(\mathcal{A})$. Nevertheless, we cannot just rely on this fact and apply Theorem 3.1 to $D \backslash\left\{s_{i}\right\}$, since $D \backslash\left\{s_{i}\right\}$ is not closed, so the theorem does not apply to it. Hence a slightly more delicate argument is needed. Let $B=B\left(s_{i}, \epsilon\right)$ for a sufficiently small $\epsilon>0$ satisfying that $B \subseteq \mathcal{H}_{i}(\mathcal{A}) \cap D$, and consider the region $D^{\prime}=D \backslash B \cup \mathrm{bd}(\mathrm{B})$. It then holds that $D^{\prime}$ is closed and $\operatorname{bd}\left(D^{\prime}\right)=\operatorname{bd}(D) \cup \operatorname{bd}(\mathrm{B}) \subseteq \mathcal{H}_{i}(\mathcal{A})$ and $D^{\prime} \cap S=\emptyset$. Hence by Theorem 3.1, $D^{\prime} \subseteq \mathcal{H}_{i}(\mathcal{A})$. Overall, $D=D^{\prime} \cup B \subseteq \mathcal{H}_{i}(\mathcal{A})$. Part (a) follows.

Next consider Part (b). Assume, towards contradiction, that $\operatorname{SINR}_{\mathcal{A}}\left(s_{i}, p\right)$ has a non-strict local minimum point $p$. Since the SINR function is not defined at the positions of the stations $S$, it holds that $p \notin S$. Let $\beta_{\text {min }}=\operatorname{SINR}_{\mathcal{A}}\left(s_{i}, p\right)$ and let $\epsilon$ be a sufficiently small positive real satisfying that $p$ is the local minimum point in the local ball $B(p, \epsilon)$ around $p$ and in addition $B(p, \epsilon)$ is free from stations. Since $p \notin S$ is a local minimum, such $\epsilon$ exists. Let $p^{\prime} \in \operatorname{bd}(B(p, \epsilon))$ be the point that attains the minimum SINR value on the boundary $\operatorname{bd}(B(p, \epsilon))$. Then, on the one hand, $\operatorname{SINR}_{\mathcal{A}}\left(s_{0}, p\right) \leq$ $\operatorname{SINR}_{\mathcal{A}}\left(s_{0}, p^{\prime}\right)$, since $p$ is a non-strict local minimum in $B(p, \epsilon)$, but on the other hand, since $B(p, \epsilon)$ satisfies the minimum principle, by Theorem $3.1 \operatorname{SINR}_{\mathcal{A}}\left(s_{0}, p\right)>\operatorname{SINR}_{\mathcal{A}}\left(s_{0}, p^{\prime}\right)$, contradiction. Part (b) follows. 


\section{APPLICATIONS}

In this section, we present algorithmic applications for the minimum principle of the SINR function. These applications are based on the practical implications of the minimum principle, namely, there are no free null cells surrounded by a reception region. In addition, it characterizes the minimum points of the SINR function. Most of our applications are based on the characteristic polynomial of $s_{i}$ 's reception region $\mathcal{H}_{i}(\mathcal{A})$. This polynomial is hereafter referred to as the SINR polynomial. For $d=2$, where every station $s_{j} \in S$ is located at point $\left(a_{j}, b_{j}\right)$ in the plane, this polynomial takes the form

$$
\begin{aligned}
\widetilde{H}_{i, \mathcal{A}}(x, y)= & \beta\left[\sum_{j \neq i} \psi_{j} \cdot \prod_{k \neq j}\left(\left(a_{k}-x\right)^{2}+\left(b_{k}-y\right)^{2}\right)+N \prod_{j}\left(\left(a_{j}-x\right)^{2}+\left(b_{j}-y\right)^{2}\right)\right] \\
& -\psi_{i} \prod_{j \neq i}\left(\left(a_{j}-x\right)^{2}+\left(b_{j}-y\right)^{2}\right) .
\end{aligned}
$$

Throughout this section, it is assumed that the stations are located at rational coordinates, which results in a rational SINR polynomial. Moreover, it is assumed that (both the numerator and the denominator of) the transmission powers and coordinates of each station, as well as the threshold $\beta$, are represented with $O(\log n)$ bits. Consequently, a straightforward analysis of Equation (13) reveals the following.

OBSERVATION 4.1. Each coefficient of $\widetilde{H}_{i, \mathcal{A}, \sigma}(x)$ can be represented by $O(n \log n)$ bits.

In many of our applications, we are given a line segment $\sigma=[a, b]$ for rational points $a, b$, and we consider the univariate SINR polynomial $\widetilde{H}_{i, \mathcal{A}, \sigma}(x)$ obtained by restricting the SINR polynomial $\widetilde{H}_{i, \mathcal{A}}(x, y)$ of Equation (13) to $\sigma$.

\subsection{Basic Tools}

In this subsection we describe a collection of tools that utilize the properties of univariate rational polynomials.

The time complexities of all algorithms described in this section are measured in terms of arithmetic operations, i.e., assuming that each arithmetic operation takes $O(1)$ time steps. This model is similar to the one used in $[1,19]$.

Let $\kappa=\min \left\{\operatorname{dist}\left(s_{i}, s_{j}\right) \mid i>1\right\}$ denote the minimum distance between any two stations in the network. Without loss of generality, assume that the minimal transmission power is set to 1 (this can always be obtained by scaling), and let $\Psi_{\max }=\max _{i} \psi_{i}$ be the maximal transmission power. Let

$$
\widetilde{\delta}=\frac{\kappa}{4 \sqrt{\beta \cdot \Psi_{\max } \cdot n}} \text { and } \widetilde{\Delta}=\sqrt{\frac{\Psi_{\max }}{\beta \cdot N}} .
$$

For $\epsilon \in(0,1)$, define $d_{\epsilon}=\epsilon \cdot \widetilde{\delta} / 2$. The following useful facts are taken from [10]. The first fact (slightly modified from [10]) shows that the SINR values of nearby points $p_{1}, p_{2}$ are very close.

ObSERvation 4.2. Let $p_{1}, p_{2}$ be such that $p_{1} \notin \bigcup_{j=1}^{n} B\left(s_{j}, \widetilde{\delta} / 2\right)$ and $p_{2} \in B\left(p_{1}, d_{\epsilon}\right)$. Then $\operatorname{SINR}\left(s_{i}, p_{2}\right) \geq\left(\frac{1-\epsilon}{1+\epsilon}\right)^{\alpha} \cdot \operatorname{SINR}\left(s_{i}, p_{1}\right)$.

Proof. For every station $s_{i}$, as $p_{1} \notin B\left(s_{i}, \widetilde{\delta} / 2\right)$, it holds that

$$
\begin{aligned}
\mathrm{E}_{\mathcal{A}}\left(s_{i}, p_{2}\right) & =\psi_{i} \cdot \operatorname{dist}\left(s_{i}, p_{2}\right)^{-\alpha} \geq \psi_{i} \cdot\left(\operatorname{dist}\left(s_{i}, p_{1}\right)+d_{\epsilon}\right)^{-\alpha} \\
& \geq \psi_{i} \cdot\left((1+\epsilon) \cdot \operatorname{dist}\left(s_{i}, p_{1}\right)\right)^{-\alpha} \geq \frac{\mathrm{E}_{\mathcal{A}}\left(s_{i}, p_{1}\right)}{(1+\epsilon)^{\alpha}}
\end{aligned}
$$


In the same manner, since $p_{1} \notin B\left(s_{j}, \delta / 2\right)$ for every interfering station $s_{j} \neq s_{i}$, we have that

$$
\begin{aligned}
\mathrm{E}_{\mathcal{A}}\left(s_{j}, p_{2}\right) & =\psi_{j} \cdot \operatorname{dist}\left(s_{j}, p_{2}\right)^{-\alpha} \leq \psi_{j} \cdot\left(\operatorname{dist}\left(s_{j}, p_{1}\right)-d_{\epsilon}\right)^{-\alpha} \\
& \leq \psi_{j} \cdot\left((1-\epsilon) \cdot \operatorname{dist}\left(s_{j}, p_{1}\right)\right)^{-\alpha}=\frac{\mathrm{E}_{\mathcal{A}}\left(s_{j}, p_{1}\right)}{(1-\epsilon)^{\alpha}} .
\end{aligned}
$$

Overall,

$$
\operatorname{SINR}\left(s_{i}, p_{2}\right)=\frac{\mathrm{E}_{\mathcal{A}}\left(s_{i}, p_{2}\right)}{\mathrm{I}\left(S \backslash\left\{s_{1}\right\}, p_{2}\right)+N} \geq\left(\frac{1-\epsilon}{1+\epsilon}\right)^{\alpha} \cdot \frac{\mathrm{E}_{\mathcal{A}}\left(s_{i}, p_{1}\right)}{\mathrm{I}\left(S \backslash\left\{s_{i}\right\}, p_{1}\right)+N} \geq\left(\frac{1-\epsilon}{1+\epsilon}\right)^{\alpha} \cdot \operatorname{SINR}\left(s_{i}, p_{1}\right),
$$

yielding our claim.

The following lemma bounds the radii of the largest inscribed circle and the smallest circumscribed circle around $s_{i}$ in $\mathcal{H}_{i}(\mathcal{A})$.

Lemma 4.3 ([10]; Lemma 8.1). Consider a wireless network $\mathcal{A}=\langle d=2, S, \psi, N, \beta, \alpha=2\rangle$. For every station $s_{i} \in S$ :

(a) the radius of the largest inscribed circle around $s_{i}$ in $\mathcal{H}_{i}(\mathcal{A})$ satisfies $\delta\left(s_{i}, \mathcal{H}_{i}(\mathcal{A})\right) \geq \widetilde{\delta}$.

(b) The radius of the smallest circumscribed circle around $s_{i}$ in $\mathcal{H}_{i}(\mathcal{A})$ satisfies $\Delta\left(s_{i}, \mathcal{H}_{i}(\mathcal{A})\right) \leq \widetilde{\Delta}$.

Operations on the univariate characteristic polynomial of the SINR function. A polynomial is said to be square-free if each of its roots has multiplicity one (that is polynomial with no repeated roots $^{7}$ ). The square-free part of a polynomial is a polynomial that has the exact same roots as the original polynomial, each appearing with multiplicity one. In many of our applications, we are given a line segment $\sigma=[a, b]$ for rational numbers $a, b$ and we consider the univariate SINR polynomial $\widetilde{H}_{i, \mathcal{A}, \sigma}(x)$ obtained by restricting the SINR polynomial $\widetilde{H}_{i, \mathcal{A}}(x, y)$ of Equation (13) to $\sigma$. Note that in general $\widetilde{H}_{i, \mathcal{A}, \sigma}(x)$ is not guaranteed to be square-free, however, in $O\left(n \log ^{2} n\right)$ arithmetic operations, one can compute the square-free part of it by using GCD techniques [19].

Counting roots of univariate polynomial within an interval. The Sturm sequence of polynomial $P$ is a sequence $\left\{f_{i}\right\}$ of polynomials such that $f_{0}=P, f_{1}=P^{\prime}$ and such that for every $i \geq 2,-f_{i}$ is the remainder obtained by dividing $f_{i-2}$ by $f_{i-1}$. The Sturm sequence has the property that for every interval $[a, b]$, the number of roots of $P$ in this interval is $Z(a)-Z(b)$, where $Z(x)$ is the number of sign changes in the sequence $\left[f_{0}(x), \ldots, f_{k}(x)\right]$. The Strum sequence can be computed fast using the techniques of [19] in $O\left(n \log ^{2} n\right)$. The Sturm functions can be evaluated at $x$ in $O(n)$ additional time.

THEOREM 4.4 (THEOREM 2 OF [19]). The number of distinct real zeros of a polynomial with rational coefficients of degree $n$, lying in any interval $[a, b]$, can be found with $O(n)$ arithmetic operations, after preprocessing that requires $O\left(n \log ^{2} n\right)$ arithmetic operations.

Root isolation. Isolation of the real roots of a polynomial in a given segment $\sigma$ is the process of finding for each of the real roots a subsegment with rational endpoints containing it and being disjoint from the intervals computed for the other roots (except perhaps for the endpoints of the subsegments). For a given segment $\sigma=[a, b]$, we consider the univariate SINR polynomial $\widetilde{H}_{i, \mathcal{A}, \sigma}(x)$. To avoid complications, we assume that the coordinates of the stations in the network are rational points and that the segment's endpoints $a$ and $b$ are rational points as well. In such a case, the coefficients of the univariate SINR polynomial $\widetilde{H}_{i, \mathcal{A}, \sigma}(x)$ are rational. Our algorithms are

\footnotetext{
${ }^{7}$ For instance, the polynomial $P(x)=(x-a)^{2}$ is not square-free since the root $a$ has multiplicity 2 .
} 
based on isolating the roots of $\widetilde{H}_{i, \mathcal{A}, \sigma}(x)$. Since this polynomial has at most $2 n$ roots, this isolation procedure results in a partition of $\sigma$ into $O(n)$ subsegments, each containing at most one root.

The next theorems are useful in our applications.

THEOREM 4.5 ([4]; REMARK 10.59). Let $P(x)$ be a polynomial of degree $m$ and integer coefficients of bit length at most $\mu$. Then one can isolate the (at most $m$ ) roots of $P(x)$ in $\widetilde{O}\left(m^{2} \mu\right)$ arithmetic operations.

Note that the theorem applies to arbitrary polynomials of degree $m$, and not just square-free polynomials, since in time $O\left(m \log ^{2} m\right)$ one can compute the square-free part of a given polynomial by using GCD techniques [19].

Also note that while the theorem speaks about integer polynomials, it can be applied to rational polynomials, at the cost of a constant increase in the size of the coefficients (noting that the coefficient size equals the sum of the sizes of its numerator and denominator).

The degree of $\widetilde{H}_{i, \mathcal{A}}(x, y)$ is $m=O(n)$, and by Observation 4.1, each of its coefficients has at most $\mu=O(n \log n)$ bits. Hence the theorem implies the following.

COROLlary 4.6. The roots of the polynomial $\widetilde{H}_{i, \mathcal{A}, \sigma}(x)$ can be isolated in $\widetilde{O}\left(n^{3} \log n\right)$ arithmetic operations.

Theorem 4.7 (Approximating All Roots to Within $2^{-q}$, [21]). Let $P(x)$ be a rational polynomial of degree $m$. Then, there exists an algorithm for approximating all roots of $P(x)$ to within $O\left(2^{-q}\right)$ in $\mathrm{O}\left(\mathrm{m}^{2} \cdot q\right)$ arithmetic operations.

\subsection{Reception Testing and Segment Testing}

Throughout this section, assume that the network is embedded in two-dimensional space $(d=2)$. For a given wireless network $\mathcal{A}=\langle d=2, S, \psi, N, \beta, \alpha=2\rangle$ and a target station $s_{i}$, we say that a point $p$ is receptive if $\operatorname{SINR}_{\mathcal{A}}\left(s_{i}, p\right) \geq \beta$. The shape $C$ is receptive if every point $p \in C$ is receptive, i.e., $\operatorname{SINR}_{\mathcal{A}}\left(s_{i}, p\right) \geq \beta$ at every point $p \in C$, otherwise it is non-receptive. In the setting of reception testing, one is given a wireless network $\mathcal{A}$ and a compact domain $C$ that is free from interfering stations, i.e., $C \cap\left(S \backslash\left\{s_{i}\right\}\right)=\emptyset$, and the task is to decide if $C$ is receptive with respect to the station $s_{i}$. The key observation here is that thanks to the minimum principle, it is sufficient to test reception on the boundary of the domain in order to derive conclusions about all its interior points.

Basic Tool: Segment Testing. An important ingredient in our applications is Procedure SegTest, which tests reception on a line segment. To avoid having to deal with errors arising from the inaccuracy of root finding or root isolation algorithms (see Section 4.1 for formal definitions), we assume that the coordinates of the network stations are rational points and in addition that the two points $a$ and $b$ defining the line segment $\sigma=[a, b]$ are rational numbers.

Procedure SegTest receives as input a line segment $\sigma=[a, b]$, a network $\mathcal{A}$ and a target station $s_{i}$, and outputs "yes" if and only if $\sigma \subseteq \mathcal{H}_{i}(\mathcal{A})$. This is done by applying a root isolation procedure on $\widetilde{H}_{i, \mathcal{A}, \sigma}(x)$, i.e., the restriction of the SINR polynomial of Equation (13) to the segment $\sigma$. The output of the root isolation procedure is a partition of $\sigma$ into $O(n)$ disjoint subsegments, each containing at most one root. To decide whether $\sigma$ is receptive or not, Procedure SegTest evaluates the SINR function at the endpoints of each subsegment. The answer is positive if and only if all the endpoints have SINR value at least $\beta$. We next prove the correctness and runtime of Proc. SegTest.

Lemma 4.8. Proc. SegTest outputs "yes" if and only if $\sigma$ is receptive for $s_{i}$, and it operates in $O\left(n^{3}\right.$. $\log n)$ time.

Proof. Let $\sigma^{\prime}=\left[a^{\prime}, b^{\prime}\right]$ be a subsegment containing at most one root $r \neq a^{\prime}, b^{\prime}$ of the restriction of the square-free part of $\widetilde{H}_{i, \mathcal{A}}(x, y)$ on segment $\sigma^{\prime}$. In addition, assume that $\operatorname{SINR}_{\mathcal{A}}\left(s_{i}, c\right)>\beta$ for 
both $c=a^{\prime}$ and $c=b^{\prime}$. As $\sigma^{\prime}$ contains at most one distinct internal root of the square-free part of $\widetilde{H}_{i, \mathcal{A}}(x, y)$ (where the SINR function equals $\beta$ ), it follows that $\sigma^{\prime}$ is receptive.

The time complexity of Proc. SegTest is dominated by the complexity of the root isolation algorithm, see Theorem 4.5.

\subsection{Exact Reception Testing for Polygonal Regions}

Let $\mathcal{P}$ be a polygonal region ${ }^{8}$ free from interfering stations with $m$ vertices located at rational positions in $\mathbb{R}^{2}$. We now describe an exact algorithm for this problem using the minimum principle. Note that the straightforward sampling method does not enable us to decide if the entire polygon is receptive, even when using an arbitrarily large finite set of sampled points in $\mathcal{P}$.

Procedure PolygonRecepTest invokes Proc. SegTest for every edge segment $\sigma \in P$ of $P$ 's boundary and tests its receptiveness for $s_{i}$. It returns "yes" if and only if every edge segment of the polygon is receptive for $s_{i}$. The correctness of Proc. PolygonRecepTest follows immediately by the No-Free-Hole property of Corollary 3.16. We have the following.

Theorem 4.9. Given a polygon $\mathcal{P}$ of $m$ vertices, an $n$-station network $\mathcal{A}$ and target station $s_{i} \in S$, it can be verified in time $O\left(m \cdot n^{3} \log n\right)$ if $\mathcal{P}$ is receptive or not with respect to $s_{i}$.

\section{$4.4 \epsilon$-Approximate Reception Testing for any Shape $(\beta>1)$}

We now consider the general case where the given shape $C$ is a Jordan region in the plane, i.e., an arbitrary compact shape free of interfering stations whose boundary is a Jordan curve. The region $C$ is specified by a parametric description of its boundary curve (Jordan curve), namely, a continuous bijection mapping $f:[0,1] \rightarrow \mathrm{bd}(C)$ such that $f(0)=f(1)$ and moreover, the points $f(z)$ for $z \in_{R}[0,1]$ are uniformly distributed on $\operatorname{bd}(C)$. (One concrete way to do so is by letting $f(z)$ be the point on $\operatorname{bd}(C)$ whose clockwise distance from $f(0)$ on the curve $\operatorname{bd}(C)$ is $z \cdot \operatorname{bd}(C)$ ). In addition, $f(z)$ should be efficiently computable for every $z \in[0,1]$.

In this case, we cannot apply Theorem 4.7, and hence we describe an approximate testing scheme. For some accuracy parameter $\epsilon \in(0,1]$ and target SINR threshold $\beta$, define a compact domain $C$ as $\epsilon$-close to being receptive if for every $p \in C$,

$$
\operatorname{SINR}_{\mathcal{A}}\left(s_{i}, p\right) \geq \beta_{\epsilon},
$$

where

$$
\beta_{\epsilon}=((1-\epsilon) /(1+\epsilon))^{\alpha+1} \cdot \beta
$$

In the setting of approximate reception testing, given a network $\mathcal{A}$, a target station $s_{i} \in S$, a compact domain $C$ free from interfering stations and an accuracy parameter $\epsilon \in(0,1]$, one should decide if the domain $C$ is $\epsilon$-close to being receptive with respect to the station $s_{i}$. In particular, it is required that if the testing procedure returns "yes," then $C$ is $\epsilon$-close to being receptive, and if it says "no," then $C$ is non-receptive. (Note that the answer may leave some uncertainty. In particular, when the algorithm returns "yes", $C$ may still be receptive, and when it returns "no", it is still possible that $C$ is $\epsilon$-close to being receptive.)

Our approximate reception testing scheme tests reception for $s_{i}$ on a sample of points taken from the boundary $\mathrm{bd}(C)$. By the minimum principle property, this would be sufficient to guarantee the reception in the interior of $C$ up to some approximation.

Let $|\mathrm{bd}(C)|$ be the perimeter of $C$, i.e., the length of the boundary curve bd $(C)$. We show the following.

\footnotetext{
$\overline{{ }^{8} \text { As mentioned }}$ before, the method applies to any closed region whose boundary is composed of arcs representable in parametric form by a univariate bounded-degree polynomial.
} 
Theorem 4.10. For a network $\mathcal{A}=\langle d=2, S, \psi, N, \beta>1, \alpha=2\rangle$, an accuracy parameter $\epsilon>0$ and a target station $s_{i}$, there exists an approximate testing procedure ApproxRecepTest that given a compact shape $C \subseteq \mathbb{R}^{2}$ free of stations $s_{j} \neq s_{i}$, returns in $O(n \cdot|\operatorname{bd}(C)| /(\widetilde{\delta} \cdot \epsilon))$ time a binary answer $b_{R}$ such that if $b_{R}=1$ then $C$ is $\epsilon$-close, and if $b_{R}=0$ then $C$ is non-receptive.

In comparison, the straightforward sampling approach (not exploiting the minimum principle) involves sampling points with resolution $\Omega\left(1 / d_{\epsilon}\right)$ and evaluating the SINR function for each point in this sample. Letting area $(C)$ be the area of the domain $C$, the number of sampled points is $\left.\Omega\left(\operatorname{area}(C) / \mathrm{d}_{\epsilon}\right)\right)$. Since each evaluation of the SINR function at a given point takes $O(n)$ time, overall this testing procedure costs $\Omega\left(n \cdot \operatorname{area}(C) / \mathrm{d}_{\epsilon}\right)$. In summary, the time complexity of the testing is a function also of the area of the domain. However, thanks to the minimum principle, it is possible to (approximately) test $C$ in time sublinear in the size of the tested domain, area $(C)$. Specifically, it is sufficient to sample points only from $\operatorname{bd}(C)$, the boundary of $C$. Hence, the testing complexity depends on the perimeter of $C$ instead of its area area $(C)$.

We now describe the approximate testing procedure.

Procedure ApproxRecepTest $\left(\mathcal{A}, s_{i}, C, \epsilon\right)$.

(1) Sample a set of $n_{\epsilon}$ points $P$ evenly spaced on $\operatorname{bd}(C)$, where $n_{\epsilon}=\left\lceil|\mathrm{bd}(C)| / d_{\epsilon}\right\rceil$.

(2) Return 1 if $\operatorname{SINR}_{\mathcal{A}}\left(s_{i}, p\right) \geq \beta$ for every $p \in P$. Otherwise, return 0 .

We now prove the correctness of our scheme.

LEMMA 4.11. receptive.

(a) If ApproxRecepTest $\left(\mathcal{A}, s_{i}, \mathcal{C}, \epsilon\right)$ outputs 1 , then $C$ is $\epsilon$-close to being

(b) If ApproxRecepTest $\left(\mathcal{A}, s_{i}, C, \epsilon\right)$ outputs 0 , then $C$ is non-receptive.

Proof. Suppose ApproxRecepTest $\left(\mathcal{A}, s_{i}, C, \epsilon\right)=1$. Let $P \subseteq \operatorname{bd}(C)$ be the collection of points tested by Procedure ApproxRecepTest. Since ApproxRecepTest $\left(\mathcal{A}, s_{i}, C, \epsilon\right)$ outputs 1 , it follows that for every $p \in P, \operatorname{SINR}_{\mathcal{A}}\left(s_{i}, p\right) \geq \beta$ and since $\beta>1$, by the definition of $\widetilde{\delta}, p \notin \bigcup_{j \neq i} B\left(s_{j}, \widetilde{\delta}\right)$. In addition, for every unsampled boundary point $q \in \operatorname{bd}(C) \backslash P$, it holds that there exists a sufficiently close sampled point $p \in B\left(q, d_{\epsilon}\right) \cap P$. If $p$ is in $B\left(s_{i}, \widetilde{\delta} / 2\right)$, then $q \in B\left(s_{i}, \widetilde{\delta}\right)$, and by Lemma $4.3, \operatorname{SINR}\left(s_{i}, q\right) \geq \beta$ (since the ball of radius $\widetilde{\delta}$ around $s_{i}$ is receptive). Else, we have that $p \notin \bigcup_{s_{j} \in S} B\left(s_{j}, \widetilde{\delta} / 2\right)$ and Observation 4.2 can be safely applied, yielding

$$
\operatorname{SINR}_{\mathcal{H}}\left(s_{i}, q\right) \geq\left(\frac{1-\epsilon}{1+\epsilon}\right)^{\alpha} \cdot \operatorname{SINR}_{\mathcal{H}}\left(s_{i}, p\right) \geq \beta_{\epsilon},
$$

where the last inequality follows by the fact that $\operatorname{SINR}_{\mathcal{A}}\left(s_{i}, p\right) \geq \beta$ and by Equation (15). Since $C$ is free of interfering stations, by Corollary 3.16, it then holds that $\operatorname{SINR}_{\mathcal{A}}\left(s_{i}, q\right) \geq \beta_{\epsilon}$ for every point $q \in C$. By Equation (15), $C$ is $\epsilon$-close to being receptive, establishing Part (a).

Part (b) follows immediately, since the algorithm outputs 0 (in step 2) if there exists a point $p$ on the boundary for which $\operatorname{SINR}_{\mathcal{A}}\left(s_{i}, q\right)<\beta$ and hence $C$ is non-receptive.

Theorem 4.10 follows from Lemma 4.11. We note that our time bounds can be considerably improved into $\widetilde{O}_{\epsilon}(n+|\mathrm{bd}(C)| / \widetilde{\delta})$ by using the efficient approximate batched point location scheme of [2] (Theorem 3.6 therein) in step (1) of Procedure ApproxRecepTest. This, however, requires the reception threshold $\beta$ to be slightly larger, and specifically, we require that $\beta>(1+\epsilon) /(1-\epsilon)$. See Appendix D for the complete scheme.

\subsection{The Maximum Inscribed Reception Sphere}

In this section we consider the task of computing the radius of the maximum inscribed reception sphere $\delta^{*}=\delta\left(s_{i}, \mathcal{H}_{i}(\mathcal{A})\right)$. That is, we would like to compute the maximum radius $\delta^{*}$ satisfying 
that the $d$-dimensional ball of radius $\delta^{*}$ centered at $s_{i}$ is receptive (fully contained in $\mathcal{H}_{i}(\mathcal{A})$ ). For simplicity we consider the two-dimensional case, but the algorithm can be easily adapted to any dimension $d \geq 2$. Consider the wireless network $\mathcal{A}=\langle d=2, S, \psi, N, \beta \geq 1, \alpha=2\rangle$. For an accuracy parameter $\epsilon \in(0,1)$, let $\delta_{\epsilon}^{*}=\delta\left(s_{i}, \mathcal{H}_{i}\left(\mathcal{A}_{\beta_{\epsilon}}\right)\right)$ (see Equation (16)) be the radius of the maximum inscribed reception circle in $\mathcal{H}_{i}\left(\mathcal{A}_{\beta_{\epsilon}}\right)$.

As discussed in Section 4.1, a universal lower bound of $\widetilde{\delta}=\kappa / \sqrt{\beta \cdot \Psi_{\max } \cdot n}$ on $\delta\left(s_{i}, \mathcal{H}_{i}(\mathcal{A})\right)$ is established in [11], by considering the extreme scenario where the station $s_{i}$ is placed at $(0,0)$ with $\psi_{i}=1$ and all other $n-1$ stations are located at $(\kappa, 0)$ with $\psi_{j}=\Psi_{\max }$ for $j \neq i$. For a detailed explanation see Section 8.1 in [11]. In addition, $\widetilde{\Delta}=\sqrt{\Psi_{\max } /(\beta \cdot N)}$ is an upper bound on the maximum inscribed radius. Hence $\widetilde{\delta} \leq \delta^{*} \leq \widetilde{\Delta}$. Algorithm ApproxIncribedCircle searches for an approximation $R_{\mathrm{ALG}}$ for the radius of the maximum inscribed circle in the range $[\widetilde{\delta}, \widetilde{\Delta}]$. It consists of $t=\lceil\log (2 \widetilde{\Delta} / \widetilde{\delta})\rceil$ calls to the reception testing procedure ApproxIncribedCircle of Theorem 4.10. For every $j \in\{0, \ldots, t\}$, let $R_{j}=2^{j} \cdot \widetilde{\delta}$ and let $C_{j}=B\left(s_{i}, R_{j}\right)$ be the ball of radius $R_{j}$ centered at $s_{i}$. Define $j^{\prime} \in\{0, \ldots, t\}$ to be the maximum index satisfying that $C_{j^{\prime}}=B\left(s_{i}, R_{j^{\prime}}\right)$ is non-receptive, i.e., such that ApproxRecepTest $\left(\mathcal{A}, s_{i}, C_{j^{\prime}}, \epsilon\right)=0$. The output of the algorithm is $R_{\mathrm{ALG}}=2^{j^{\prime}-1} \cdot \widetilde{\delta}$.

THEOREM 4.12. Algorithm ApproxIncribedCircle computes in time $O\left(n \cdot \log (\widetilde{\Delta} / \widetilde{\delta}) \cdot \widetilde{\Delta} / d_{\epsilon}\right)$ an approximate radius $R_{\mathrm{ALG}}$ such that $\delta^{*} / 2 \leq R_{\mathrm{ALG}} \leq \delta_{\epsilon}^{*}$, i.e., $B\left(s_{i}, R_{\mathrm{ALG}}\right) \subseteq \mathcal{H}_{i}\left(\mathcal{A}_{\beta_{\epsilon}}\right)$ and $B\left(s_{i}, 2 R_{\mathrm{ALG}}\right) \nsubseteq$ $\mathcal{H}_{i}(\mathcal{A})$.

Proof. We begin by claiming that there exists $j \in\{0, \ldots, t\}$ such that $B\left(s_{i}, R_{j}\right)$ is non-receptive. This holds since $R_{t}=2^{t} \cdot \widetilde{\delta} \geq 2 \widetilde{\Delta}$ and by Lemma 4.3(b), $\widetilde{\Delta}$ is an upper bound on the radius of a receptive circle around $s_{i}$, i.e., $B\left(s_{i}, 2 \widetilde{\Delta}\right)$ is non-receptive. Let $j^{\prime}$ be the last index whose corresponding circle $C_{j^{\prime}}=B\left(s_{i}, R_{j^{\prime}}\right)$ is non-receptive (by the above claim, such a $j^{\prime}$ exists). Hence $R_{\mathrm{ALG}}=R_{j^{\prime}-1}=R_{j^{\prime}} / 2$. Since the ball of radius $\delta^{*}$ is receptive, we conclude that $2 R_{\mathrm{ALG}}=R_{j^{\prime}} \geq \delta^{*}$, establishing the lower bound on $R_{\mathrm{ALG}}$. Turning to the upper bound, note that for $j=0, R_{0}=\widetilde{\delta}$, and by Lemma $4.3(\mathrm{a}), B\left(s_{i}, R_{0}\right)$ is receptive. By the definition of $j^{\prime}$, we have that $B\left(s_{i}, R_{\mathrm{ALG}}\right)$ is $\epsilon$-close to being receptive. Hence $B\left(s_{i}, R_{\mathrm{ALG}}\right) \subseteq \mathcal{H}_{i}\left(\mathcal{A}_{\beta_{\epsilon}}\right)$. Since $B\left(s_{i}, \delta_{\epsilon}^{*}\right)$ is the maximum inscribed ball in $\mathcal{H}_{i}\left(\mathcal{A}_{\beta_{\epsilon}}\right)$, the upper bound follows.

Finally, consider the running time. By Theorem 4.10, Proc. ApproxRecepTest costs $O(n \cdot|\operatorname{bd}(C)| /(\widetilde{\delta} \cdot \epsilon))$ time, where $|\mathrm{bd}(C)|$ is the perimeter of the tested shape. Since Proc. ApproxRecepTest is invoked for circles of radius at most $\widetilde{\Delta}$, each such invocation requires $O\left(n \cdot \widetilde{\Delta} / d_{\epsilon}\right)$ time. As there are $O(\log (\widetilde{\Delta} / \widetilde{\delta}))$ such invocations of Proc. ApproxRecepTest, the claim follows.

In contrast, the basic sampling approach (not relying on the minimum principle) might require us to sample sufficiently many points (with resolution $d_{\epsilon}$ ) in $B\left(s_{i}, \widetilde{\Delta}\right)$ and evaluate the SINR function in each such point, resulting in time complexity $O\left(n \widetilde{\Delta}^{2} /(\widetilde{\delta} \cdot \epsilon)^{2}\right)$.

\subsection{The Polygonal Power Control Problem}

In the feasibility variant of the power control problem, one is given $n$ communication links $\left\langle s_{i}, r_{i}\right\rangle$ and a target SINR threshold $\beta$ and the goal is to compute a feasible power assignment $\psi$ with respect to $\beta$, that is, one that achieves $\operatorname{SINR}\left(s_{i}, r_{i}\right) \geq \beta$ for every $i \in\{1, \ldots, n\}$ when all stations transmit according to it. In the optimization variant of the problem, the parameter $\beta$ is not given; rather, given $n$ communication links $\left\langle s_{i}, r_{i}\right\rangle$, the goal is to compute the maximum SINR threshold $\beta^{*}$ for which there exists a feasible power assignment $\psi^{*}$. Note that the optimization problem can be approximated up to any desired ratio by using an algorithm for the feasibility problem as a 
procedure within an algorithm that computes successively tighter approximations of $\beta$ by binary search.

Note that in the standard setting considered so far, every transmitting station $s_{i}$ was required to be successfully received only at a single reception point $r_{i}$. However, due to stability considerations, communication applications usually require successful reception in a two-dimensional region rather than in a point (or a number of points). In this section, we focus on the feasibility variant of the power control problem and consider a two-dimensional generalization of this problem.

In the Power Control for Polygons (PCPG) problem, one is given a network of $n$ stations $S=\left\{s_{1}, \ldots, s_{n}\right\}$ in the plane, a target SINR threshold $\beta$ and a collection of $n$ polygons $\mathcal{P}_{1}, \ldots, \mathcal{P}_{n}$ that are free from interfering stations (i.e., $P_{i} \cap\left(S \backslash\left\{s_{i}\right\}\right)=\emptyset$ for every $\left.i\right)$. The goal is to find a power assignment $\psi$ satisfying that $\operatorname{SINR}\left(s_{i}, p\right) \geq \beta$ for every $p \in \mathcal{P}_{i}$ and for every $s_{i} \in S$. This yields the following formulation.

Given $\beta, S, N$ and polygons $\mathcal{P}_{1}, \ldots, \mathcal{P}_{n}$, find transmission powers $\psi_{1}, \ldots, \psi_{n}>0$ such that: (17) $\operatorname{SINR}\left(s_{i}, p\right)=\frac{\psi_{i} \cdot \operatorname{dist}\left(s_{i}, p\right)^{-\alpha}}{\sum_{s_{j} \in\left(S \backslash s_{i} \mid\right)} \psi_{j} \cdot \operatorname{dist}\left(s_{j}, p\right)^{-\alpha}+N} \geq \beta$ for every $s_{i} \in S$ and $p \in \mathcal{P}_{i}$.

We next show that Program (17) is convex and in addition it can be solved via the Ellipsoid method despite the fact that it contains infinitely many constraints. This holds since we are able to provide a polynomial separation oracle based on Procedure SegTest discussed above.

Note that with the basic sampling technique, one should sample sufficiently many points inside each polygon $P_{i}$ and solve a linear program consisting of the corresponding SINR constraints. Not only does this approach require a large preprocessing time that depends on the area of the polygons, the number of stations $n$ and the fatness of the reception regions (whose bounds are large), but moreover, it can never guarantee the successful transmission in the entire polygon region, as there is no guarantee that the unsampled polygon points receive the transmission with the desired SINR threshold of $\beta$, but rather with some $\beta-\epsilon$, where $\epsilon \in(0, \beta)$ depends on the density of the sampled points within the polygon. In contrast, using the No-Free-Hole property enables us to provide an exact solution for the PCPG problem for the case where $\beta<\beta^{*}$ or when the optimum power assignment for the given $\beta^{*}$ is rational.

Program (17) consists of infinitely many constraints, namely, an SINR constraint per point $p \in$ $\mathcal{P}_{i}, i \in\{1, \ldots, n\}$. Since $\beta$ is fixed, each such constraint is linear in the $n$ variables $\psi_{1}, \ldots, \psi_{n}$. Hence, the set of feasible power assignments is given by the intersection of (infinitely many) half-planes, we have:

Observation 4.13. Program (17) is convex.

A combinatorial algorithm for exactly solving a convex program is possible only if the problem admits rational solutions [22]. A nonlinear convex program is rational if, for any setting of its parameters to rational numbers such that it has a finite optimal solution, it admits an optimal solution that is rational and can be written using a number of bits that is polynomial in the number of bits needed to write all the parameters. Note that when taking $\beta<\beta^{*}$, there is a continuous non-empty region of feasible powers and in particular there is a rational feasible solution. Hence, if the optimal power assignment for $\beta^{*}$ is rational, our algorithm can compute it exactly.

We now show that this convex program can be solved exactly via the Ellipsoid method despite the fact that it contains infinitely many constraints. This holds since we are able to provide a separation oracle, namely, a polynomial time algorithm that given a candidate solution $\psi^{\prime}$, either determines that $\psi^{\prime}$ is feasible (i.e., satisfies all linear constraints), or returns a violated constraint if $\psi^{\prime}$ is not feasible. The separation oracle in our context is based on Proc. SegTest of Section 4.2. Proc. SegTest is given as input a network $\mathcal{A}$, a target station $s_{i}$ and a line segment $\sigma$, and it decides 
if $\sigma$ is receptive or not. This is done by computing the intersection points of the segment $\sigma$ with the boundary of the reception zone $\operatorname{bd}\left(\mathcal{H}_{i}(\mathcal{A})\right)$. It is easy to see that SegTest can be modified to return a non-receptive point on $\sigma$ if such exists. This non-receptive point is then used to identify a violated constraint to be supplied to the Ellipsoid algorithm.

We now formally describe the separation oracle. Let $\psi^{\prime}$ be a candidate solution (e.g., the center of the current ellipsoid in which the feasible set of solutions reside). Define $\mathcal{A}^{\prime}=\left\langle d, S, \psi^{\prime}, N, \beta, \alpha\right\rangle$. Apply $\operatorname{SegTest}\left(\mathcal{A}^{\prime}, s_{i}, \sigma_{j}\right)$ for every edge $\sigma_{j}$ of the input polygon $P_{i}$ and every $i \in\{1, \ldots, n\}$. If every such $\sigma_{j} \in P_{i}$ is receptive for $s_{i}$, then $\psi^{\prime}$ is a feasible power assignment. Else, let $\sigma_{j} \in P_{\ell}$ be a non-receptive segment with respect to $s_{\ell}$ and let $p \in \sigma_{j}$ be a non-receptive point (this point can be returned by Proc. SegTest). Then, the constraint violated by $\psi^{\prime}$ is $\operatorname{SINR}_{\mathcal{H}^{\prime}}\left(s_{\ell}, p\right) \geq \beta$.

Note that the same scheme can be extended to the sum-power minimization problem or the minmax power problem, in which it is also required to minimize the total (resp., max) transmission power [6]. For example, the Polygonal sum-power minimization problem is formalized as follows.

$$
\begin{aligned}
& \text { Given } \beta, S, N \text {, and polygons } \mathcal{P}_{1}, \ldots, \mathcal{P}_{n}: \text { minimize } \sum_{i=1}^{n} \psi_{i} \text { subject to } \\
& \operatorname{SINR}\left(s_{i}, p\right)=\frac{\psi_{i} \cdot \operatorname{dist}\left(s_{i}, p\right)^{-\alpha}}{\sum_{s_{j} \in\left(S \backslash\left\{s_{i}\right\}\right)} \psi_{j} \cdot \operatorname{dist}\left(s_{j}, p\right)^{-\alpha}+N} \geq \beta \text { for every } s_{i} \in S \text { and } p \in \mathcal{P}_{i} . \\
& \psi_{i} \geq 1, \text { for every } i \in\{1, \ldots, n\} .
\end{aligned}
$$

By the exact same reasoning for Observation 4.13, this program is convex. In addition, the same separation oracle can be used for this problem as well. Thus, as long as the optimal power-assignment is rational, our scheme can compute it in polynomial time, or alternatively, it can converge to this solution by applying binary search on the optimum sum of transmission energies.

We have established the following.

Theorem 4.14. Given a set of $n$ stations $S$, a target SINR threshold $\beta$ and a set of $n$ polygons $\mathcal{P}_{1}, \ldots, \mathcal{P}_{n}$ free of interfering stations (i.e., $\mathcal{P}_{i} \cap\left(S \backslash\left\{s_{i}\right\}\right)=\emptyset$ for every $\left.i\right)$ and whose endpoint vertices are located at rational coordinates, there exists a polynomial time exact algorithm for PCPG when

(a) $\beta<\beta^{*}$ (where $\beta^{*}$ is the optimum SINR threshold of the network), or

(b) $\beta=\beta^{*}$ and the optimum power assignment is rational.

\subsection{Universal Upper Bound for the Number of Null Cells}

We now show that the minimum principle can be utilized for providing a tight linear upper bound on the number of null cells, improving over the $O\left(n^{2 d}\right)$ bound of [11]. Note that the No-Free-Hole property implies that any null cell that is surrounded by a reception cell of station $s_{i}$ contains some interfering station $s_{j}$. See Figure 1 .

This implies that there are at most $O(n)$ null cells in the reception zone of $s_{i}$ and since there are $n$ stations, we have the following immediate corollary.

Corollary 4.15. For every $\beta>0$ and dimension $d \geq 1$, there are $O\left(n^{2}\right)$ null cells for every $n$ station network $\mathcal{A}=\langle d, S, \psi, N, \beta, \alpha=2\rangle$.

In this section, we show that for any $\beta>1$ and dimension $d$ there are in fact only $O(n)$ null cells, and this bound is tight.

Theorem 4.16. For $\beta>1$ and $N>0$, the null zone $\mathcal{H}_{\emptyset}(\mathcal{A}) \subseteq \mathbb{R}^{d}$ contains at most $\tau_{\emptyset}(\mathcal{A})=O(n)$ null cells.

For an illustration of a network with $n-1$ null cells see Figure 1. 
We begin by showing that as long as $N>0$, there exists exactly one infinite null cell and all other cells are bounded.

Lemma 4.17. There exists exactly one unbounded null cell $\mathcal{H}_{\emptyset, j}(\mathcal{A})$.

Proof. Since $N>0$, no station can be received in $\bar{B}=\mathbb{R}^{d} \backslash B\left(s_{0}, r\right)$ for a sufficiently large radius $r$. Therefore, $\bar{B}$ is fully contained in the null zone $\mathcal{H}_{\emptyset}(\mathcal{A})$. (Note also that $\bar{B}$ is clearly connected.)

It remains to bound the number of bounded null cells. We begin by considering the case of $d=2$ and towards the end of this section extend it to general $d$.

For every bounded null cell $H_{j}=\mathcal{H}_{\emptyset, j}(\mathcal{A})$, denote by $J_{j}$ its outer Jordan curve (since a null cell is bounded, this is well-defined). Let $J_{j}^{-}$(resp., $J_{j}^{+}$) denote the region outside (resp., inside) $J_{j}$.

The following two observations play a key role in our analysis. The first concerns reception on the boundary of null cells. We say that a null cell $H_{j}$ enjoys mono-surround if there exists exactly one station $s_{j^{\prime}} \in S$ that is received at every point of the Jordan curve $J_{j}$ of $H_{j}$, namely, such that $J_{j} \subset \mathcal{H}_{j^{\prime}}(\mathcal{A})$. When the null cell $H_{j}$ is mono-surround, we denote by $s\left(J_{j}\right)$ the unique station $s_{j^{\prime}}$ received on the Jordan curve $J_{j}$.

OBSERVATION 4.18. If $\beta>1$, then every bounded null cell $H_{j}$ enjoys mono-surround.

Proof. By definition of the Jordan curve $J_{j}$, some transmission is received at every point $p \in J_{j}$, i.e., for every $p \in J_{j}$ there must exist a station $s_{p}$ such that $\operatorname{SINR}_{\mathcal{A}}\left(s_{p}, p\right) \geq \beta$. By the continuity of $J_{j}$ and since $\beta>1$, this station must be unique, that is, $s_{p_{1}}=s_{p_{2}}$ for every $p_{1}, p_{2} \in J_{j}$, since otherwise there must be a point $p_{0}$ on $J_{j}$ where the identity of the received transmitter switches from some $s_{p_{1}}$ to some other $s_{p_{2}}$, which cannot happen for $\beta>1$.

Note that (perhaps counter-intuitively) it may be possible that $s\left(J_{j}\right) \notin J_{j}^{+}$. For an illustration of this, as well as of Observation 4.18 in general, see Figure 3.

The second observation points out some useful structural relationships between outer Jordan curves of null cells. Two Jordan curves $J_{1}$ and $J_{2}$ are independent if $J_{1}^{+} \nsubseteq J_{2}^{+}$and $J_{2}^{+} \nsubseteq J_{1}^{+}$, otherwise they are dependent. Let $\mathcal{J}=\left\{J_{1}, \ldots, J_{\ell}\right\}$ be the collection of Jordan curves corresponding to the null cells $\mathcal{H}_{\emptyset}(\mathcal{A})=\left\{\mathcal{H}_{\emptyset, 1}(\mathcal{A}), \ldots, \mathcal{H}_{\emptyset, \ell}(\mathcal{A})\right\}$.

ObSERvation 4.19. For every $1 \leq j, j_{1}, j_{2} \leq \ell$,

(a) If $J_{j_{1}}^{+} \cap J_{j_{2}}^{+} \neq \emptyset$ then either $J_{j_{1}} \subset J_{j_{2}}$ or $J_{j_{2}} \subset J_{j_{1}}$.

(b) $J_{j} \cap S=\emptyset$.

(c) $J_{j}^{+}$contains an interfering station $s \neq s\left(J_{j}\right)$, i.e., $\left(S \backslash\left\{s\left(J_{j}\right)\right\}\right) \cap J_{j}^{+} \neq \emptyset$.

Proof. Part (a) holds since two Jordan curves correspond to disjoint null cells and hence they cannot intersect, although they might touch. Hence, the only possible overlap relations between them are that either they are independent or one is contained in the other. Part (b) follows immediately by the fact that $J_{j}$ is the boundary of a null cell. Finally, Part (c) follows by Corollary 3.16.

The null cells digraph. Our analysis is based on inducing a directed forest $F=(\mathcal{J}, A)$ on the set of Jordan curves, where a directed edge $a_{i_{1}, i_{2}} \in A$ connects $J_{i_{1}}$ to $J_{i_{2}}$ iff $J_{i_{2}}^{+} \subset J_{i_{1}}^{+}$and there is no other curve $J_{k}$ satisfying that $J_{i_{2}} \subset J_{k} \subset J_{i_{1}}$. It is easy to verify that $F$ is a directed forest, and each of its components is a tree $T_{i}$ rooted at some $J\left(T_{i}\right)$, with edges directed downwards from the root, where $J_{j}^{+} \subseteq J^{+}\left(T_{i}\right)$ for every curve $J_{j} \in T_{i}$. For an illustration, see Figure 4 .

We may refer to $J \in T_{i}$ as either a vertex in the forest or a curve in the diagram. Let $R_{i}=J^{+}\left(T_{i}\right)$ be the region inside the Jordan curve of the root of $T_{i}$ and let $S_{i}$ be the set of stations restricted to the region $R_{i}$. (Note that $S_{i}$ does not necessarily equal $\left\{s(J) \mid J \in V\left(T_{i}\right)\right\}$.) We bound the number 


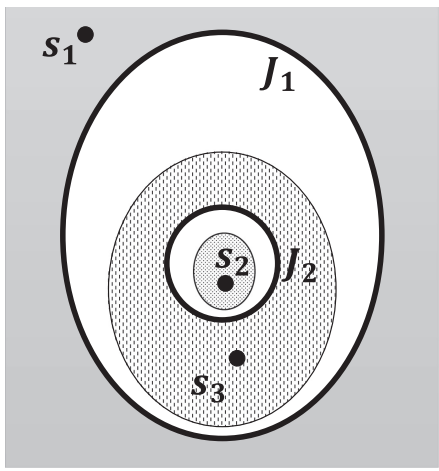

Fig. 3. Illustration of a 3-station network with two null cells, $H_{\emptyset, 1}$ and $H_{\emptyset, 2}$. The strongest station is $s_{1}$ and the weakest is $s_{2}$. Station $s_{1}$ is continuously received on the Jordan curve $J_{1}$. In addition, $s_{3}$ is continuously received on $J_{2}$, despite the fact that $s_{3} \notin J_{2}^{+}$. The Jordan curves $J_{1}$ and $J_{2}$ are dependent, and their null cells digraph corresponds to a single directed edge $\left(J_{1}, J_{2}\right)$.
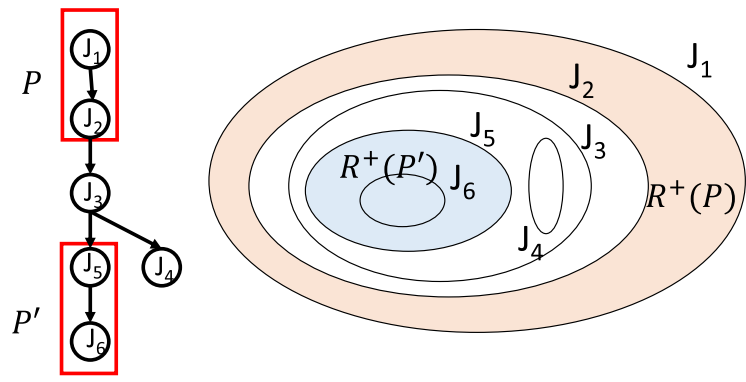

Fig. 4. Schematic illustration of null cells tree $T_{i}$ and the regions associated with the paths of the forest $F_{i}$.

of vertices (i.e., null cells) by showing that for every $i,\left|T_{i}\right| \leq c \cdot\left|S_{i}\right|$ for some constant $c \geq 1$, where $\left|T_{i}\right|$ is the number of vertices in $T_{i}$. Since the sets $S_{i}$ are disjoint (as they reside in disjoint regions $R_{i}$ ), this establishes the desired bound.

From now on, we focus on a specific tree $T_{i}$ and denote by $V_{i}^{\text {high }}$ the vertices with outdegree at least 2 in $T_{i}$ and by $V_{i}^{\text {low }}$ as the complementary set of vertices with outdegree at most 1 . Within this set, let $V_{i}^{\text {leaf }}$ be the set of leaves (outdegree 0 ) in $T_{i}$ and let

$$
V_{\text {leaf }}=\bigcup_{T_{i} \in F} V_{i}^{\text {leaf }}, V_{\text {high }}=\bigcup_{T_{i} \in F} V_{i}^{\text {high }}, V_{\text {low }}=\bigcup_{T_{i} \in F} V_{i}^{\text {low }} .
$$

The edges of the forest $F$ are defined so that the relation " $J_{j} \subset J_{i}$ " is precisely the transitive closure of $F$. It is therefore straightforward to verify that $F$ satisfies the following properties.

ObSERVATION 4.20. (a) If $J_{j} \subset J_{i}$ then $J_{i}$ is an ancestor of $J_{j}$ in $F$.

(b) Every two leaves $J_{k}, J_{k^{\prime}} \in V_{i}^{\text {leaf }}$ are independent.

By Observation 4.20 and the No-Free-Hole property of Corollary 3.16, we bound the total number of high degree vertices in the trees $T_{i}$ by $n$.

LEMMA 4.21. $\left|V_{\text {high }}\right| \leq n$. 
Proof. We first claim that the total number of leaves $\left|V_{\text {leaf }}\right|$ in the forest $F$ is at most $n$. By Corollary 3.16, every $J_{i}$ must contain an interfering station $s_{j} \neq s\left(J_{i}\right)$ inside $J_{i}^{+}$. By Observation 4.20(b), every two leaves $J_{i_{1}}$ and $J_{i_{2}}$ are independent, hence $J_{i_{1}}^{+} \cap J_{i_{2}}^{+}=\emptyset$, implying the claim. The lemma now follows by noting that $\left|V_{i}^{\text {high }}\right| \leq\left|V_{i}^{\text {leaf }}\right|$ for every $T_{i} \in F$.

We now complete the proof of Theorem 4.16 by bounding the total number of low degree vertices in the trees $T_{i}$.

Bounding the set $V_{\text {low. }}$. Let $F_{i}$ be the forest induced by $T_{i} \backslash\left(V_{i}^{\text {high }} \cup\left\{J\left(T_{i}\right)\right\}\right)$. Then $F_{i}$ is a collection of up to $\left|V_{i}^{\text {high }}\right|+2$ vertex-disjoint paths. For every path $P=\left[J_{i_{1}}, \ldots, J_{i_{k}}\right]$ in $F_{i}$, we define two sets of stations, $S_{\text {unique }}(P)$ and $S_{\text {inter }}(P)$, and a multi-set $S_{\text {same }}(P)$. Let

$$
S_{\text {unique }}(P)=\left\{s\left(J_{j}\right) \mid J_{j} \in P \text { and } s\left(J_{j}\right) \neq s\left(J_{k}\right) \text { for every } J_{k} \in P \backslash\left\{J_{j}\right\}\right\} \backslash\left\{s\left(J_{i_{1}}\right)\right\}
$$

be the set of stations that are received on exactly one Jordan curve vertex on $P$ (excluding perhaps the station of the first vertex). Let

$$
S_{\text {same }}(P)=\left\{s(J) \mid J \in P \backslash\left\{J_{1}\right\}\right\} \backslash S_{\text {unique }}(P)
$$

be a multi-set of stations that are received on at least two Jordan curves on $P$. Since $s\left(J_{i^{\prime}}\right)$ is either in $S_{\text {unique }}$ or in $S_{\text {same }}$, for every $J_{i^{\prime}} \in P \backslash\left\{J_{i_{1}}\right\}$, we have that

$$
|P|=\left|S_{\text {unique }}(P)\right|+\left|S_{\text {same }}(P)\right|+1 \text {. }
$$

Finally, we associate two regions with $P$, namely, $R(P)=J_{i_{1}}^{+}$and $R^{+}(P)=R(P) \backslash J_{i_{k}}^{+}$, and let

$$
S_{\text {inter }}(P)=S \cap R^{+}(P)
$$

be the set of stations restricted to the region $R^{+}(P)$. For a schematic illustration see Figure 4 .

LEMMA 4.22. For every $P, P^{\prime} \in F_{i}$, the following properties hold.

(a) $R^{+}(P) \cap R^{+}\left(P^{\prime}\right)=\emptyset$,

(b) $S_{\text {inter }}(P) \cap S_{\text {inter }}\left(P^{\prime}\right)=\emptyset$.

Proof. Let $P=\left[J_{x_{1}}, \ldots, J_{x_{z}}\right]$ and $P^{\prime}=\left[J_{y_{1}}, \ldots, J_{y_{z^{\prime}}}\right]$. By choice, $J_{x_{1}} \neq J_{y_{1}}$. If $J_{x_{1}}$ and $J_{y_{1}}$ are independent, then Part (a) clearly follows. Otherwise, without loss of generality, assume that $J_{y_{1}}^{+} \subset$ $J_{x_{1}}^{+}$(See Figure 4). In such a case, every vertex $J_{x^{\prime}}$ on $P$ satisfies $J_{y_{1}}^{+} \subset J_{x^{\prime}}^{+}$, by Observation 4.20 (a) and since every vertex $J_{x^{\prime}}$ on $P$ has outdegree one. Hence, by Observation $4.20(\mathrm{a}), J_{y_{1}}^{+}$is descendent of $J_{x_{z}}$. Part (a) now follows by noting that $R^{+}(P) \cap J_{x_{z}}^{+}=\emptyset$ but $R^{+}\left(P^{\prime}\right) \subseteq J_{y_{1}}^{+} \subset J_{x_{z}}^{+}$. Part (b) follows directly from Part (a).

LEMMA 4.23. For every directed path from $J_{i_{1}}$ to $J_{i_{2}}$ in $T_{i}$ (i.e., $\left.J_{i_{2}}^{+} \subset J_{i_{1}}^{+}\right)$such that $s\left(J_{i_{1}}\right) \neq s\left(J_{i_{2}}\right)$ :

(a) $s\left(J_{i_{2}}\right) \in J_{i_{1}}^{+}$.

(b) $\psi_{1}>\psi_{2}$, where $\psi_{z}$ is the transmission power of $s\left(J_{i_{z}}\right)$ for $z \in\{1,2\}$.

Proof. For ease of notation, let $s_{i_{1}}=s\left(J_{i_{1}}\right)$ and $s_{i_{2}}=s\left(J_{i_{2}}\right)$. Towards contradiction, assume that $s_{i_{2}} \notin J_{i_{1}}$, and consider the network $\mathcal{A}^{\prime}$ induced on $S^{\prime \prime}=\left(S \backslash S^{\prime}\right) \cup\left\{s_{i_{1}}\right\}$, where $S^{\prime}$ is the set of stations contained in $J_{i_{1}}^{+}$. As $J_{i_{1}}^{+}$is free from interfering stations in $\mathcal{A}^{\prime}$, it follows by Corollary 3.16 that $J_{i_{1}}^{+} \subseteq \mathcal{H}_{i_{1}}\left(\mathcal{A}^{\prime}\right)$. This implies that

$$
\mathrm{E}_{\mathcal{A}}\left(s_{i_{2}}, p\right)=\mathrm{E}_{\mathcal{A}^{\prime}}\left(s_{i_{2}}, p\right)<\mathrm{E}_{\mathcal{A}^{\prime}}\left(s_{i_{1}}, p\right)=\mathrm{E}_{\mathcal{A}}\left(s_{i_{1}}, p\right)
$$

for every point $p \in J_{i_{2}}$, where the strict inequality follows by the fact that $\beta>1$. This contradicts the fact that $s_{i_{2}}$ is received at $p$ in $\mathcal{A}$ and $s_{i_{2}} \neq s_{i_{1}}$. Part (a) follows. For part (b), consider the two station network $\mathcal{A}^{\prime}$ with the stations $S^{\prime}=\left\{s_{i_{1}}, s_{i_{2}}\right\}$. Let $\operatorname{VoR}_{i_{2}}$ be the Voronoi cell of $s_{i_{2}}$ in the 
network of $S^{\prime}$. In such a case, $\operatorname{VoR}_{i_{2}}$ is a half-plane. Let $p \in \operatorname{VoR}_{i_{2}} \cap J_{i_{1}}$. Since $s_{i_{2}} \in J_{i_{1}}^{+}$by part (a), such a point $p$ exists. As $p \in J_{i_{1}}$, it receives $s_{i_{1}}$, hence $\mathrm{E}_{\mathcal{A}}\left(s_{i_{1}}, p\right)>\mathrm{E}_{\mathcal{A}}\left(s_{i_{2}}, p\right)$. On the other hand, $p \in \operatorname{VoR}_{i_{2}}$, and hence $\operatorname{dist}\left(s_{i_{1}}, p\right) \geq \operatorname{dist}\left(s_{i_{2}}, p\right)$. It follows that $\psi_{1}>\psi_{2}$. Part (b) follows.

We next establish an important corollary of Lemma 4.23.

COROLlary 4.24. Let $J_{1}, J_{2}, J_{3} \in \mathcal{J}$ be such that $J_{3}^{+} \subset J_{2}^{+} \subset J_{1}^{+}$, where $s\left(J_{2}\right) \neq s\left(J_{1}\right)$ and $s\left(J_{2}\right) \neq$ $s\left(J_{3}\right)$. Then $s\left(J_{1}\right) \neq s\left(J_{3}\right)$.

Proof. By Lemma 4.23(b) we have that $\psi_{1}>\psi_{2}>\psi_{3}$, so necessarily $s\left(J_{1}\right) \neq s\left(J_{3}\right)$.

LeMma 4.25. $\left|S_{\text {same }}(P)\right| \leq 2\left|S_{\text {inter }}(P)\right|$.

Proof. Let $S_{\text {same }}^{\prime}$ be the unique set (without repetitions) of the multi-set $S_{\text {same }}(P)$. By Corollary 4.24, for every station $s \in S_{\text {same }}^{\prime}$ there is a unique subpath $P_{s} \subseteq P$ such that $s(J)=s$ for every vertex $J \in P_{s}$. These subpaths are disjoint. Define the region of a directed edge $a_{x, y}$ as $R_{x, y}=J_{x}^{+} \backslash J_{y}^{+}$. By the definition of the directed edge, it follows that the regions of any two directed edges $a_{x, y}$ and $a_{x^{\prime}, y^{\prime}}$ for $x \neq x^{\prime}$ are disjoint, i.e., $R_{x, y} \cap R_{x^{\prime}, y^{\prime}}=\emptyset$.

We now show that there exists an interfering station $s^{\prime} \neq s$ in the region $R_{x, y}$ for every $s \in S_{\text {same }}^{\prime}$ and for every directed edge $a_{x, y} \in P_{s}$. To see this, note that as $s\left(J_{x}\right)=s\left(J_{y}\right)=s$, by Corollary 3.16 there must be an interfering station $s_{x, y} \neq s$ in the closed region $R_{x, y}$. Let $S^{\prime}=\left\{s_{x, y} \mid a_{x, y} \in\right.$ $\left.P_{s}, s \in S_{\text {same }}\right\}$. Then $S^{\prime} \subseteq S_{\text {inter }}(P)$, and in addition, $\left|S_{\text {inter }}(P)\right| \geq\left|S^{\prime}\right|=\sum_{s \in S_{\text {same }}^{\prime}}\left(\left|V\left(P_{s}\right)\right|-1\right) \geq$ $\left|S_{\text {same }}(P)\right| / 2$, where the last inequality follows as by definition, $\left|P_{s}\right| \geq 2$ for every $s \in S_{\text {same }}^{\prime}$. The lemma follows.

Next, consider the second set $S_{\text {unique }}(P)$. Recall that $s\left(J_{1}\right) \notin S_{\text {unique }}(P)$ where $J_{1}$ is the first vertex of $P$.

LemMa 4.26. $\quad$ (a) $S_{\text {unique }}(P)$ is inside $R(P)$.

(b) $S_{\text {unique }}\left(P_{1}\right) \cap S_{\text {unique }}\left(P_{2}\right)=\emptyset$ for every distinct $P_{1}, P_{2}$.

Proof. Part (a) follows by Lemma 4.23 since every vertex $J \in P$ with $s(J) \in S_{\text {unique }}(P)$ satisfies that $s(J) \neq s\left(J_{1}\right)$ and there is a directed path from $J_{1}$ to $J$. Now consider Part (b). Let $S_{1}=S_{\text {unique }}\left(P_{1}\right)$ and $S_{2}=S_{\text {unique }}\left(P_{2}\right)$. Assume, towards contradiction, that there exists some station $s_{j}$ in the intersection $S_{1} \cap S_{2}$. Let $J_{j} \in P_{1}$ and $J_{k} \in P_{2}$ be such that $s_{j}$ is the station received on $J_{j}$ and $J_{k}$, i.e.,

$$
s_{j}=s\left(J_{j}\right)=s\left(J_{k}\right) \text {. }
$$

By part (a), $s_{j} \in R\left(P_{1}\right), R\left(P_{2}\right)$, hence $P_{1}$ and $P_{2}$ are dependent. Without loss of generality, assume that $R\left(P_{1}\right) \subset R\left(P_{2}\right)$. Hence, there is a directed path from $J_{k}$ to $J_{j}$ that goes through $J_{m}$, where $J_{m}$ is the first vertex of $P_{1}$. Since $s\left(J_{j}\right)$ is unique in $P_{1}$, it holds that $s\left(J_{m}\right) \neq s\left(J_{j}\right)$ (as both $J_{j}$ and $J_{m}$ are on $P_{1}$ ) and by Equation (19), also $s\left(J_{m}\right) \neq s\left(J_{k}\right)$. Contradiction follows by Corollary 4.24 (setting $J_{1}=J_{k}, J_{2}=J_{m}, J_{3}=J_{j}$ ).

LEMMA 4.27. $\left|V_{\text {low }}\right|=O(n)$.

Proof. For every $P$, let $S^{+}(P)=S_{\text {inter }}(P) \cup S_{\text {unique }}(P)$. Since the stations of $S^{+}(P)$ reside in $R(P)$, it holds that for every $P \in F_{i}$ and $P^{\prime} \in F_{j}$, where $i \neq j, S^{+}(P)$ and $S^{+}\left(P^{\prime}\right)$ are disjoint. In addition, for two paths $P$ and $P^{\prime}$ that are in the same forest $F_{i}$, Lemma 4.25 implies that $S_{\text {inter }}(P)$ and $S_{\text {inter }}\left(P^{\prime}\right)$ are disjoint, and Lemma 4.26 implies that $S_{\text {unique }}(P)$ and $S_{\text {unique }}\left(P^{\prime}\right)$ are disjoint as well. 
From now on, we consider only paths $P \in F_{i}$ of length at least 2 . Note that there are at most $O\left(\left|V_{\text {leaf }}\right|\right)=O(n)$ paths of length 1 , hence those paths would increase the bound by an additive factor of at most $O(n)$. Let

$$
\mathcal{P}=\left\{P \subseteq F_{i}|| P \mid \geq 2 \text { for every } T_{i} \subseteq F\right\}
$$

be the collection of paths considered. Define

$$
V_{\text {low }}^{-}=\bigcup_{P \in \mathcal{P}} V(P), \mu_{1}=\sum_{P \in \mathcal{P}}\left|S_{\text {unique }}(P)\right|, \mu_{2}=\sum_{P \in \mathcal{P}}\left|S_{\text {same }}(P)\right| .
$$

By Equation (18), and since every $P \in \mathcal{P}$ is of length at least 2, it holds that

$$
\left|V_{\text {low }}^{-}\right| \leq 2\left(\left|\mu_{1}\right|+\left|\mu_{2}\right|\right) \text {. }
$$

By Lemma 4.26(b), $\mu_{1}=\left|\bigcup_{P \in \mathcal{P}} S_{\text {unique }}(P)\right| \leq n$. In addition, by Lemmas 4.25 and $4.22(\mathrm{~b})$,

$$
\mu_{2}=\sum_{P \in \mathcal{P}}\left|S_{\text {same }}(P)\right| \leq 2 \sum_{P \in \mathcal{P}}\left|S_{\text {inter }}(P)\right|=2\left|\bigcup_{P \in \mathcal{P}} S_{\text {inter }}(P)\right| \leq 2 n .
$$

We have that $\left|V_{\text {low }}^{-}\right| \leq 6 n$. Recall that there are at most $O\left(\left|V_{\text {leaf }}\right|\right)=O(n)$ paths of length 1 that are omitted from $\mathcal{P}$ and hence from $V_{\text {low }}^{-}$, thus $\left|V_{\text {low }}\right| \leq\left|V_{\text {low }}^{-}\right|+O(n) \leq 6 n+O(n)$.

The lemma follows.

We are now ready to complete the proof of Theorem 4.16.

Proof of Theorem 4.16. For $d=2$ the theorem follows by Lemmas 4.21 and 4.27. To bound the number of null cells for the general case of $d \geq 2$, the following definitions are now extended. Any bounded null cell $\mathcal{H}_{\emptyset, j}$ is defined by a set of points for which all $n$ SINR functions hold with strict inequalities (i.e., $\operatorname{SINR}\left(s_{i}, p\right)<\beta$ ). Hence, the path from any point $p \in \mathcal{H}_{\emptyset, j}$ to a sufficiently far away point $q$ intersects the surface where one of the SINR inequality first becomes an equality Since $\beta>1$, by continuity for all points $p \in \mathcal{H}_{\emptyset, j}$ the curve satisfies the same SINR equality and this is the boundary $J_{j}$ of the null cell. In other words, $J_{j}$ is the boundary (where SINR equality is achieved) of the topological closure of the cell (i.e., the union of the open set where the inequality is satisfied along with the boundary where equality is satisfied). The notion of boundary replaces the terminology of a Jordan curve used in $d=2$ ). Let $s\left(J_{j}\right)$ be the station received on $J_{j}$ (i.e., letting $s_{j^{\prime}}=s\left(J_{j}\right)$ then $\left.J_{j} \subset \mathcal{H}_{j^{\prime}}\left(\mathcal{A}^{\prime}\right)\right)$. By Observation 4.18, this is well defined. Note that any path from $p \in \mathcal{H}_{\emptyset, j}$ to an infinite (i.e., sufficiently far-away) point $q$ intersects the boundary $J_{j}$ (i.e., where the SINR inequalities of station $s\left(J_{j}\right)$ becomes an equality). Let $J_{j}^{+}$be the open set of $J_{j}$ (since $J_{j}$ is defined by equality of one of the SINR functions, its interior is an open set). In other words, $J_{j}^{+}$contains all points $p$ for which every path to a point $q$ at infinity intersects the boundary $J_{j}$. Analogously to the two-dimensional case, we say that two cells $H_{1}=\mathcal{H}_{\emptyset, j_{1}}(\mathcal{A})$ and $H_{2}=\mathcal{H}_{\emptyset, j_{2}}(\mathcal{A})$, with outer boundaries $J_{1}$ and $J_{2}$, respectively, are dependent if $J_{1}^{+} \subset J_{2}^{+}$or vice-verse. To apply the proof of the two-dimensional case, it is then sufficient to establish Observation 4.19(a) for general $d$. (Claims (2) and (3) are extended naturally to $d \geq 2$.)

Proof of Observation 4.19(A) For Every Dimension $d \geq 2$. We would like to show that if the topological closures of two cells $\mathcal{H}_{\emptyset, 1}, \mathcal{H}_{\emptyset, 2}$ intersect in interior point $p \in J_{1}^{+} \cap J_{2}^{+}$, then either $J_{1} \subseteq J_{2}$ or vice versa.

Since $p$ is in $J_{k}^{+}$, every path from $p$ to a point $q$ at infinity (i.e., sufficiently far away point) must intersect the boundary $J_{k}$ for $k \in\{1,2\}$. In contrast for a point $p^{\prime} \notin J_{k}^{+}$, there exists a path from $p^{\prime}$ to $q$ that does not intersect $J_{k}$ for $k \in\{1,2\}$. Therefore, every path from the mutual point $p$ to a 
point $q$ at infinity intersects both of the boundaries $J_{1}$ and $J_{2}$. Without loss of generality, assume that it intersects $J_{1}$ first, we claim that $J_{1}^{+} \subseteq J_{2}^{+}$.

Suppose towards contradiction that there is $p^{\prime} \in J_{1}^{+} \backslash J_{2}^{+}$. Hence, there exists a path from $p^{\prime}$ to a point at infinity $q$ that does not intersect $J_{2}$ and as a result also a path from $p$ to $p^{\prime}$ (as both of these points are in $J_{1}^{+}$) that does not intersect $J_{2}$. Contradiction to the fact the $p \in J_{2}^{+}$.

After establishing Observation 4.19, we can safely define a null cells digraph for the collection of $d$-dimensional null cells. The proof arguments on that graph are invariant to dimension. Theorem 4.16 follows.

\subsection{Approximation of the Number of Null Cells for Any $\beta>0$}

This universal linear bound is tight in the sense that there are networks with $\Omega(n)$ null cells. However, for networks with $O(1)$ null cells, the bound of $O(n)$ is a poor approximation. We now propose a scheme that approximates the number of null cells with the guarantee that every null cell in the map is detected.

To this end, we use the following notation. Let $\mathcal{A}_{\beta^{\prime}}$ be a network identical to $\mathcal{A}$ except its SINR threshold is $\beta^{\prime}$ instead of $\beta$. To avoid cumbersome notation, we focus on the station $s_{i}$ and define $\tau\left(\beta^{\prime}, i\right)$ as the number of null cells (holes) surrounded by the reception cells of $s_{i}$ in the network $\mathcal{A}_{\beta^{\prime}}$. For additional notation necessary for this section, see Section 4.1. In this section, we present a scheme that given an approximation parameter $\epsilon \in(0,1)$ and a target station $s_{i}$, returns an approximation $X(i, \epsilon)$ for $\tau(\beta, i)$, satisfying that $\tau(\beta, i) \leq X(i, \epsilon) \leq \tau\left(\beta_{\epsilon}, i\right)$, for $\beta_{\epsilon}$ defined in Equation (16). For $\beta>1$, it then holds that

$$
\tau_{\emptyset}\left(\mathcal{A}_{\beta}\right) \leq \sum_{i=1}^{n} X(i, \epsilon) \leq \tau_{\emptyset}\left(\mathcal{A}_{\beta_{\epsilon}}\right),
$$

where $\tau_{\emptyset}(\mathcal{A})$ is the total number of null cells in the network $\mathcal{A}$.

We remark that the scheme described in this section applies to the setting where $\beta>1$. This is because the crucial property of mono-surround, established in the previous section (Lemma 4.18), stating that exactly one station is received on the boundary of every null cell, is guaranteed only when $\beta>1$. In contrast, when $0<\beta \leq 1$, it might be the case that different regions on the boundary of the null cell are received by different stations and hence the minimum principle (e.g., the No-Free-Hole property) cannot be directly applied. We note however that the presented scheme can also be applied for a setting where $\beta \leq 1$, by slightly modifying the definition of a null cell and focusing on a given fixed station. Specifically, for a given station $s_{i}$ and any $\beta>0$, our scheme can count the number of "holes" in $s_{i}$ 's map, defined as the connected regions in which $s_{i}$ cannot be correctly received, i.e., the scheme approximates the number of connected regions in $\mathbb{R}^{d} \backslash \mathcal{H}_{i}\left(\mathcal{A}_{\beta}\right)$.

We begin by showing, using the minimum principle, that the number of null cells (i.e., holes) surrounded by the reception region $\mathcal{H}_{i}\left(\mathcal{A}_{\beta}\right)$ is monotonically decreasing with $\beta$. Note that this property does not hold for the number of reception cells in $\mathcal{H}_{i}\left(\mathcal{A}_{\beta}\right)$. In particular, in the extreme cases where $\beta$ is either infinitely small or extremely large, $\mathcal{H}_{i}\left(\mathcal{A}_{\beta}\right)$ consists of one connected reception cell.

Lemma 4.28. Let $0<\beta_{1} \leq \beta_{2}$. Then $\tau\left(\beta_{1}, i\right) \geq \tau\left(\beta_{2}, i\right)$.

Proof. By the minimum principle, every null cell in the map of $s_{i}$ contains an interfering station $s_{j} \in S \backslash\left\{s_{i}\right\}$, hence there are at most $n-1$ bounded null cells plus one infinite null cell in $\mathcal{H}_{i}\left(\mathcal{A}_{\beta}\right)$ for every $\beta>0$. (This is tight for a sufficiently small $\beta$.) Let $H_{1}, \ldots, H_{\ell}$ be the null cells in $\mathcal{H}_{i}\left(\mathcal{A}_{\beta_{2}}\right)$ such that $H_{j}$ contains the interfering station $s_{k_{j}} \neq s_{i}$. By the definition of null cells, the interfering stations $s_{k_{j}}$ are distinct. We now show that for every $1 \leq j \leq \ell$, there exists a corresponding null cell $H_{j}^{\prime}$ in $\mathcal{H}_{i}\left(\mathcal{A}_{\beta_{1}}\right)$ that contains $s_{k_{j}}$. By definition, the boundary of the null cell $H_{j}$ satisfies 
$\operatorname{bd}\left(H_{j}\right) \subseteq \mathcal{H}_{i}\left(\mathcal{A}_{\beta_{2}}\right)$ and $s_{k_{j}} \in H_{j}$. Since $\beta_{1} \leq \beta_{2}$, we get that $\operatorname{bd}\left(H_{j}\right) \subseteq \mathcal{H}_{i}\left(\mathcal{A}_{\beta_{1}}\right)$ as well, and since $s_{j} \in H_{j}$ is a point that belongs to a null cell surrounded by $s_{i}$ 's reception region $\mathcal{H}_{i}\left(\mathcal{A}_{\beta}\right)$ for every $\beta>0$, there exists a null cell $H_{j}^{\prime}$ in $\mathcal{H}_{i}\left(\mathcal{A}_{\beta_{1}}\right)$ that contains $s_{k_{j}}$. The lemma follows.

Note that without the minimum principle, one encounters two main difficulties when approximating the number of null cells. First, since a priori the null cell can be located anywhere in the map, one has to sample $\Omega(\widetilde{\Delta} /(\epsilon \cdot \widetilde{\delta}))$ points in the disk defined by the minimum bounding circle of $\mathcal{H}_{i}\left(\mathcal{A}_{\beta}\right)$ and evaluate the SINR function on each of those points. In addition, this brute-force scheme cannot detect all null cells in the reception zone $\mathcal{H}_{i}\left(\mathcal{A}_{\beta}\right)$ as there might be infinitesimally small null cells that are not captured by the sampling. However, thanks to the minimum principle, and in particular the No-Free-Hole property, every null cell must contain an interfering station and hence the interfering stations can be a useful starting point for detecting the null cells in the map. In addition, as shown next, the minimum principle also implies that the null cells cannot be too small, hence there exists a fixed sampling precision that guarantees the detection of every null cell in $\mathcal{H}_{i}\left(\mathcal{A}_{\beta}\right)$.

LEMmA 4.29. For every $\beta>0$, the area of every null cell is $\Omega\left(\kappa^{2} \cdot \widehat{\beta} /\left(\Psi_{\max } \cdot n\right)\right)$, where $\widehat{\beta}=$ $\min \{\beta, 1 / \beta\}$.

Proof. By the No-Free-Hole property, every null cell contains some interfering station $s_{j} \in$ $S \backslash\left\{s_{i}\right\}$. Let $\delta^{\prime}=\kappa /\left(4 \sqrt{\Psi_{\max } \cdot n}\right)$. By Lemma 4.3 , the ball $B_{\beta}=B\left(s_{j}, \delta^{\prime} / \sqrt{\beta}\right)$ is fully contained in $\mathcal{H}_{j}\left(\mathcal{A}_{\beta}\right)$. Hence, for $\beta \geq 1$ and every $i \neq j$, it holds that $B_{\beta} \cap \mathcal{H}_{i}\left(\mathcal{A}_{\beta}\right)=\emptyset$, implying that the null cell containing $s_{j}$ contains also all of $B_{\beta}$, and the lemma follows. It remains to consider the case where $\beta<1$. Note that in this case that ball $B_{1 / \beta}$ is fully contained in $\mathcal{H}_{j}\left(\mathcal{A}_{1 / \beta}\right)$, hence in particular, $\mathrm{E}\left(s_{j}, p\right)>(1 / \beta) \cdot \mathrm{E}\left(s_{i}, p\right)$ for every $i \neq j$ and $p \in B_{1 / \beta}$. We conclude that $B_{1 / \beta} \cap \mathcal{H}_{i}\left(\mathcal{A}_{\beta}\right)=\emptyset$. The lemma follows for this case as well.

The Algorithm. We now present Algorithm ApproxHoles that approximates the number of null cells (holes) in the reception region of station $s_{i}$. Moreover, the algorithm enjoys the following properties.

(Q1) Every null cell in $\mathcal{H}_{i}\left(\mathcal{A}_{\beta}\right)$ is detected.

(Q2) Every detected null cell exists in $\mathcal{H}_{i}\left(\mathcal{A}_{\beta_{\epsilon}}\right)$.

Algorithm ApproxHoles uses an implicit grid tessellation of the network. Given a point $p \in \mathbb{R}^{2}$, the grid cell to which $p$ belongs can be computed in $O(1)$ time. The grid resolution $\delta_{G}$ is defined by

$$
\delta_{G}=\sqrt{\min \{1 / \beta, \beta\}} \cdot \widetilde{\delta} /(6 \epsilon),
$$

where $\widetilde{\delta}$ is defined in Equation (14). The algorithm consists of two phases, a tagging phase and a hole-recognition phase. In the tagging phase, the grid cells that intersect the boundaries of the null cells in the map $\mathcal{H}_{i}\left(\mathcal{A}_{\beta}\right)$ ), as well as their 8-neighborhoods, are detected and marked. Then, in the hole-recognition phase, the algorithm traverses the tagged cells and evaluates the number of null cells in them.

We begin by describing the tagging phase. The algorithm classifies the grid cells into three types: white, gray and black. Initially all grid cells are white. A grid cell is colored gray when the algorithm finds out that it intersects $\operatorname{bd}\left(\mathcal{H}_{i}\left(\mathcal{A}_{\beta}\right)\right)$. To decide if a given grid cell $C$ intersects $\operatorname{bd}\left(\mathcal{H}_{i}\left(\mathcal{A}_{\beta}\right)\right)$, the algorithm applies Sturm procedure of Theorem 4.4 on the restriction of the polynomial $\widetilde{H}_{i, \mathcal{A}_{\beta}}(x, y)$ defined in Equation (13) on each of the edges of $C$. Later, in Lemma 4.30, we show that since the algorithm attempts to reconstruct only the boundaries of the null cells in $\mathcal{H}_{i}\left(\mathcal{A}_{\beta}\right)$ (and not the entire boundary $\left.\operatorname{bd}\left(\mathcal{H}_{i}\left(\mathcal{A}_{\beta}\right)\right)\right)$, it is indeed sufficient to test only the edges of $C$. The algorithm 
begins by computing an initial collection of gray cells. This is done by using Theorem 4.7 to evaluate, for every interfering station $s_{j} \in S \backslash\left\{s_{i}\right\}$, the roots of the polynomial $\widetilde{H}_{i, \mathcal{A}_{\beta}, \sigma_{i, j}}(x)$ up to an approximation factor of $O\left(\delta_{G}\right)$, where $\sigma_{i, j}$ is the line segment connecting $s_{i}$ and $s_{j}$. The grid cells that contain the approximated roots of these polynomials are colored gray, as well as the grid cells in their 8-neighborhoods. To explore the boundaries of the null cells, the algorithm repeatedly explores every gray cell $C$, by testing its 8-neighborhood for intersection with $\operatorname{bd}\left(\mathcal{H}_{i}\left(\mathcal{A}_{\beta}\right)\right)$. At the end of $C$ 's exploration, the white cells in its 8-neighborhood that intersect bd $\left(\mathcal{H}_{i}\left(\mathcal{A}_{\beta}\right)\right)$ are colored gray and the explored gray cell $C$ is colored black. (That is, after the exploration, the gray cell $C$ becomes black but this may end with new gray cells in the 8-neighborhood of $C$.) This exploration process continues as long as unexplored gray cells exist. At the end of the tagging phase, there are no gray grid cells, and each black grid cell has a nearby point on $\operatorname{bd}\left(\mathcal{H}_{i}\left(\mathcal{A}_{\beta}\right)\right)$ (this intersection point is either on the grid cell or on one of its eight neighboring grid cells). To increase the security region, the algorithm colors in black the 8-neighborhood of each black grid cell. This completes the description of the tagging phase.

The second phase, namely, the hole-recognition phase, exploits the tagging to recognize null cells in $\mathcal{H}_{i}\left(\mathcal{A}_{\beta}\right)$. The input for this phase is a collection of black grid cells $B L$ computed by the tagging phase. To this collection, we also add the set $S Q$ of $O(\widetilde{\Delta})$ grid cells that intersect with the circumference of the $\widetilde{\Delta} \times \widetilde{\Delta}$ square $Q$ centered at $s_{i}$. (By Lemma 4.3(b), $\mathcal{H}_{i}\left(\mathcal{A}_{\beta}\right)$ is fully contained in $Q$.) The algorithm then defines a planar subdivision $\mathcal{M}$ induced by the collection of $B L \cup S Q$ grid cells (i.e., the vertices of these grid cells along with their edges). It then employs the algorithm of [18] to construct a point-location data-structure DS that maintains $\mathcal{M}$. This DS supports pointlocation queries on $\mathcal{M}$, that is, given a point $p$, it returns the (unique) region in $\mathcal{M}$ that contains $p$. The final output of the algorithm is computed by applying $n-1$ point-location queries to DS for each interfering station and returning the number of distinct regions (i.e., point-location answers) that are occupied by these stations.

Analysis. Let $H_{1}, \ldots, H_{k}$ be the collection of null cells in $s_{i}$ 's map. We first show that it is sufficient to test the edges of the grid cells for intersection with $\operatorname{bd}\left(\mathcal{H}_{i}\left(\mathcal{A}_{\beta}\right)\right)$.

LEMma 4.30. Every grid cell that intersects the boundary of a null cell in $\operatorname{bd}\left(\mathcal{H}_{i}\left(\mathcal{A}_{\beta}\right)\right)$ must intersect it with one of its edges.

Proof. Consider a grid cell $C$ that none its edges intersects bd $\left(\mathcal{H}_{i}\left(\mathcal{A}_{\beta}\right)\right)$. This implies that each of the four edges is either positive, namely, contained in $\mathcal{H}_{i}\left(\mathcal{A}_{\beta}\right)$, or negative, i.e., none of its points is in $\mathcal{H}_{i}\left(\mathcal{A}_{\beta}\right)$. We consider the following cases.

Case 1: $C$ contains an interfering station $s_{j}$. By Lemma 4.3(a), the entire grid cell is fully contained in $\mathcal{H}_{j}\left(\mathcal{A}_{\beta}\right)$, and hence does not intersect the null cell.

Case 2: $C$ is free from interfering stations. By the continuity of the SINR function on the boundary of $C$, it holds that either all four edges of $C$ are positive or all are negative.

Case 2.1 : all four edges of $C$ are negative. There are two sub-cases to consider here. First assume that $C \cap \mathcal{H}_{i}\left(\mathcal{A}_{\beta}\right)=\emptyset$ for every station $s_{i}$. In this case, $C$ is strictly contained in a null cell, i.e., does not intersect with any null cell, and claim holds.

Otherwise, there is an internal reception cell inside $C$, that is, there exists $s_{i}$ such that $C \cap$ $\mathcal{H}_{i}\left(\mathcal{A}_{\beta}\right) \neq \emptyset$. Since by Lemma 4.29 , the area of a null cell is greater than $\delta_{G}$, no null cell is fully contained in $C$, hence the claim follows for this case as well.

Case 2.2: all four edges of $C$ are positive. Since $C$ contains no interfering station, by the minimum principle, the entire grid cell is fully contained in the reception zone $\mathcal{H}_{i}\left(\mathcal{A}_{\beta}\right)$, and in particular, there exists no interior point $p$ inside the grid cell $C$ such that $p \in \operatorname{bd}\left(\mathcal{H}_{i}\left(\mathcal{A}_{\beta}\right)\right)$. The lemma follows. 
We proceed by showing that at the end of the tagging phase, every point on the boundary of every null cell is contained in some black grid cell.

Lemma 4.31. For every $\ell \in\{1, \ldots, k\}$, and every $p \in \mathrm{bd}\left(H_{\ell}\right)$, the grid cell containing $p$ is black.

Proof. We consider some fixed connected component of the boundary of bd $\left(H_{\ell}\right)$, denoted by $\operatorname{bd}_{y}\left(H_{\ell}\right)$. Let $s_{j} \in S \backslash\left\{s_{i}\right\}$ be some interfering station located inside the region enclosed by $\mathrm{bd}_{y}\left(H_{\ell}\right)$. By the No-Free-Hole property, such $s_{j}$ exists. Then the line segment $\sigma_{i, j}$ between $s_{i}$ and $s_{j}$ must intersect $\mathrm{bd}_{y}\left(H_{\ell}\right)$ at some point $q$. Note that this point is also the root of the polynomial $\widetilde{H}_{i, \mathcal{A}, \sigma_{i, j}}(x)$. Consider some point $p$ in $\operatorname{bd}_{y}\left(H_{\ell}\right)$ and let $C_{1}, \ldots, C_{x}$ be the grid cells that intersect with the continuous curve that connects $q$ and $p$ on $\operatorname{bd}_{y}\left(H_{\ell}\right)$ such that $C_{1}$ (resp., $C_{x}$ ) contains $q$ (resp., $p$ ). We prove, by induction on $t \in\{1, \ldots, x\}$, that $C_{1}, \ldots, C_{t}$ are tagged by the algorithm as black.

For the base of the induction, consider the grid cell $C_{1}$ that contains $q$. We show that this cell was tagged as gray (and hence also as black after the algorithm explored it). Since the algorithm computes the roots of $\widetilde{H}_{i, \mathcal{A}, \sigma_{i, j}}(x)$ up to an approximation factor of $\delta_{\epsilon}$, it computes an approximate root $q^{\prime}$ satisfying that $\operatorname{dist}\left(q, q^{\prime}\right) \leq \delta_{\epsilon}$. In particular, $q$ resides either in the grid cell of $q^{\prime}, C_{q^{\prime}}$ or in the 8-neighborhood of $C_{q^{\prime}}$. Since the algorithm tags $C_{q^{\prime}}$ and its 8-neighborhood as gray, $C_{q}$ is tagged as gray. The induction base holds. Now assume the claim holds up to $t>1$ and consider the grid cell $C_{t+1}$. By the induction assumption, $C_{t}$ is tagged as black and hence it was also tagged as gray earlier. Recall that every gray cell is explored, i.e., its 8 neighborhood is tested for intersection with $\mathcal{H}_{i}\left(\mathcal{A}_{\beta}\right)$. Since $C_{t+1}$ is in the 8-neighborhood of $C_{t}$, the algorithm tests if at least one of its four edges intersects $\mathcal{H}_{i}\left(\mathcal{A}_{\beta}\right)$ and if so, it is tagged as gray. By Lemma 4.30, since $C_{t+1}$ intersects $\mathcal{H}_{i}\left(\mathcal{A}_{\beta}\right)$, one of its edges intersects it as well, which will be discovered by algorithm. This proves the inductive step. The lemma follows.

The following claim justifies the selection of the grid resolution.

Claim 4.32. Every grid edge in $\mathcal{M}$ is fully contained in $\mathcal{H}_{i}\left(\mathcal{A}_{\beta_{\epsilon}}\right)$.

Proof. Every edge $e$ taken into $\mathcal{M}$ is an edge of a black cell $C_{1}$. This implies that there exists a point $p \in \operatorname{bd}\left(\mathcal{H}_{i}\left(\mathcal{A}_{\beta}\right)\right)$ that belongs either to $C_{1}$ or to one of its 24 distance- 2 neighbors (comprising the security area taken around each intersected grid cell). We now show that $p \notin \bigcup_{j=1}^{n} B\left(s_{j}, 5 \cdot \delta_{G}\right)$. This holds since every station $s_{j}$ is received successfully in its $r$-ball around it for $r=\sqrt{\min \{1 / \beta, \beta\}}$. $\widetilde{\delta}$. Hence, no point $p^{\prime} \in B\left(s_{j}, 5 \cdot \delta_{G}\right)$ can be on the boundary $\mathcal{H}_{i}\left(\mathcal{A}_{\beta}\right)$ for every $j$. In addition, since $\operatorname{dist}(p, q) \leq 5 \delta_{G}$ for every point $q \in e$, by the proof of Observation 4.2 , the claim follows.

For every null cell $H_{\ell}$ in $\mathcal{H}_{i}\left(\mathcal{A}_{\beta}\right)$, let $C_{\ell}=\left\{C \in B L \mid C \cap \operatorname{bd}\left(H_{\ell}\right) \neq \emptyset\right\}$ be the collection of the black cells that intersect bd $\left(H_{\ell}\right)$. By Lemma 4.31, the black cells of $C_{\ell}$ cover the boundary bd $\left(H_{\ell}\right)$. Let $E_{\ell}$ be the grid edges that are incident to a black cell in $C_{\ell}$ and to a white cell inside $H_{\ell}$. By the lower-bound on the size of a null cell stated in Lemma 4.29 and the selection of the grid resolution $\delta_{G}$, every $H_{\ell}$ contains at least one interior white cell. Hence $E_{\ell}$ contains a cycle of length at least 4 that is contained in $H_{\ell}$.

We now present additional useful properties of $E_{\ell}$.

Lemma 4.33. For every null cell $H_{\ell}$,

(a) $E_{\ell}$ defines a bounded polygon that is fully contained in $H_{\ell}$.

(b) $E_{\ell} \subseteq \mathcal{H}_{i}\left(\mathcal{A}_{\beta_{\epsilon}}\right)$.

(c) Every interfering station inside $H_{\ell}$ is inside $E_{\ell}$ as well.

Proof. Part (a) follows by definition and Cl. 4.31. Part (b) follows by Claim 4.32. Finally, by the proof of $\mathrm{Cl}$. 4.31, every point $p$ that is located between $\operatorname{bd}\left(H_{\ell}\right)$ and $E_{\ell}$ is in $\mathcal{H}_{i}\left(\mathcal{A}_{\beta}\right)$. Hence, $p \notin S \backslash\left\{s_{i}\right\}$. The lemma follows. 
We proceed by showing that Algorithm ApproxHoles satisfies properties (Q1) and (Q2).

LEMMA 4.34. For every pair of interfering stations $s_{j} \neq s_{k} \in S \backslash\left\{s_{i}\right\}$ :

(a) If $s_{j}$ and $s_{k}$ occur in distinct null cells in $\mathcal{H}_{i}\left(\mathcal{A}_{\beta}\right)$, then they belong to distinct regions in $\mathcal{M}$.

(b) If $s_{j}$ and $s_{k}$ occur in distinct regions in $\mathcal{M}$, then they belong to distinct null cells in $\mathcal{H}_{i}\left(\mathcal{A}_{\beta_{\epsilon}}\right)$.

Proof. For $t \in\{j, k\}$, let $H_{i_{t}}$ be the null cell containing $s_{t}$ in $\mathcal{H}_{i}\left(\mathcal{A}_{\beta}\right)$. Since $H_{i_{j}}$ and $H_{i_{k}}$ are distinct null cells, their interior regions do not overlap, and hence their interior bounded polygons $E_{i_{j}}$ and $E_{i_{k}}$ do not overlap. Recall that by Lemma 4.33, $s_{t}$ is contained in $E_{i_{t}}$ for $t \in\{j, k\}$, and since these polygons $E_{i_{j}}$ and $E_{i_{t}}$ are part of the planar subdivision map $\mathcal{M}$, it holds that $s_{j}$ and $s_{k}$ belong to distinct regions in $\mathcal{M}$. Part (a) follows. Consider Part (b). Let $F_{t}$ be the polygon (or face) to which $s_{t}$ belongs in $\mathcal{M}$. By Claim 4.32 , the edges that define $F_{t}$ are fully contained $\mathcal{H}_{i}\left(\mathcal{A}_{\beta_{\epsilon}}\right)$. Since both $F_{j}$ and $F_{k}$ contain an interfering station, each of them contains a null cell in $\mathcal{H}_{i}\left(\mathcal{A}_{\beta_{\epsilon}}\right)$. The claim follows immediately as the regions of $F_{j}$ and $F_{k}$ do not overlap.

Finally, we bound the run-time of the algorithm. First we consider the tagging phase and bound the number of grid cells that intersect the boundary of $s_{i}$ 's reception zone.

ObServation 4.35. At the end of the tagging phase, there are $O\left(\left|\operatorname{bd}\left(\mathcal{H}_{i}\left(\mathcal{A}_{\beta}\right)\right)\right| / \delta_{G}\right)$ black cells.

Proof. Let $X$ be the set of black cells that intersect the boundary $\operatorname{bd}\left(\mathcal{H}_{i}\left(\mathcal{A}_{\beta}\right)\right)$. Since every cell has 8 neighboring cells, and since every black cell has a neighboring black cell in $X$, it suffices to establish an asymptotic bound on $|X|$. The 9-block of a grid cell $C$, denoted $\Gamma_{9}(C)$, is the collection of nine grid cells surrounding $C$, i.e., $C$ and its 8-neighborhood. Let $B(X)$ be the set of 9-blocks of the cells of $X$. Note that total number of non-overlapping 9-blocks in $B(X)$ is bounded by $O\left(\left|\operatorname{bd}\left(\mathcal{H}_{i}\left(\mathcal{A}_{\beta}\right)\right)\right| / \delta_{G}\right)$, because the distance between any two points $p_{1} \in C_{1} \cap \operatorname{bd}\left(\mathcal{H}_{i}\left(\mathcal{A}_{\beta}\right)\right)$ and $p_{2} \in C_{2} \cap \operatorname{bd}\left(\mathcal{H}_{i}\left(\mathcal{A}_{\beta}\right)\right)$ is at least $\delta_{G}$. Since every 9-block intersects with a constant number of other 9-blocks, we get that $|B(X)|=O\left(\left|\operatorname{bd}\left(\mathcal{H}_{i}\left(\mathcal{A}_{\beta}\right)\right)\right| / \delta_{G}\right)$ and consequently also $|X|=$ $O\left(\left|\operatorname{bd}\left(\mathcal{H}_{i}\left(\mathcal{A}_{\beta}\right)\right)\right| / \delta_{G}\right)$.

Lemma 4.36. Algorithm ApproxHoles runs in time $O\left(n^{3} / \delta_{G}+n \cdot\left|\operatorname{bd}\left(\mathcal{H}_{i}\left(\mathcal{A}_{\beta}\right)\right)\right| / \delta_{G}+n \cdot \widetilde{\Delta}\right)$.

Proof. The tagging phase begins by evaluating the roots of the $n-1$ polynomials $\widetilde{H}_{i, \mathcal{A}_{\beta}, \sigma_{i, j}}(x)$ up to an approximation factor of $O\left(\delta_{G}\right)$, where $\sigma_{i, j}$ is the line segment connecting $s_{i}$ and $s_{j}$ for every $s_{j} \in S \backslash\left\{s_{i}\right\}$. Since the degree of each such polynomial is $O(n)$, by Theorem 4.7, this costs $O\left(n^{3} / \delta_{G}\right)$ time. Let $|B L|$ be the total number of black cells. By Theorem 4.4, following an $O(n \log n)$ time preprocessing stage, each application of Sturm procedure (see Theorem 4.4) on grid cell edges costs $O(n)$ time. Hence overall, the run time of the tagging phase is $O\left(n^{3} / \delta_{G}+|B L| \cdot n\right)$. The construction of the map $\mathcal{M}$ is linear in the cardinality of its vertex set $V(\mathcal{M})=B L \cup S Q$, and by Observation 4.35, $|V(\mathcal{M})|=O\left(\left|\operatorname{bd}\left(\mathcal{H}_{i}(\mathcal{A})\right)\right|+\widetilde{\Delta}\right)$. Each point location query costs $O(\log (|V(\mathcal{M})|))$ time, and since there are $n-1$ such queries, one per interfering station, the total time complexity is $O(n \log (\mid V(\mathcal{M} \mid)))$.

By Lemma 4.34 and Lemma 4.36, we have the following.

THEOREM 4.37. There exists an algorithm that given an approximation parameter $\epsilon \in(0,1)$, a network $\mathcal{A}_{\beta}$ and a target station $s_{i}$, returns a number $X(i, \epsilon)$ such that $\tau(\beta, i) \leq X(i, \epsilon) \leq \tau\left(\beta_{\epsilon}, i\right)$ in $O\left(n^{3} /(\widetilde{\delta} \cdot \epsilon)+n \cdot\left|\operatorname{bd}\left(\mathcal{H}_{i}(\mathcal{A})\right)\right| /(\epsilon \cdot \widetilde{\delta})+\widetilde{\Delta} \cdot n\right)$ time. Moreover, the algorithm satisfies properties $(Q 1)$ and (Q2). 


\section{APPENDICES}

\section{A A TECHNICAL TOOL}

The following technical proposition plays a key role in our analysis.

Proposition A.1. For all $x \in(0,1), y_{1}, \ldots, y_{n} \in(0,1)$, and $a_{1}, \ldots, a_{n}, \alpha \in \mathbb{R}_{>0}$,

$$
\max \left\{\sum_{i=1}^{n} a_{i}\left(\frac{x}{y_{i}}\right)^{\alpha}, \sum_{i=1}^{n} a_{i}\left(\frac{1-x}{1-y_{i}}\right)^{\alpha}\right\} \geq \sum_{i=1}^{n} a_{i} .
$$

where equality holds iff $x=y_{1}=\cdots=y_{n}$.

Proof. We begin by formulating the inequality as a lower bound on the solution to an optimization problem. Fix some $x \in(0,1)$ and let $Y=\left(y_{1}, \ldots, y_{n}\right)$,

$$
F(Y)=\sum_{i=1}^{n} a_{i}\left(\frac{x}{y_{i}}\right)^{\alpha} \text { and } G(Y)=\sum_{i=1}^{n} a_{i}\left(\frac{1-x}{1-y_{i}}\right)^{\alpha} .
$$

It is easy to verify that the function $F(Y)$ (respectively, $G(Y))$ is continuous and strictly decreasing (resp., increasing) in all variables $y_{1}, \ldots, y_{n}$. Therefore, $\max \{F(Y), G(Y)\}$ is minimized when $F(Y)=G(Y)$. Let $H(Y)=F(Y)-G(Y)$, and consider the following optimization problem.

$$
\Pi: \min \{F(Y)\} \text {, such that } H(Y)=0 \text { and } \quad Y \in(0,1)^{n} .
$$

Define the contour of $\Pi$ by

$$
H_{0}=\left\{Y \in(0,1)^{n} \mid H(Y)=0\right\} .
$$

Note that to prove Equation (A.1), it suffices to show that the solution to problem $\Pi$ is at least $\sum_{i=1}^{n} a_{i}$.

To prove the lemma, we study the structure of $H_{0}$, beginning with the following observation.

Observation A.2. For any given fixed values of $y_{1}, \ldots, y_{j-1}, y_{j+1}, \ldots, y_{n} \in(0,1)$, there exists a unique value of $y_{j}$ such that the point $Y=\left(y_{1}, \ldots, y_{j}, \ldots, y_{n}\right) \in H_{0}$.

Proof. Consider some index $j \in\{1, \ldots, n\}$, fix the values of $y_{i} \in(0,1)$ for every $i \neq j$, and consider the constant $c_{0}=\sum_{i \neq j} \frac{a_{i}}{a_{j}}\left[\left(\frac{1-x}{1-y_{i}}\right)^{\alpha}-\left(\frac{x}{y_{i}}\right)^{\alpha}\right]$ and the function $f\left(y_{j}\right)=\left(\frac{x}{y_{j}}\right)^{\alpha}-\left(\frac{1-x}{1-y_{j}}\right)^{\alpha}$. For a given value of $y_{j}$, the point $Y=\left(y_{1}, \ldots, y_{n}\right)$ satisfies $H(Y)=0$ if and only if $f\left(y_{j}\right)=c_{0}$. Since the function $f\left(y_{j}\right)$ is continuous and strictly decreasing in $y_{j} \in(0,1)$ with $\lim _{y_{j} \rightarrow 0+} f\left(y_{j}\right)=+\infty$ and $\lim _{y_{j} \rightarrow 1-} f\left(y_{j}\right)=-\infty$, it follows that there exists a unique $y_{j} \in(0,1)$ such that $f\left(y_{j}\right)=c_{0}$. Observation A.2 follows.

From now on, we focus on the restriction $F_{0}$ of the function $F(Y)$ to the contour $H_{0}$. Let $F_{0}$ : $H_{0} \rightarrow \mathbb{R}$ where $F_{0}(Y)=F(Y)$ for every $Y \in H_{0}$.

Lemma A.3. The function $F_{0}$ has a stationary point $Y_{\min } \in H_{0}$ which is a global minimum point in $H_{0}$.

Proof. Consider the point $Y^{\prime}=(x, \ldots, x)$ for the $x \in(0,1)$ fixed earlier. Since $F\left(Y^{\prime}\right)=G\left(Y^{\prime}\right)$, it holds that $Y^{\prime} \in H_{0}$. For a sufficiently small $\epsilon>0$, let $B_{\epsilon}\left(Y^{\prime}\right)$ be the set of $H_{0}$ points that fall in the ball of radius $r_{\epsilon}=\max \{x, 1-x\}-\epsilon$ around $Y^{\prime}$ in the $L_{\infty}$ norm. Formally,

$$
B_{\epsilon}\left(Y^{\prime}\right)=\left\{Y \in H_{0} \mid\left\|Y-Y^{\prime}\right\|_{\infty} \leq r_{\epsilon}\right\} .
$$

Let $\operatorname{bd}\left(B_{\epsilon}\left(Y^{\prime}\right)\right)=\left\{Y \in H_{0} \quad \mid \quad\left\|Y-Y^{\prime}\right\|_{\infty}=r_{\epsilon}\right\}$ be the boundary of the ball. Note that by Observation A.2, $\operatorname{bd}\left(B_{\epsilon}\left(Y^{\prime}\right)\right) \neq \emptyset$. (I.e., for example, if $x<1 / 2$, then there exists some $y_{1}$ satisfying that the point $Y^{\prime \prime}=\left(y_{1}, x, \ldots, x, 1-\epsilon-x\right)$ is in $\left.\operatorname{bd}\left(B_{\epsilon}\left(Y^{\prime}\right)\right)\right)$.

Claim A.4. There exists an $\epsilon>0$ for which $F_{0}(\widehat{Y}) \geq F\left(Y^{\prime}\right)$ for every $\widehat{Y} \in \operatorname{bd}\left(B_{\epsilon}\left(Y^{\prime}\right)\right)$. 
Proof. First consider the case where $x<1 / 2$, hence $r_{\epsilon}=1-x-\epsilon$. Fix a sufficiently small $0<\epsilon<1 / 2-x$ satisfying that $x<r_{\epsilon}$, and consider a point $\widehat{Y} \in \operatorname{bd}\left(B_{\epsilon}\left(Y^{\prime}\right)\right)$. By definition, there exists at least one coordinate in $\widehat{Y}$, say $\widehat{y}_{j}$, for which $\left\|\widehat{y}_{j}-x\right\|=r_{\epsilon}=1-x-\epsilon$. Since $x<r_{\epsilon}$, necessarily $\widehat{y}_{j}=x+r_{\epsilon}=1-\epsilon$. By tending $\epsilon$ to $0, G(\widehat{Y})$ tends to infinity (as it has a summand $\left.\left((1-x) /\left(1-\widehat{y}_{j}\right)\right)^{\alpha} \cdot a_{i}=((1-x) / \epsilon)^{\alpha} \cdot a_{i}\right)$. Hence, for sufficiently small $\epsilon, F_{0}(\widehat{Y})>F_{0}\left(Y^{\prime}\right)$ and more generally, $\lim _{\epsilon \rightarrow 0} \min \left\{F_{0}(\widehat{Y}) \mid \widehat{Y} \in \operatorname{bd}\left(B_{\epsilon}(Y)\right)\right\}=\infty$.

Next, consider the case where $x>1 / 2$, hence $r_{\epsilon}=x-\epsilon$, and consider a point $\widehat{Y} \in \operatorname{bd}\left(B_{\epsilon}\left(Y^{\prime}\right)\right)$ for a sufficiently small $\epsilon<2 x-1$ satisfying that $x+r_{\epsilon}>1$. Let $\widehat{Y} \in \operatorname{bd}\left(B_{\epsilon}\left(Y^{\prime}\right)\right)$. By definition, there exists at least one coordinate in $\widehat{Y}$, say $\widehat{y}_{j}$, for which $\left\|\widehat{y}_{j}-x\right\|=r_{\epsilon}=x-\epsilon$. Since $x+$ $r_{\epsilon}>1$, it follows that $\widehat{y}_{j}=\epsilon$. Hence, $F_{0}(\widehat{Y})>F_{0}\left(Y^{\prime}\right)$ for a sufficiently small $\epsilon$. More generally, $\lim _{\epsilon \rightarrow 0} \min \left\{F^{\prime}(\widehat{Y}) \mid \widehat{Y} \in \operatorname{bd}\left(B_{\epsilon}\left(Y^{\prime}\right)\right)\right\}=\infty$.

Finally, consider the case where $x=1 / 2$. Any point $\widehat{Y} \in \operatorname{bd}\left(B_{\epsilon}\left(Y^{\prime}\right)\right)$ has a coordinate $\widehat{y}_{j}$ such that $\left\|\widehat{y}_{j}-x\right\|=r_{\epsilon}$ and hence $\widehat{y}_{j} \in\{\epsilon, 1-\epsilon\}$. Hence, $F_{0}(\widehat{Y})>F_{0}\left(Y^{\prime}\right)$ for a sufficiently small $\epsilon>0$. Claim A.4 follows.

Fix $\epsilon$ as in Claim A.4. The function $F_{0}$ is continuous in the closed and bounded interval $B_{\epsilon}\left(Y^{\prime}\right)$. By the extreme value theorem (Chapter 2 of [14], Theorem 2.4.15 of [20]) the function $F_{0}$ attains a global maximum $Y_{\max }$ and a global minimum $Y_{\min }$ at least once in $B_{\epsilon}\left(Y^{\prime}\right)$, i.e., such that $F_{0}\left(Y_{\min }\right) \leq$ $F_{0}(Y) \leq F_{0}\left(Y_{\max }\right)$ for every $Y \in B_{\epsilon}\left(Y^{\prime}\right)$. By Claim A.4, there is a minimum point $Y_{\min }$ of $F_{0}$ which is strictly in the open interval, inside the ball $B_{\epsilon}\left(Y^{\prime}\right)$. Therefore, as the function $F_{0}$ is differentiable at $Y_{\min }$, it holds by Fermat's theorem (Chapter 11 of [20]) that $Y_{\min }$ is a stationary point of $F_{0}$, i.e., all its partial derivatives are zero at that point, establishing Lemma A.3.

We are now ready to complete the proof by showing that $Y^{\prime}=(x, \ldots, x)$ is the global minimum point of $F_{0}$.

LemmA A.5. The point $Y^{\prime}=(x, \ldots, x) \in H_{0}$ is a global minimum of $F_{0}$ in $H_{0}$, i.e., $F_{0}(Y) \geq F_{0}\left(Y^{\prime}\right)$ for every $Y \in H_{0}$.

Proof. By Lemma A.3, the function $F_{0}$ has a stationary point $Y_{\min } \in H_{0}$ which is a global minimum point.

To find a stationary point for $F_{0}(Y)$ it is required to find the minimum of $F(Y)$ for $Y \in(0,1)^{n}$ subject to the constraint that $H(Y)=0$. To do that, we consider the Lagrange function $L(Y, \lambda)=$ $F(Y)-\lambda \cdot H(Y)$. We now claim that the point $\left(Y^{\prime}, \lambda^{\prime}\right)$ where $y_{1}^{\prime}=x, \ldots, y_{n}^{\prime}=x, \lambda^{\prime}=1-x$ is a stationary point of $L(Y, \lambda)$. To see this, consider the derivative of $L$ with respect to the $n+1$ variables $y_{1}, \ldots, y_{n}, \lambda$.

$$
\frac{\partial L(Y, \lambda)}{\partial y_{i}}=\alpha \cdot a_{i} \cdot\left(\lambda \cdot \frac{(1-x)^{\alpha}}{\left(1-y_{i}\right)^{\alpha+1}}+(\lambda-1) \cdot \frac{x^{\alpha}}{y_{i}^{\alpha+1}}\right)
$$

for every $i=1, \ldots, n$ and

$$
\begin{array}{r}
\frac{\partial L}{\partial \lambda}=-H\left(y_{1}, \ldots, y_{n}\right) \\
=\sum_{i=1}^{n} a_{i}\left[\left(\frac{x-1}{y-1}\right)^{\alpha}-\left(\frac{x}{y}\right)^{\alpha}\right] .
\end{array}
$$

Observation A.6. Any stationary point $(\tilde{Y}, \tilde{\lambda})$ for $L(Y, \lambda)$ satisfies $\tilde{\lambda}=1-x$. 
Proof. First, note that by Equation (A.4), $\frac{\partial L}{\partial y_{i}}(Y, \lambda) \neq 0$ for $\widetilde{\lambda} \in\{0,1\}$. Since $(\widetilde{Y}, \widetilde{\lambda})$ is a stationary point, by plugging it into Equation (A.4) and equating it to zero, we have that $\frac{x^{\alpha}}{y_{i}^{\alpha+1}}=\frac{\tilde{\lambda}}{1-\tilde{\lambda}} \cdot \frac{(1-x)^{\alpha}}{\left(1-y_{i}\right)^{\alpha+1}}$, hence

$$
\left(\frac{x}{y_{i}}\right)=\left(\frac{\tilde{\lambda} \cdot x}{(1-\tilde{\lambda})(1-x)}\right)^{1 /(\alpha+1)}\left(\frac{1-x}{1-y_{i}}\right)
$$

for every $i=1, \ldots, n$. Plugging this into Equation (A.5) and equating to zero, we get that

$$
\frac{\partial L}{\partial \lambda}(\tilde{Y}, \tilde{\lambda})=\left(\left(\frac{\tilde{\lambda} \cdot x}{(1-\tilde{\lambda})(1-x)}\right)^{\alpha /(\alpha+1)}-1\right) \cdot \sum_{i=1}^{n} a_{i} \cdot\left(\frac{1-x}{1-y_{i}}\right)^{\alpha}=0 .
$$

Since $a_{i}>0$, for every $i$, it must hold that $\lambda \cdot x /((1-\lambda)(1-x))-1=0$, hence $\tilde{\lambda}=1-x$. Observation A.6 follows.

Finally, using Observation A.6 and plugging $\lambda=1-x$ in the $n$ functions of Equation (A.4), it holds that $y_{i}=x$ for every $i \in\{1, \ldots, n\}$, so $Y=Y^{\prime}$ as required. Lemma A.5 follows.

Since $F_{0}\left(Y^{\prime}\right)=\sum_{i=1}^{n} a_{i}$, by Lemma A.5, it follows that the solution to the problem $\Pi$ of Equation (A.2) is at least $\sum_{i=1}^{n} a_{i}$, hence Equation (A.1) holds and Proposition A.1 is established.

\section{B DISCRETE AND CONTINUOUS AVERAGE STRENGTH}

All angles considered hereafter are integer multiples of $\theta \in(0,2 \pi)$ for some positive integer $k$. Given a vector $\Theta=\left(\theta_{1}, \ldots, \theta_{d-1}\right)$ consisting of $d-1$ angles $\theta_{1}, \ldots, \theta_{d-1} \in(0,2 \pi)$, let $p(\Theta)=$ $p\left(\theta_{1}, \ldots, \theta_{d-1}\right)=\left(x_{1}, \ldots, x_{d-1}\right) \in \operatorname{bd}(\mathrm{B})$ be a point on the boundary of the $d$-dimensional ball B where $x_{1}=r \cos \left(\theta_{1}\right)$ and $x_{j}=r \sin \left(\theta_{1}\right) \cdot \ldots \cdots \sin \left(\theta_{j-1}\right) \cdot \cos \left(\theta_{j}\right)$ for $j \in\{2, \ldots, d-1\}$ and $x_{d}=x_{d-1} \cdot \tan \left(\theta_{d-1}\right)$ (indeed, one can verify that $\left.\sum_{j=1}^{d} x_{j}^{2}=r^{2}\right)$. Let

$$
\Phi=\left\{\Delta \Theta=\left(\Delta \theta_{1}, \ldots, \Delta \theta_{d-1}\right) \mid 2 \pi / \Delta \theta_{i} \in \mathbb{N}_{\geq 1} \text { for every } i\right\}
$$

be the collection of angle vectors whose components are integral fractions of $2 \pi$. (Later on, we are interested in what happens when the angles $\Delta \theta$ tend to zero.)

For the $d$-ball $\mathrm{B}=\mathrm{B}(q, r)$ and for every positive integral vector $\bar{X}=\left(\chi_{1}, \ldots, \chi_{d-1}\right) \in \mathbb{N}_{\geq 1}^{d-1}$, define the discrete boundary of B by

$$
\operatorname{bd}^{\text {discr }}(B, \bar{X})=\left\{p\left(i_{1} \cdot \Delta \theta_{1}, \ldots, i_{d-1} \cdot \Delta \theta_{d-1}\right) \mid i_{1} \in\left\{0, \ldots, \chi_{1}-1\right\}, \ldots, i_{d-1} \in\left\{0, \ldots, \chi_{d-1}-1\right\}\right\},
$$

where $\Delta \theta_{i}=2 \pi / \chi_{i}$ for every $i \in\{1, \ldots, d-1\}$. The discrete boundary bd ${ }^{\text {discr }}(B, \bar{X})$ consists of a sample of $\chi=\prod_{i=1}^{d-1} \chi_{i}$ "receiver" points positioned on $\operatorname{bd}(\mathrm{B})$. The vector $\bar{X}=\left(\chi_{1}, \ldots, \chi_{d-1}\right)$ then defines the resolution of the discrete boundary of $\operatorname{bd}(\mathrm{B})$. Note that by definition, the vector of angles $\Delta \Theta=\left(\Delta \theta_{1}, \ldots, \Delta \theta_{d-1}\right)$ satisfies $\Delta \Theta \in \Phi$. We also define the rational boundary of the ball $\mathrm{B}$ as the union of all discrete boundaries of $\mathrm{B}$, formally given by

$$
\operatorname{bd}^{\text {ratio }}(\mathrm{B})=\bigcup_{\chi_{1}=1}^{\infty} \bigcup_{\chi_{2}=1}^{\infty} \cdots \bigcup_{\chi d-1}^{\infty} \operatorname{bd}^{\text {discr }}(B, \bar{X}) \text {. }
$$

It then holds that the (continuous) boundary of B is the closure of its rational boundary, i.e.,

$$
\operatorname{bd}(\mathrm{B})=\mathrm{cl}\left(\mathrm{bd}^{\text {ratio }}(\mathrm{B})\right) \text {. }
$$

For every station $s_{j} \in S, d$-ball $\mathrm{B}=\mathrm{B}(q, r)$ and $\bar{X}=\left(\chi_{1}, \ldots, \chi_{d-1}\right) \in \mathbb{N}_{\geq 1}^{d-1}$, let $\varepsilon_{j}(q, r, \bar{X})$ denote the discrete average strength of $s_{i}$ experienced at the points of the discrete boundary bd ${ }^{\text {discr }}(B, \bar{X})$ 
by

$$
\varepsilon_{j}(q, r, \bar{X})=\frac{1}{\chi} \cdot \sum_{p \in \operatorname{bd}^{\mathrm{discr}}(B, \bar{X})} \frac{\psi_{i}}{\operatorname{dist}\left(s_{j}, p\right)^{2}} .
$$

For the continuous boundary, the average strength of $s_{j}$ experienced at the boundary bd(B) of $\mathrm{B}=\mathrm{B}(q, r)$, denoted by $\varepsilon_{j}(q, r)$, is given as the limit of $\varepsilon_{j}(q, r, \bar{X})$ when $\chi_{i}$ tends to infinity for every $i$, i.e.,

$$
\varepsilon_{j}(q, r)=\lim _{\bar{X} \in \mathbb{N}_{\geq 1}^{d-1},\|\bar{X}\|_{1} \rightarrow \infty} \varepsilon_{j}(q, r, \bar{X}) .
$$

We now extend Claim 7.4 of [11] for the case of general dimension $d \geq 2$ and establish Lemma 3.3.

Proof of Lemma 3.3. By Lemma 2.1, we can assume without loss of generality that $s_{j}$ is positioned on $(-x, 0, \ldots, 0)$ (generality is maintained even though we fixed $q$ to be on the origin). For $p(\Theta) \in \mathrm{bd}(\mathrm{B})$, it holds that $\operatorname{dist}\left(s_{j}, p(\Theta)\right)^{2}=x^{2}+2 r \cdot x \cdot \cos \left(\theta_{1}\right)+r^{2}$. Note that $\operatorname{dist}\left(s_{j}, p(\Theta)\right)^{2}$ is a function only of the first angle $\theta_{1}$ and it is independent of the rest of the angles. Since the function $\mathrm{E}_{\mathcal{A}}\left(s_{j}, p\right)$ is uniformly continuous and bounded on the closed set $\mathrm{B}=\mathrm{B}(q, r)$, it follows that it is Riemann integrable, and therefore

$$
\begin{aligned}
\varepsilon_{j}(q, r)= & \lim _{\bar{X} \in \mathbb{N}_{\geq 1}^{d-1},\|\bar{X}\|_{1} \rightarrow \infty} \varepsilon_{j}(q, r, \bar{X}) \\
= & \lim _{\bar{X} \in \mathbb{N}_{\geq 1}^{d-1},\|\bar{X}\|_{1} \rightarrow \infty} \sum_{i_{d-1}}^{\chi_{d-1}} \ldots \sum_{j_{1}=0}^{\chi_{1}} \mathrm{E}_{\mathcal{A}}\left(s_{j}, p\left(i_{1} \cdot \Delta \theta_{1}, \ldots, i_{d-1} \cdot \Delta \theta_{d-1}\right)\right) /\left(\chi_{1} \cdot \ldots \cdot \chi_{d-1}\right) \\
= & \frac{1}{(2 \pi)^{d-1}} \cdot \lim _{\bar{X} \in \mathbb{N}_{\geq 1}^{d-1},\|\bar{X}\|_{1} \rightarrow \infty} \sum_{i_{d-1}}^{\chi_{d-1}} \ldots \sum_{i_{1}}^{\chi_{1}} \Delta \theta_{1} \cdot \ldots \cdot \Delta \theta_{d-1} \cdot \mathrm{E}_{\mathcal{A}} \\
& \times\left(s_{j}, p\left(i_{1} \cdot \Delta \theta_{1}, \ldots, i_{d-1} \cdot \Delta \theta_{d-1}\right)\right) \\
= & \frac{1}{(2 \pi)^{d-1}} \cdot \int_{\theta_{d-1}=0}^{2 \pi} \ldots \int_{\theta_{1}=0}^{2 \pi} \frac{d \theta_{1} \ldots d \theta_{d-1}}{\left(x^{2}+2 r \cdot x \cdot \cos \left(\theta_{1}\right)+r^{2}\right)} \\
= & \frac{1}{2 \pi} \int_{\theta_{1}=0}^{2 \pi} \frac{d \theta_{1}}{\left(x^{2}+2 r \cdot x \cdot \cos \left(\theta_{1}\right)+r^{2}\right)}=\frac{\psi_{j}}{\left|\operatorname{dist}\left(s_{j}, q\right)^{2}-r^{2}\right|},
\end{aligned}
$$

where the last equality follows by the proof of Claim 7.1 of [11].

\section{PROOFS OF LEMMAS OF MAIN RESULT}

\section{C.1 Proof of Lemma 3.4}

First, recall that since $\mathrm{B} \cap S=\emptyset$, the function $\operatorname{SINR}_{\mathcal{A}}\left(s_{j}, p\right)$ is continuous on B. For proving Part (a), note that $\operatorname{MIN}\left(\mathcal{A}, s_{0}\right) \leq \operatorname{AVG}\left(\mathcal{A}, s_{0}\right)$ (this always holds for the discrete case, and since the function $\mathrm{E}_{\mathcal{A}}\left(s_{j}, p\right)$ is strictly positive, it holds for the continuous case as well). Assume, towards contradiction, that $\operatorname{MIN}\left(\mathcal{A}, s_{0}\right)=\operatorname{AVG}\left(\mathcal{A}, s_{0}\right)$ and that there exists a point $p \in \operatorname{bd}(\mathrm{B})$ such that $\operatorname{SINR}_{\mathcal{A}}\left(s_{0}, p\right) \neq \operatorname{MIN}\left(\mathcal{A}, s_{0}\right)$. Hence necessarily $\operatorname{SINR}_{\mathcal{A}}\left(s_{0}, p\right)>\operatorname{MIN}\left(\mathcal{A}, s_{0}\right)$, implying that $\operatorname{AVG}\left(\mathcal{A}, s_{0}\right)>\operatorname{MIN}\left(\mathcal{A}, s_{0}\right)$, contradiction. Part (b) follows by definition as $p_{L}, p_{R} \in \mathrm{bd}(\mathrm{B})$.

\section{C.2 Proof of Lemma 3.5}

Let $\alpha^{\prime}=\alpha / 2$. For ease of analysis, we consider the two dimensional case but the proof naturally extends to any $d \geq 2$. For simplicity, consider the collinear rotation network $\mathcal{A}^{\prime}=$ $C R(\mathcal{A})$ For $i \in\{0, \ldots, n\})$, let $\theta_{i}$ be the angle of $s_{i}$ with respect to the origin $q$, i.e., so that $s_{i}=\left(\sqrt{\rho_{i}} \cos \theta_{i}, \sqrt{\rho_{i}} \sin \theta_{i}\right)$. By the cosine theorem, applied to the triangle defined by the points 
$(0,0),(1,0)$ and $s_{i}$,

$$
\operatorname{dist}\left(s_{i}, p_{L}\right)^{2}=\rho_{i}+2 \sqrt{\rho_{i}} \cos \theta_{i}+1 \text { and } \operatorname{dist}\left(s_{i}, p_{R}\right)^{2}=\rho_{i}-2 \sqrt{\rho_{i}} \cos \theta_{i}+1,
$$

and thus

$$
\operatorname{dist}\left(s_{i}, p_{L}\right)^{2}+\operatorname{dist}\left(s_{i}, p_{R}\right)^{2}=2\left(\rho_{i}+1\right) .
$$

For $i=0, \ldots, n$, let $x_{i}=\frac{\operatorname{dist}\left(s_{i}, p_{L}\right)^{2}}{2\left(\rho_{i}+1\right)}$, so $x_{i} \in(0,1)$, and $1-x_{i}=\frac{\operatorname{dist}\left(s_{i}, p_{R}\right)^{2}}{2\left(\rho_{i}+1\right)}$. Let $a_{i}=\frac{\psi_{i}}{\psi_{0}} \cdot\left(\frac{\rho_{0}+1}{\rho_{i}+1}\right)^{\alpha / 2}$ for $i=1, \ldots, n$. By Equation (1),

$$
\operatorname{SINR}_{\mathcal{A}}^{-1}\left(s_{0}, p_{L}\right)=\sum_{i=1}^{n} a_{i}\left(\frac{x_{0}}{x_{i}}\right)^{\alpha / 2} \text { and } \operatorname{SINR}_{\mathcal{A}}^{-1}\left(s_{0}, p_{R}\right)=\sum_{i=1}^{n} a_{i}\left(\frac{1-x_{0}}{1-x_{i}}\right)^{\alpha / 2} .
$$

Recall that the angles in the polar coordinates of the corresponding stations $s_{0}^{\prime}, \ldots, s_{n}^{\prime}$ are $\theta_{0}^{\prime}=\pi / 2$ and $\theta_{1}^{\prime}=\cdots=\theta_{n}^{\prime}=3 \pi / 2$, hence $\operatorname{dist}\left(s_{i}^{\prime}, p_{j}\right)=\sqrt{\rho_{i}+1}$ for $j=1,2$, and therefore $\operatorname{SINR}_{\mathcal{H}^{\prime}}^{-1}\left(s_{0}^{\prime}, p_{L}\right)=$ $\operatorname{SINR}_{\mathcal{A}^{\prime}}^{-1}\left(s_{0}^{\prime}, p_{R}\right)=\sum_{i=1}^{n} a_{i}$.

Applying Proposition A.1 with $x=x_{0}$ and $y_{i}=x_{i}$ for $i=1, \ldots, n$, we get that

$$
\max \left\{\sum_{i=1}^{n} a_{i}\left(\frac{x_{0}}{x_{i}}\right)^{\alpha}, \sum_{i=1}^{n} a_{i}\left(\frac{1-x_{0}}{1-x_{i}}\right)^{\alpha}\right\} \geq \sum_{i=1}^{n} a_{i}
$$

for all $x_{0}, \ldots, x_{n} \in(0,1)$. This, in turn, implies that

$$
\max \left\{\operatorname{SINR}_{\mathcal{A}}^{-1}\left(s_{0}, p_{L}\right), \operatorname{SINR}_{\mathcal{A}}^{-1}\left(s_{0}, p_{R}\right)\right\} \geq \operatorname{SINR}_{\mathcal{F}^{\prime}}^{-1}\left(s_{0}^{\prime}, p_{L}\right)=\operatorname{SINR}_{\mathcal{H}^{\prime}}^{-1}\left(s_{0}^{\prime}, p_{R}\right)=\sum_{i=1}^{n} a_{i} .
$$

The lemma follows.

\section{C.3 Proof of Lemma 3.6}

Consider an arbitrary ball $\widetilde{B}$ satisfying $\widetilde{B} \cap S=\emptyset$. Define $\min (\widetilde{B})=\min \left\{\operatorname{SINR}_{\mathcal{A}}\left(s_{0}, p\right) \mid p \in \widetilde{B}\right\}$. The strong minimum principle implies that every point $p \in \widetilde{B}$ such that $\operatorname{SINR}_{\mathcal{A}}\left(s_{0}, p\right)=\min (\widetilde{B})$ must satisfy $p \in \operatorname{bd}(\widetilde{B})$. Assume towards contradiction that there exists an interior point $\check{p}$ in $\widetilde{B} \backslash \operatorname{bd}(\widetilde{B})$ that achieves the minimum, i.e., such that $\operatorname{SINR}_{\mathcal{A}}\left(s_{0}, \check{p}\right)=\min (\widetilde{B})$. Let $\check{r}$ be a sufficiently small radius for which $B(\check{p}, \check{r}) \subset \widetilde{B}$ is an internal ball centered at $\check{p}$ (such $\check{r}$ exists since $\check{p}$ is internal in $\widetilde{B}$.) For every point $p$ in $B(\check{p}, \check{r} / 2)$, let

$$
\epsilon(p)=\operatorname{SINR}_{\mathcal{A}}\left(s_{0}, p\right)-\min \left\{\operatorname{SINR}_{\mathcal{A}}\left(s_{0}, w\right) \mid w \in \operatorname{bd}(B(p, \check{r} / 2))\right\} .
$$

Note that by the minimality of $\check{p}$ in $\widetilde{B}, \epsilon(\check{p}) \leq 0$.

We now consider two cases. If $\check{p}$ is not not equidistant from all stations, $s_{0}, \ldots, s_{n}$, then by applying Equation (3) for the ball $B(\check{p}, \check{r} / 2)$ (which is station-free since it is contained in the station-free ball $\widetilde{B}$ ), we get that $\epsilon(\check{p})>0$, leading to contradiction.

Else, if $\check{p}$ is equidistant from all stations $s_{0}, \ldots, s_{n}$, then this is the only such point. Consequently, for any $p \in B(\check{p}, \check{r} / 2) \backslash\{\check{p}\}$, by applying Equation (3) for the (station-free) ball $B(p, \check{r} / 2)$, we get that $\epsilon(p)>0$. Since the function $\epsilon(\cdot)$ is continuous in $B(\check{p}, \check{r} / 2)$, it must be that $\epsilon(\check{p})=0$. This, in turn, implies the existence of some $w \in \operatorname{bd}(B(\check{p}, \check{r} / 2))$ with the same SINR value as $\check{p}$, i.e., $\operatorname{SINR}_{\mathcal{A}}\left(s_{0}, w\right)=\operatorname{SINR}_{\mathcal{A}}\left(s_{0}, \check{p}\right)$. Since $w \neq \check{p}$, it is not equidistant from the stations, so one can apply Equation (3) for the ball $B(w, \check{r} / 2)$, which implies the existence of a point $w^{\prime} \in \operatorname{bd}(B(w, \check{r} / 2)) \subset \widetilde{B}$ with $\operatorname{SINR}_{\mathcal{A}}\left(s_{0}, w^{\prime}\right)<\operatorname{SINR}_{\mathcal{A}}\left(s_{0}, w\right)=\operatorname{SINR}_{\mathcal{A}}\left(s_{0}, \check{p}\right)$, in contradiction to the minimality of $\check{p}$. The claim follows. 


\section{C.4 Proof of Lemma 3.8}

We make use of the following lower bounds on $\operatorname{MAX}\left(\mathcal{A}, s_{0}, p_{L}, p_{R}\right)$ and an exact expression for $\mathrm{AVG}^{-1}\left(\mathcal{A}, s_{0}\right)$ as a function of the $s_{i}-q$ distances $\rho_{0}, \ldots, \rho_{n}$ and the transmission powers $\psi_{0}, \ldots, \psi_{n}$.

Claim C.1. (a) If the network $\mathcal{A}$ is $y$-collinear then $\operatorname{MAX}\left(\mathcal{A}, s_{0}, p_{L}, p_{R}\right)=\operatorname{SUM}\left(\mathcal{A}, s_{0}\right)$.

(b) In all other cases, $\operatorname{MAX}\left(\mathcal{A}, s_{0}, p_{L}, p_{R}\right)>\operatorname{SUM}\left(\mathcal{A}, s_{0}\right)$,

(c) $\operatorname{AVG}^{-1}\left(\mathcal{A}, s_{0}\right)=\sum_{i=1}^{n} \frac{\psi_{i} \cdot\left(\rho_{0}-1\right)}{\psi_{0} \cdot\left(\rho_{i}-1\right)}+\frac{N \cdot\left(\rho_{0}-1\right)}{\psi_{0}}$.

Proof. Note that all interfering stations are on the $y$-axis iff the distances of the interfering stations from $p_{L}$ and $p_{R}$ are the same (i.e., $\operatorname{dist}\left(s_{i}, p_{L}\right)=\operatorname{dist}\left(s_{i}, p_{R}\right)$ for every $\left.s_{i} \in S\right)$. By the proof of Lemma 3.5 it then holds that Proposition A.1 can be applied with a strict inequality (in this case, in the proof of Lemma 3.5, $x_{i}=1 / 2$ for every $i \in\{0, \ldots, n\}$ ). Hence, the equality of Part (a) holds iff $\mathcal{A}$ is $y$-collinear and Part (b) holds with a strict inequality for the remaining cases. For Part (c), recall that $\varepsilon\left(s_{i}, \mathrm{~B}\right)$ is the average strength of $s_{i}$ experienced at bd(B). By Lemma 3.3,

$$
\varepsilon\left(s_{i}, \mathrm{~B}\right)=\frac{\psi_{i}}{\left|\rho_{i}-r^{2}\right|}=\frac{\psi_{i}}{\rho_{i}-1},
$$

where the last equality follows by the fact that all stations $s_{i}$ are outside the ball B and hence $\rho_{i} \geq r=1$. By plugging this into Equation (2), part (c) follows.

Since $N=0$, it follows that

$$
\operatorname{SINR}_{\mathcal{A}_{0}}^{-1}\left(s_{0}, q\right)=\sum_{i=1}^{n} \frac{\psi_{i} \cdot \rho_{0}}{\psi_{0} \cdot \rho_{i}}
$$

In addition, by Claim C.1(c),

$$
\mathrm{AVG}^{-1}\left(\mathcal{A}_{0}, s_{0}\right)=\sum_{i=1}^{n} \frac{\psi_{i} \cdot\left(\rho_{0}-1\right)}{\psi_{0} \cdot\left(\rho_{i}-1\right)}
$$

and by Claim C.1(b,c),

$$
\operatorname{MAX}\left(\mathcal{A}_{0}, s_{0}, p_{L}, p_{R}\right) \geq \sum_{i=1}^{n} \frac{\psi_{i}}{\psi_{0}} \cdot\left(\frac{\rho_{0}+1}{\rho_{i}+1}\right) .
$$

Then, for the three subsets of $S$ defined in Equation (7), we have

$$
\begin{array}{ll}
\frac{\rho_{0}}{\rho_{i}}>\frac{\rho_{0}-1}{\rho_{i}-1} & \text { for every } s_{i} \in S_{\text {close }}, \\
\frac{\rho_{0}}{\rho_{i}}<\frac{\rho_{0}+1}{\rho_{i}+1} & \text { for every } s_{i} \in S_{f a r}, \\
\frac{\rho_{0}}{\rho_{i}}=\frac{\rho_{0}+1}{\rho_{i}+1}=\frac{\rho_{0}-1}{\rho_{i}-1} & \text { for every } s_{i} \in S_{e q},
\end{array}
$$

where the last equality holds since $\rho_{i}>1$ for $i=0, \ldots, n$ (as no station is in the ball B). By the definition of the center $q, S \backslash S_{e q} \neq \emptyset$, that is, not all stations are positioned at the same distance from $q$.

Following the intuition sketched earlier, we first consider two extreme cases. For a network $\mathcal{A}_{\text {far }}$ all of whose stations are far $\left(S=S_{\text {far }}\right)$, the lemma readily follows by establishing property (4). This is shown by combining inequalities (C.1), (C.3) and (C.5), which yields

$$
\operatorname{SINR}_{\mathcal{A}_{f a r}}^{-1}\left(s_{0}, q\right)=\sum_{i=1}^{n} \frac{\psi_{i} \cdot \rho_{0}}{\psi_{0} \cdot \rho_{i}}<\sum_{i=1}^{n} \frac{\psi_{i} \cdot\left(\rho_{0}+1\right)}{\psi_{0} \cdot\left(\rho_{i}+1\right)} \leq \operatorname{MAX}\left(\mathcal{A}_{f a r}, s_{0}, p_{L}, p_{R}\right) .
$$


Hence Equation (4) holds, satisfying the dual minimality condition. At the other extreme, for a network $\mathcal{A}_{\text {close }}$ all of whose stations are close $\left(S=S_{\text {close }}\right)$, we have $\sum_{i=1}^{n} \frac{\psi_{i} \cdot \rho_{0}}{\psi_{0} \cdot \rho_{i}}>\sum_{i=1}^{n} \frac{\psi_{i} \cdot\left(\rho_{0}+1\right)}{\psi_{0} \cdot\left(\rho_{i}+1\right)}$, so Inequality (C.3) cannot be applied. Instead, in this case we prove the lemma by establishing property (5). Combining together inequalities (C.1), (C.4) and (C.2), we have

$$
\operatorname{SINR}_{\mathcal{A}_{\text {close }}}^{-1}\left(s_{0}, q\right)=\sum_{i=1}^{n} \frac{\psi_{i} \cdot \rho_{0}}{\psi_{0} \cdot \rho_{i}}<\sum_{i=1}^{n} \frac{\psi_{i} \cdot\left(\rho_{0}-1\right)}{\psi_{0} \cdot\left(\rho_{i}-1\right)}=\operatorname{AVG}^{-1}\left(\mathcal{A}_{\text {close }}, s_{0}\right) \text {. }
$$

Hence Equation (5) holds, and again the dual minimality condition is satisfied.

We now turn to discuss the more general case, in which $S$ may contain both far and close stations, i.e., $S_{\text {close }} \neq \emptyset$ and $S_{\text {far }} \neq \emptyset$. Clearly, if Equation (4) happens to hold, then the lemma follows as well. Hence, hereafter assume that

$$
\operatorname{SINR}_{\mathcal{A}_{0}}^{-1}\left(s_{0}, q\right) \geq \operatorname{MAX}\left(\mathcal{A}_{0}, s_{0}, p_{L}, p_{R}\right)
$$

(as shown later, this happens when the net interference effect of the far stations $S_{\text {far }}$ on the center $q$ dominates the effect of the close stations $S_{\text {close }}$ ), and establish property (5). By Ineq. (C.1) and (C.3), Ineq. (C.6) implies that $\sum_{i=1}^{n} \frac{\psi_{i} \cdot \rho_{0}}{\psi_{0} \cdot \rho_{i}} \geq \sum_{i=1}^{n} \frac{\psi_{i} \cdot\left(\rho_{0}+1\right)}{\psi_{0} \cdot\left(\rho_{i}+1\right)}$, or, after rearranging, as

$$
\sum_{i=1}^{n} \frac{\psi_{i}}{\psi_{0}} \cdot \frac{\rho_{0}-\rho_{i}}{\rho_{i}\left(\rho_{i}+1\right)} \geq 0
$$

Partitioning the summation into separate summations on the close and far stations, and noting that $\rho_{0}-\rho_{i}=\left|\rho_{0}-\rho_{i}\right|$ for every $s_{i} \in S_{\text {close }}, \rho_{0}-\rho_{i}=0$ for every $s_{i} \in S_{e q}$, and $\rho_{0}-\rho_{i}=-\left|\rho_{0}-\rho_{i}\right|$ for every $s_{i} \in S_{\text {far }}$, we have that

$$
\sum_{s_{i} \in S_{\text {close }}}\left|\frac{\psi_{i}}{\psi_{0}} \cdot \frac{\rho_{0}-\rho_{i}}{\rho_{i}\left(\rho_{i}+1\right)}\right|-\sum_{s_{i} \in S_{\text {far }}}\left|\frac{\psi_{i}}{\psi_{0}} \cdot \frac{\rho_{0}-\rho_{i}}{\rho_{i}\left(\rho_{i}+1\right)}\right| \geq 0
$$

(Since for every station $s_{i} \in S_{e q}, \rho_{0}-\rho_{i}=0$, these stations contribute zero to the summation, so only stations in $S_{\text {far }}$ and $S_{\text {close }}$ need to be accounted for.) Note that since $\rho_{0}>1$, it follows that $\frac{\rho_{0}+1}{\rho_{0}-1}>0$, so the above inequality can be written as

$$
\frac{\rho_{0}+1}{\rho_{0}-1}\left(\sum_{s_{i} \in S_{\text {close }}}\left|\frac{\psi_{i}}{\psi_{0}} \cdot \frac{\rho_{0}-\rho_{i}}{\rho_{i}\left(\rho_{i}+1\right)}\right|-\sum_{s_{i} \in S_{\text {far }}}\left|\frac{\psi_{i}}{\psi_{0}} \cdot \frac{\rho_{0}-\rho_{i}}{\rho_{i}\left(\rho_{i}+1\right)}\right|\right) \geq 0 .
$$

Moreover, for $s_{i} \in S_{\text {close }}$, it holds that $\rho_{0}>\rho_{i}>1$, implying that $\frac{\rho_{i}+1}{\rho_{i}-1}>\frac{\rho_{0}+1}{\rho_{0}-1}$, hence

$$
\sum_{s_{i} \in S_{\text {close }}}\left|\frac{\psi_{i}}{\psi_{0}} \cdot \frac{\rho_{0}-\rho_{i}}{\rho_{i}\left(\rho_{i}-1\right)}\right|>\frac{\rho_{0}+1}{\rho_{0}-1} \cdot \sum_{s_{i} \in S_{\text {close }}}\left|\frac{\psi_{i}}{\psi_{0}} \cdot \frac{\rho_{0}-\rho_{i}}{\rho_{i}\left(\rho_{i}+1\right)}\right| .
$$

Similarly, for $s_{i} \in S_{f a r}, \rho_{i}>\rho_{0}>1$, and hence $\frac{\rho_{i}+1}{\rho_{i}-1}<\frac{\rho_{0}+1}{\rho_{0}-1}$, so

$$
\sum_{s_{i} \in S_{f a r}}\left|\frac{\psi_{i}}{\psi_{0}} \cdot \frac{\rho_{0}-\rho_{i}}{\rho_{i}\left(\rho_{i}-1\right)}\right|<\frac{\rho_{0}+1}{\rho_{0}-1} \cdot \sum_{s_{i} \in S_{f a r}}\left|\frac{\psi_{i}}{\psi_{0}} \cdot \frac{\rho_{0}-\rho_{i}}{\rho_{i}\left(\rho_{i}+1\right)}\right| .
$$

Combining Ineq. (C.8), (C.9) and (C.10), we get that

$$
\sum_{s_{i} \in S_{\text {close }}}\left|\frac{\psi_{i}}{\psi_{0}} \cdot \frac{\rho_{0}-\rho_{i}}{\rho_{i}\left(\rho_{i}-1\right)}\right|-\sum_{s_{i} \in S_{\text {far }}}\left|\frac{\psi_{i}}{\psi_{0}} \cdot \frac{\rho_{0}-\rho_{i}}{\rho_{i}\left(\rho_{i}-1\right)}\right|>0 .
$$


We now complete the proof by noting that

$$
\begin{aligned}
\operatorname{SINR}_{\mathcal{A}_{0}}^{-1}\left(s_{0}, q\right)-\operatorname{AVG}^{-1}\left(\mathcal{A}_{0}, s_{0}\right) & =\sum_{i=1}^{n} \frac{\psi_{i} \cdot \rho_{0}}{\psi_{0} \cdot \rho_{i}}-\sum_{i=1}^{n} \frac{\psi_{i} \cdot\left(\rho_{0}-1\right)}{\psi_{0} \cdot\left(\rho_{i}-1\right)}=\sum_{i=1}^{n}\left(\frac{\psi_{i}}{\psi_{0}} \cdot \frac{\rho_{i}-\rho_{0}}{\rho_{i}\left(\rho_{i}-1\right)}\right) \\
& =\sum_{s_{i} \in S_{\text {far }}}\left|\frac{\psi_{i}}{\psi_{0}} \cdot \frac{\rho_{0}-\rho_{i}}{\rho_{i}\left(\rho_{i}-1\right)}\right|-\sum_{s_{i} \in S_{\text {close }}}\left|\frac{\psi_{i}}{\psi_{0}} \cdot \frac{\rho_{0}-\rho_{i}}{\rho_{i}\left(\rho_{i}-1\right)}\right|<0,
\end{aligned}
$$

where the first equality holds by Ineq. (C.1) and Ineq. (C.2), the third equality holds since $\rho_{i} \leq \rho_{0}$ for every $s_{i} \in S_{\text {close }}$ and $\rho_{i}>\rho_{0}$ for every $s_{i} \in S_{\text {far }}$, and the last inequality holds by Ineq. (C.11). Hence Equation (5) holds, so $\mathcal{A}_{0}$ satisfies the dual minimality condition. The claim now follows by Lemma 3.7.

\section{C.5 Proof of Lemma 3.13}

First, note that the SINR function restricted to any straight line segment $\overline{p q}$ is not constant. This holds as the characteristic polynomial of the SINR function has a finite positive degree, hence its derivative cannot be zero over a segment.

Assume, towards contradiction, that there is an interior point $p \in \overline{q_{1} q_{2}} \operatorname{such}$ that $\operatorname{SINR}_{\mathcal{A}}\left(s_{0}, p\right)=$ $\operatorname{MIN}\left(\mathcal{A}, s_{0}, q_{1}, q_{2}\right)$. Without loss of generality, assume that $\operatorname{SINR}_{\mathcal{A}}\left(s_{0}, q_{1}\right) \leq \operatorname{SINR}_{\mathcal{A}}\left(s_{0}, q_{2}\right)$. We consider two cases.

Case (a): $\operatorname{SINR}_{\mathcal{A}}\left(s_{0}, q_{1}\right)<\operatorname{SINR}_{\mathcal{A}}\left(s_{0}, q_{2}\right)$. Since the $\operatorname{SINR}$ function is not constant in the segment $\overline{q_{1} p}$, there exists some point $w_{1} \in \overline{q_{1} p}$ such that $\operatorname{SINR}_{\mathcal{A}}\left(s_{0}, w_{1}\right) \neq \operatorname{SINR}_{\mathcal{A}}\left(s_{0}, q_{1}\right)$ and by Corollary 3.12, necessarily, $\operatorname{SINR}_{\mathcal{A}}\left(s_{0}, w_{1}\right)>\operatorname{SINR}_{\mathcal{A}}\left(s_{0}, q_{1}\right)$. Since $p$ is an interior point in the segment $\overline{w_{1} q_{2}}$ but $\operatorname{SINR}_{\mathcal{A}}\left(s_{0}, p\right)<\operatorname{SINR}_{\mathcal{A}}\left(s_{0}, w_{1}\right), \operatorname{SINR}_{\mathcal{A}}\left(s_{0}, q_{2}\right)$, we end with a contradiction to Corollary 3.12 .

Case (b): $\operatorname{SINR}_{\mathcal{A}}\left(s_{0}, q_{1}\right)=\operatorname{SINR}_{\mathcal{A}}\left(s_{0}, q_{2}\right)$. Since the SINR function is not constant in the segments $\overline{q_{1} p}$ and $\overline{p q_{2}}$, there exists an interior point $w_{1}$ (resp., $w_{2}$ ) in the segment $\overline{q_{1} p}$ (resp., $\overline{p q_{2}}$ ) such that

$$
\operatorname{SINR}_{\mathcal{A}}\left(s_{0}, w_{1}\right), \operatorname{SINR}_{\mathcal{A}}\left(s_{0}, w_{2}\right) \neq \operatorname{SiNR}_{\mathcal{A}}\left(s_{0}, p\right) .
$$

Combining this with Corollary 3.12 for the points $w_{1}, w_{2} \in \overline{q_{1} q_{2}}$, it holds that

$$
\operatorname{SINR}_{\mathcal{A}}\left(s_{0}, w_{1}\right), \operatorname{SINR}_{\mathcal{A}}\left(s_{0}, w_{2}\right)>\operatorname{SINR}_{\mathcal{A}}\left(s_{0}, p\right) .
$$

But this is in contradiction with the fact that $\operatorname{SINR}_{\mathcal{A}}\left(s_{0}, p\right)>\operatorname{MIN}\left(\mathcal{A}, s_{0}, w_{1}, w_{2}\right)$ (which follows by applying Corollary 3.12 for the segment $\overline{w_{1} w_{2}}$, since $\left.p \in \overline{w_{1} w_{2}}\right)$. The lemma follows.

\section{C.6 Proof of Lemma 3.15}

We first state and prove the following two claims.

ClaIm C.2. If Equation (11) holds, then Equation (12) holds as well, i.e., $\operatorname{SINR}_{\mathcal{A}}^{-1}\left(s_{0}, q\right)=$ $\operatorname{AVG}^{-1}\left(\mathcal{A}, s_{0}\right)$.

Proof. It is sufficient to show that if $\operatorname{SINR}_{\mathcal{A}}^{-1}\left(s_{0}, q\right)>\operatorname{AVG}^{-1}\left(\mathcal{A}, s_{0}\right)$, then $\operatorname{SINR}_{\mathcal{A}}^{-1}\left(s_{0}, q\right)<$ $\operatorname{MAX}\left(\mathcal{A}, s_{0}, p_{L}, p_{R}\right)$. To show this, assume towards contradiction that

$(\mathrm{CON} 1) \operatorname{SINR}_{\mathcal{A}}^{-1}\left(s_{0}, q\right)>\operatorname{AVG}^{-1}\left(\mathcal{A}, s_{0}\right)$; and

$(\mathrm{CON} 2) \operatorname{SINR}_{\mathcal{A}}^{-1}\left(s_{0}, q\right) \geq \operatorname{MAX}\left(\mathcal{A}, s_{0}, p_{L}, p_{R}\right)$.

Let $\ell^{*}$ be a sufficiently large real number such that $\mathcal{A}\left(\ell^{*}\right) \in \mathcal{N} \mathcal{F}$ and

$$
\operatorname{SINR}_{\mathcal{A}}^{-1}\left(s_{0}, q\right)>\operatorname{AVG}^{-1}\left(\mathcal{A}\left(\ell^{*}\right), s_{0}\right) \text {. }
$$

(By Property (P2) of Lemma 3.9, $\lim _{\ell \rightarrow \infty} \mathrm{AVG}^{-1}\left(\mathcal{A}(\ell), s_{0}\right)=\mathrm{AVG}^{-1}\left(\mathcal{A}, s_{0}\right)$, hence such a real $\ell^{*}$ exists by the contradictory assumption (CON1).) 
By the contradictory assumption $(\mathrm{CON} 2), \operatorname{SINR}_{\mathcal{A}}^{-1}\left(s_{0}, q\right) \geq \operatorname{MAX}\left(\mathcal{A}, s_{0}, p_{L}, p_{R}\right)$. Combining this with Property (P4) of Lemma 3.9, we have

$$
\operatorname{SINR}_{\mathcal{A}}^{-1}\left(s_{0}, q\right)>\operatorname{MAX}\left(\mathcal{A}\left(\ell^{*}\right), s_{0}, p_{L}, p_{R}\right)
$$

Finally, by Property (P1) of Lemma 3.9, $\operatorname{SINR}_{\mathcal{A}\left(\ell^{*}\right)}^{-1}\left(s_{0}, q\right)=\operatorname{SINR}_{\mathcal{A}}^{-1}\left(s_{0}, q\right)$. Combining this with Equation (C.12) and (C.13), we have that

$$
\operatorname{SINR}_{\mathcal{A}\left(\ell^{*}\right)}^{-1}\left(s_{0}, q\right)>\max \left\{\operatorname{MAX}\left(\mathcal{A}\left(\ell^{*}\right), s_{0}, p_{L}, p_{R}\right), \mathrm{AVG}^{-1}\left(\mathcal{A}\left(\ell^{*}\right), s_{0}\right)\right\},
$$

which contradicts Lemma 3.8 since $\mathcal{A}\left(\ell^{*}\right)$ is a noise-free network. Claim C.2 follows.

CLAIM C.3. If $\mathcal{A}$ is a non-collinear network satisfying Equation (11), then

$$
\operatorname{AVG}^{-1}\left(\mathcal{A}, s_{0}\right)>\operatorname{MAX}\left(\mathcal{A}, s_{0}, p_{L}, p_{R}\right) .
$$

Proof. By Equation (12), established in Claim C.2, it suffices to show that $\operatorname{AVG}^{-1}\left(\mathcal{A}, s_{0}\right) \neq$ $\operatorname{MAX}\left(\mathcal{A}, s_{0}, p_{L}, p_{R}\right)$. Assume, towards contradiction, that $\mathrm{AVG}^{-1}\left(\mathcal{A}, s_{0}\right)=\operatorname{MAX}\left(\mathcal{A}, s_{0}, p_{L}, p_{R}\right)$. Then by Claim C.2,

$$
\operatorname{SINR}_{\mathcal{A}}^{-1}\left(s_{0}, q\right)=\operatorname{AVG}^{-1}\left(\mathcal{A}, s_{0}\right)=\operatorname{MAX}\left(\mathcal{A}, s_{0}, p_{L}, p_{R}\right) .
$$

We next construct two networks $\mathcal{A}^{\prime}$ and $\mathcal{A}^{\prime \prime}$, with stations $s_{0}^{\prime}$ and $s_{0}^{\prime \prime}$, respectively, such that

(Q1) $\operatorname{SINR}_{\mathcal{A}}^{-1}\left(s_{0}, q\right)=\operatorname{SINR}_{\mathcal{A}^{\prime}}^{-1}\left(s_{0}^{\prime}, q\right)=\operatorname{SINR}_{\mathcal{H}^{\prime \prime}}^{-1}\left(s_{0}^{\prime \prime}, q\right)$;

(Q2) $\operatorname{AVG}^{-1}\left(\mathcal{A}^{\prime \prime}, s_{0}\right)<\operatorname{AVG}^{-1}\left(\mathcal{A}^{\prime}, s_{0}^{\prime}\right)=\operatorname{AVG}^{-1}\left(\mathcal{A}, s_{0}\right)$;

(Q3) $\operatorname{MAX}\left(\mathcal{A}^{\prime}, s_{0}^{\prime}, p_{L}, p_{R}\right), \operatorname{MAX}\left(\mathcal{A}^{\prime \prime}, s_{0}^{\prime \prime}, p_{L}, p_{R}\right)<\operatorname{MAX}\left(\mathcal{A}, s_{0}, p_{L}, p_{R}\right)$.

Note that the existence of such networks results in a contradiction. Specifically, combining Equation (C.14) with properties (Q1) and (Q2) yields that $\mathrm{AVG}^{-1}\left(\mathcal{A}^{\prime \prime}, s_{0}^{\prime \prime}\right)<\operatorname{SINR}_{\mathcal{A}^{\prime \prime}}^{-1}\left(s_{0}^{\prime \prime}, q\right)$, and by combining properties (Q1) and (Q3), $\operatorname{MAX}\left(\mathcal{A}^{\prime \prime}, s_{0}^{\prime \prime}, p_{L}, p_{R}\right)<\operatorname{SINR}_{\mathcal{A}^{\prime \prime}}^{-1}\left(s_{0}^{\prime \prime}, q\right)$, contradicting the weak minimum principle of Lemma 3.10, which implies Claim C.3.

It remains to describe the construction of the networks $\mathcal{A}^{\prime}$ and $\mathcal{A}^{\prime \prime}$. This first network, $\mathcal{A}^{\prime}=$ $\left\langle S^{\prime}=\left\{s_{0}^{\prime}, \ldots, s_{n}^{\prime}\right\}, \bar{\psi}, N, \beta, \alpha\right\rangle$, is $\mathcal{A}^{\prime}=C R(\mathcal{A})$, the collinear rotation of $\mathcal{A}$. That is, the station $s_{0}$ is located at $\left(0, \sqrt{\rho_{0}}\right)$, each other station $s_{i}^{\prime}$ (for $\left.i \in\{1, \ldots, n\}\right)$ is located at $\left(0,-\sqrt{\rho_{i}}\right)$, and the transmission power of each station $s_{i}^{\prime}$ (for $i \in\{0, \ldots, n\}$ ) is $\psi_{i}$, as illustrated in Figure 5 . Recall that all stations preserve their transmission powers as well as their distances to the ball center $q$, hence

$$
\operatorname{SINR}_{\mathcal{A}}^{-1}\left(s_{0}, q\right)=\operatorname{SINR}_{\mathcal{H}^{\prime}}^{-1}\left(s_{0}^{\prime}, q\right)=\sum_{i=1}^{n} \frac{\psi_{i} \cdot \rho_{0}}{\psi_{0} \cdot \rho_{i}}+\frac{N \cdot \rho_{0}}{\psi_{0}},
$$

implying property $(\mathrm{Q} 1)$ for the network $\mathcal{A}^{\prime}$. Since the average strength $\mathrm{AVG}^{-1}\left(\mathcal{A}, s_{0}\right)$ is a function of the distances $\rho_{i}$, it also holds that

$$
\operatorname{AVG}^{-1}\left(\mathcal{A}, s_{0}\right)=\sum_{i=1}^{n} \frac{\psi_{i} \cdot\left(\rho_{0}-1\right)}{\psi_{0} \cdot\left(\rho_{i}-1\right)}+\frac{N\left(\rho_{0}-1\right)}{\psi_{0}}=\operatorname{AVG}^{-1}\left(\mathcal{A}^{\prime}, s_{0}^{\prime}\right),
$$

implying property (Q2) for $\mathcal{A}^{\prime}$. Furthermore, since $\mathcal{A}^{\prime}$ is $y$-collinear and $\mathcal{A}$ is not, by Claim C.1(b,c) we have

$$
\operatorname{MAX}\left(\mathcal{A}^{\prime}, s_{0}^{\prime}, p_{L}, p_{R}\right)=\operatorname{SUM}\left(\mathcal{A}, s_{0}\right)<\operatorname{MAX}\left(\mathcal{A}, s_{0}, p_{L}, p_{R}\right),
$$

implying property $(\mathrm{Q} 3)$ for $\mathcal{A}^{\prime}$. Hence $\mathcal{A}^{\prime}$ satisfies its desired properties. We now turn to construct the second network $\mathcal{A}^{\prime \prime}$, which can be obtained from $\mathcal{A}^{\prime}$ in the following manner. Fix a sufficiently small positive real $\epsilon \in\left(0, \rho_{0}-1\right)$ and use $\mathcal{A}^{\prime}$ to construct an $(n+1)$ - station network $\mathcal{A}^{\prime \prime}=\left\langle S^{\prime \prime}=\right.$ $\left.\left\{s_{0}^{\prime \prime}, s_{1}^{\prime}, \ldots, s_{n}^{\prime}\right\}, \bar{\psi}^{\prime \prime}=\left(\psi_{0}^{\prime \prime}, \psi_{1}, \ldots, \psi_{n}\right), N, \beta, \alpha\right\rangle$ by substituting station $s_{0}^{\prime}$ with station $s_{0}^{\prime \prime}$ preserving the strength at $q$ (i.e., $\left.\mathrm{E}_{\mathcal{A}^{\prime \prime}}\left(s_{0}^{\prime \prime}, p\right)=\mathrm{E}_{\mathcal{H}^{\prime}}\left(s_{0}^{\prime}, q\right)\right)$. The station $s_{0}^{\prime \prime}$ is still located on the positive $y$-axis 


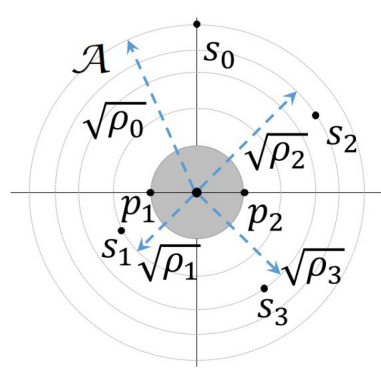

(a)

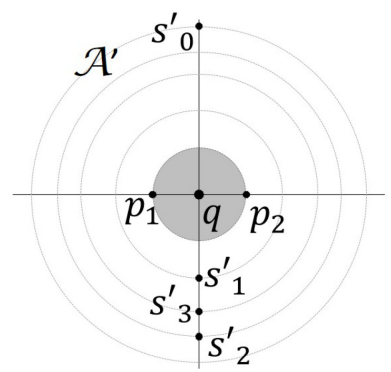

(b)

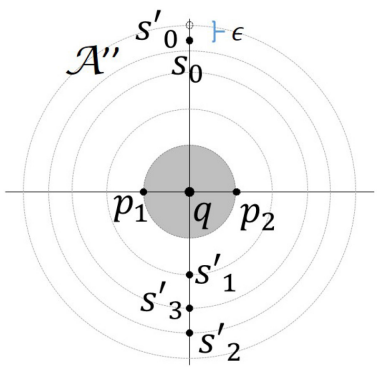

(c)

Fig. 5. (a) The original wireless network $\mathcal{A}$. (b) $\mathcal{A}^{\prime}$ obtained from $\mathcal{A}$. (c) $\mathcal{A}^{\prime \prime}$ obtained from $\mathcal{A}^{\prime}$.

but it is a bit closer to $q$ than $s_{0}^{\prime}$. That is, $s_{i}^{\prime \prime}$ is located at $\left(0, \sqrt{\rho_{0}^{\prime \prime}}\right)$, where $\rho_{0}^{\prime \prime}=\rho_{0}-\epsilon$, and its transmission power is $\psi_{0}^{\prime \prime}=\psi_{0}\left(\rho_{0}-\epsilon\right) / \rho_{0}$. Hence $\mathrm{E}_{\mathcal{A}^{\prime \prime}}\left(s_{0}^{\prime \prime}, q\right)=\frac{\psi_{0}^{\prime \prime}}{\rho_{0}-\epsilon}=\frac{\psi_{0}}{\rho_{0}}=\mathrm{E}_{\mathcal{H}^{\prime}}\left(s_{0}^{\prime}, q\right)$, and thus

$$
\operatorname{SINR}_{\mathcal{A}}\left(s_{0}, q\right)=\operatorname{SINR}_{\mathcal{H}^{\prime}}\left(s_{0}^{\prime}, q\right)=\operatorname{SINR}_{\mathcal{A}^{\prime \prime}}\left(s_{0}^{\prime \prime}, q\right),
$$

implying property $(\mathrm{Q} 1)$ for $\mathcal{A}^{\prime \prime}$. Note that $\frac{\rho_{0}^{\prime \prime}-1}{\psi_{0}^{\prime \prime}}<\frac{\rho_{0}-1}{\psi_{0}}$, hence by Claim C.1(c), for every $\epsilon \epsilon$ $(0, \rho-1)$,

$$
\operatorname{AVG}^{-1}\left(\mathcal{A}^{\prime \prime}, s_{0}^{\prime \prime}\right)=\frac{\rho_{0}^{\prime \prime}-1}{\psi_{0}^{\prime \prime}} \cdot\left(\sum_{i=1}^{n} \frac{\psi_{i}}{\rho_{i}-1}+N\right)<\frac{\rho_{0}-1}{\psi_{0}} \cdot\left(\sum_{i=1}^{n} \frac{\psi_{i}}{\rho_{i}-1}+N\right)=\operatorname{AVG}^{-1}\left(\mathcal{A}^{\prime}, s_{0}^{\prime}\right),
$$

where the last equality follows by Equation (C.16). This implies property (Q2) for $\mathcal{A}^{\prime \prime}$. It remains to show that $\mathcal{A}^{\prime \prime}$ satisfies property (Q3), namely, that $\operatorname{MAX}\left(\mathcal{A}^{\prime \prime}, s_{0}^{\prime \prime}, p_{L}, p_{R}\right)<\operatorname{MAX}\left(\mathcal{A}, s_{0}, p_{L}, p_{R}\right)$.

Since $\operatorname{SINR}_{\mathcal{A}^{\prime \prime}}^{-1}\left(s_{0}^{\prime \prime}, p_{i}\right)$ is continuous in $\epsilon$, it holds that $\lim _{\epsilon \rightarrow 0} \operatorname{SINR}_{\mathcal{A}^{\prime \prime}}^{-1}\left(s_{0}^{\prime \prime}, p_{i}\right)=\operatorname{SINR}_{\mathcal{A}^{\prime}}^{-1}\left(s_{0}^{\prime}, p_{i}\right)$ for $i \in\{L, R\}$. In addition, by Equation (C.14) and properties (Q1) and (Q3) for the network $\mathcal{A}^{\prime}$,

$$
\operatorname{MAX}\left(\mathcal{A}^{\prime}, s_{0}^{\prime}, p_{L}, p_{R}\right)<\operatorname{SINR}_{\mathcal{H}^{\prime}}^{-1}\left(s_{0}^{\prime}, p_{i}\right),
$$

and by property $(\mathrm{Q} 1), \operatorname{MAX}\left(\mathcal{A}^{\prime}, s_{0}^{\prime}, p_{L}, p_{R}\right)<\operatorname{SINR}_{\mathcal{A}^{\prime \prime}}^{-1}\left(s_{0}^{\prime \prime}, p_{i}\right)$. Since all these functions are continuous in $\epsilon$, there exists a sufficiently small $\epsilon>0$ satisfying

$$
\operatorname{MAX}\left(\mathcal{A}^{\prime \prime}, s_{0}^{\prime \prime}, p_{L}, p_{R}\right)<\operatorname{SINR}_{\mathcal{A}^{\prime \prime}}^{-1}\left(s_{0}^{\prime \prime}, q\right)
$$

Combining this with property (Q1) and Equation (C.14), we get that

$$
\operatorname{SINR}_{\mathcal{A}^{\prime \prime}}^{-1}\left(s_{0}^{\prime \prime}, q\right)=\operatorname{SINR}_{\mathcal{A}}^{-1}\left(s_{0}, q\right)=\operatorname{MAX}\left(\mathcal{A}, s_{0}, p_{L}, p_{R}\right),
$$

establishing property (Q3) for $\mathcal{A}^{\prime \prime}$. Claim C.3 follows.

We can now complete the proof of Lemma 3.15. As mentioned before, if $\mathcal{A}$ satisfies the strong dual minimality condition, then we are done by Lemma 3.7. Otherwise, $\mathcal{A}$ satisfies Equation (11), so by Claim C.3, $\operatorname{AVG}^{-1}\left(\mathcal{A}, s_{0}\right) \neq \operatorname{MAX}\left(\mathcal{A}, s_{0}, p_{L}, p_{R}\right)$. By Lemma 3.4(a), necessarily $\operatorname{AVG}^{-1}\left(\mathcal{A}, s_{0}\right)<\operatorname{MAX}\left(\mathcal{A}, s_{0}\right)$. Hence by Claim C.2, also $\operatorname{SINR}_{\mathcal{A}}^{-1}\left(s_{0}, q\right)<\operatorname{MAX}\left(\mathcal{A}, s_{0}\right)$, or equivalently, $\operatorname{SINR}_{\mathcal{A}}\left(s_{0}, q\right)>\operatorname{MIN}\left(\mathcal{A}, s_{0}\right)$, i.e., $\mathcal{A}$ satisfies the ball center condition. The lemma now follows by Lemma 3.6. 


\section{IMPROVED $\epsilon$-APPROXIMATE RECEPTION TESTING FOR ANY SHAPE $(\beta>(1+\epsilon) /(1-\epsilon))$}

Theorem D.1. For every reception threshold $\beta$, and $\epsilon \leq 1-1 / \beta$, network $\mathcal{A}=\langle d=2, S, \psi, N, \beta\rangle$ $(1+\epsilon) /(1-\epsilon), \alpha=2\rangle$, and a target station $s_{i}$, there exists an $\epsilon$-approximate testing procedure ApproxRecepTest that given a closed shape $C \subseteq \mathbb{R}^{2}$ free of stations $s_{j} \neq s_{i}$, returns in $\widetilde{O}\left(n / \epsilon^{6}+\right.$ $\left.|\operatorname{bd}(C)| /\left(\widetilde{\delta} \cdot \epsilon^{3 / 2}\right)\right)$ time a binary answer $b_{R}$ such that if $b_{R}=1$ then $C$ is $\epsilon$-close, and if $b_{R}=0$ then $C$ is non-receptive.

Our algorithm employs the approximate batched point location scheme of [2].

Theorem D.2 (Restatement of Theorem 3.6 of [2]). For a positive $\epsilon \leq 1-1 / \beta$, any fixed positive even integer $\alpha$, given a set $\mathcal{S}$ of $n$ transmitters and a set $Q$ of $m$ receivers, we can do the following in total time and space of $\widetilde{O}\left(n / \epsilon^{6}+m / \sqrt{\epsilon}\right)$ : For each $q \in Q$, we find a transmitter $s_{q}$ and compute a value $\widetilde{E}(q)$ satisfying that $\widetilde{E}(q) \in\left[(1-\epsilon) \operatorname{SINR}_{\mathcal{A}}\left(s_{q}, q\right),(1+\epsilon) \operatorname{SINR}_{\mathcal{A}}\left(s_{q}, q\right)\right]$.

We now describe the approximate testing procedure.

Procedure ApproxRecepTest $\left(\mathcal{A}, s_{i}, C, \epsilon\right)$.

(1) Sample a set of $n_{\epsilon}$ points $P$ evenly spaced on $\operatorname{bd}(C)$, where $n_{\epsilon}=\left\lceil|\mathrm{bd}(C)| / d_{\epsilon}\right\rceil$.

(2) Apply Theorem D.2 with stations $S$ of $\mathcal{A}$ and return 1 if $s_{p}=s_{i}$ and $\widetilde{E}(p) \geq(1-\epsilon) \beta$ for every $p \in P$. Otherwise, return 0 .

We now prove the correctness of our scheme.

Lemma D.3. (a) If ApproxRecepTest $\left(\mathcal{A}, s_{i}, C, \epsilon\right)$ outputs 1 , then $C$ is $\epsilon$-close to being receptive.

(b) If ApproxRecepTest $\left(\mathcal{A}, s_{i}, C, \epsilon\right)$ outputs 0 , then $C$ is non-receptive.

Proof. Suppose ApproxRecepTest $\left(\mathcal{A}, s_{i}, C, \epsilon\right)=1$. Let $P \subseteq \operatorname{bd}(C)$ be the collection of points tested by Procedure ApproxRecepTest. Since ApproxRecepTest $\left(\mathcal{A}, s_{i}, C, \epsilon\right)$ outputs 1 , it follows that for every $p \in P, \operatorname{SINR}_{\mathcal{A}}\left(s_{i}, p\right) \geq((1-\epsilon) /(1+\epsilon)) \cdot \beta$ and since $\beta>(1+\epsilon) /(1-\epsilon)$, we have that $\operatorname{SINR}_{\mathcal{A}}\left(s_{i}, p\right)>1$. By the definition of $\widetilde{\delta}, p \notin \bigcup_{j \neq i} B\left(s_{j}, \widetilde{\delta}\right)$. (Since $\widetilde{\delta}$ is decreasing as a function of $\beta$, the statement holds when using a small value of $\beta$, namely, $\beta^{\prime}=((1-\epsilon) /(1+\epsilon)) \cdot \beta$.) In addition, for every unsampled boundary point $q \in \operatorname{bd}(C) \backslash P$, it holds that there exists a sufficiently close sampled point $p \in B\left(q, d_{\epsilon}\right) \cap P$. If $p$ is in $B\left(s_{i}, \widetilde{\delta} / 2\right)$, then $q \in B\left(s_{i}, \widetilde{\delta}\right)$, and by using Lemma 4.3 with $\beta^{\prime}=((1-\epsilon) /(1+\epsilon)) \beta$, we have that $\operatorname{SINR}\left(s_{i}, q\right) \geq \beta^{\prime}$ (since every point in the ball of radius $\widetilde{\delta}$ around $s_{i}$ has SINR at least $\left.\beta^{\prime}\right)$. Else, we have that $q \notin \bigcup_{s_{j} \in S} B\left(s_{j}, \widetilde{\delta} / 2\right)$ and Observation 4.2 can be safely applied, ${ }^{9}$ yielding

$$
\operatorname{SINR}_{\mathcal{A}}\left(s_{i}, q\right) \geq\left(\frac{1-\epsilon}{1+\epsilon}\right)^{\alpha} \cdot \operatorname{SINR}_{\mathcal{A}}\left(s_{i}, p\right) \geq\left(\frac{1-\epsilon}{1+\epsilon}\right)^{\alpha+1} \beta=\beta_{\epsilon},
$$

where the last inequality follows by the fact that $\operatorname{SINR}_{\mathcal{A}}\left(s_{i}, p\right) \geq(1-\epsilon) /(1+\epsilon) \cdot \beta$ and by Equation (15). Since $C$ is free of interfering stations, by Corollary 3.16, it then holds that $\operatorname{SINR}_{\mathcal{A}}\left(s_{i}, q\right) \geq$ $\beta_{\epsilon}$ for every point $q \in C$. By Equation (15), $C$ is $\epsilon$-close to being receptive, establishing Part (a).

Part (b) follows immediately, since the algorithm outputs 0 (in step 2) if there exists a point $p$ on the boundary for which $\widetilde{E}(p)<(1-\epsilon) \beta$ and thus $\operatorname{SINR}_{\mathcal{A}}\left(s_{i}, q\right)<\beta$ and hence $C$ is non-receptive.

Theorem D.1 follows from Theorem D.2 and Lemma D.3.

\footnotetext{
${ }^{9}$ Note that for Observation 4.2 to hold, it is only important the ratio between $\widetilde{\delta}$ and $d_{\epsilon}$. That is, it does not use the fact that $\widetilde{\delta}$ is a bound on the radius of the smallest ball contained in a reception region.
} 


\section{ACKNOWLEDGMENTS}

We are grateful to the anonymous reviewers for their helpful suggestions and comments, which considerably simplified and improved the presentation.

\section{REFERENCES}

[1] Alfred V. Aho, John E. Hopcroft, and Jeffrey D. Ullman. 1976. The design and analysis of computer algorithms. (1976).

[2] Boris Aronov and Matthew J. Katz. 2018. Batched point location in SINR diagrams via algebraic tools. ACM Transactions on Algorithms (TALG) 14, 4 (2018), 1-29.

[3] C. Avin, Y. Emek, E. Kantor, Z. Lotker, D. Peleg, and L. Roditty. 2012. SINR diagrams: Convexity and its applications in wireless networks. F. ACM 59(4) (2012).

[4] Saugata Basu, Richard Pollack, and Marie-Françoise Coste-Roy. 2007. Algorithms in Real Algebraic Geometry. Vol. 10. Springer Science \& Business Media.

[5] S. Borbash and A. Ephremides. 2006. Wireless link scheduling with power control and SINR constraints. IEEE Tr. Inf. Theory (2006).

[6] M. Chiang, P. Hande, T. Lan, and C. W. Tan. 2008. Power control in wireless cellular networks. Foundations and Trends in Networking 2 (4) (2008), 381-533.

[7] R. L. Cruz and A. V. Santhanam. 2003. Optimal routing, link scheduling and power control in multi-hop wireless networks. In Proc. INFOCOM.

[8] H. T. Friis. 1946. A note on a simple transmission formula. In Proc. IRE. 34 (5), 254-256.

[9] M. M. Halldorsson and P. Mitra. 2011. Wireless capacity with oblivious power in general metrics. In Proc. SODA.

[10] E. Kantor, Z. Lotker, M. Parter, and D. Peleg. 2011. The topology of wireless communication. In Proc. STOC.

[11] E. Kantor, Z. Lotker, M. Parter, and D. Peleg. 2015. The topology of wireless communication. F. ACM 62 (2015), 37:1$37: 32$.

[12] E. Kantor, Z. Lotker, M. Parter, and D. Peleg. 2018. The topology of wireless communication on a line. TCS 711 (2018), 105-108.

[13] W. Kaplan. 1984. Maximum Principles in Differential Equations. Addison-Wesley.

[14] W. Kaplan. 2002. Advanced Calculus. Addison-Wesley.

[15] T. Kesselheim. 2011. A constant-factor approximation for wireless capacity maximization with power control in the SINR model. In Proc. SODA.

[16] T.-H. Lee, J.-C. Lin, and Y. T. Su. 1995. Downlink power control algorithms for cellular radio systems. IEEE Tr. Vehic. Technol 44 (1995), 89-94.

[17] T. Moscibroda and R. Wattenhofer. 2006. The complexity of connectivity in wireless networks. In Proc. INFOCOM.

[18] Franco P. Preparata and Michael I. Shamos. 1985. Computational Geometry: An Introduction. Springer-Verlag.

[19] J. T. Schwartz, M. Sharir, and J. E. Hopcroft. 1987. Planning, Geometry, and Complexity of Robot Motion. Intellect Books.

[20] J. Stewart. 2009. Multivariable Calculus: Concepts and Contexts. Cengage Learning.

[21] A. Tucker T. Gonzalez, and J. D. Herrera. 2014. Computing Handbook, Third Edition: Computer Science and Software Engineering. Hall/CRC.

[22] V. Vazirani. 2012. The notion of a rational convex program, and an algorithm for the Arrow-Debreu Nash bargaining game. F. ACM 59 (2) (2012).

[23] K. Wang, C. Chiasserini, R. Rao, and J. Proakis. 2005. A joint solution to scheduling and power control for multicasting in wireless ad hoc networks. f. Applied Signal Processing 2 (2005).

[24] J. Zander. 1992. Performance of optimum transmitter power control in cellular radiosystems. IEEE Tr. Vehic. Technol. 41 (1992), 57-62.

Received 12 November 2019; revised 10 January 2021; accepted 20 July 2021 